TRANSACTIONS OF THE

AMERICAN MATHEMATICAL SOCIETY

Volume 352, Number 1, Pages 1-54

S 0002-9947(99)02287-4

Article electronically published on March 29, 1999

\title{
FUNCTIONS FOR PARAMETRIZATION OF SOLUTIONS OF AN EQUATION IN A FREE MONOID
}

\author{
GENNADY S. MAKANIN AND TATIANA A. MAKANINA
}

$$
\begin{aligned}
& \text { ABstRACT. In this paper we introduce recursive functions } \\
& \qquad \begin{aligned}
\mathbf{F i} & \left(x_{1}, x_{2}\right)^{\lambda_{1}, \ldots, \lambda_{s}} \quad(s \geq 0), \\
& \mathbf{T h}_{\left(x_{1}, x_{2}, x_{3}\right)_{i}^{\lambda_{1}, \ldots, \lambda_{2 s}}}(i=1,2,3 ; s \geq 0), \\
& \mathbf{R o}\left(x_{1}, x_{2}, x_{3}\right)_{i}^{\mu_{1}, \ldots, \mu_{s}} \quad(i=1,2,3 ; s \geq 0)
\end{aligned}
\end{aligned}
$$

of the word variables $x_{1}, x_{2}, x_{3}$, natural number variables $\lambda_{k}$ and variables $\mu_{k}$ whose values are finite sequences of natural number variables. By means of these functions we give finite expressions for the family of solutions of the equation

$$
x_{1} x_{2} x_{3} x_{4}=\zeta\left(x_{1}, x_{2}, x_{3}\right) x_{5},
$$

where $\zeta\left(x_{1}, x_{2}, x_{3}\right)$ is an arbitrary word in the alphabet $x_{1}, x_{2}, x_{3}$, in a free monoid.

\section{INTRODUCTION}

In 1960 Lyndon [1], [2] considered equations with one unknown in a free group and proved that the family of solutions of such an equation can be represented by a finite number of parametric words. In $1967 \mathrm{Khmelevskii} \mathrm{[3]} \mathrm{considered} \mathrm{equations}$ with three unknowns in a free monoid and proved that the family of solutions of such an equation can be represented by a finite number of parametric words. For a short time after that it was believed that the solutions of all equations in a free group or a free monoid are parametrizable. However in 1971 Khmelevskii [4] pointed out that the solutions of Markov's equation $x_{1} x_{3} x_{2}=x_{2} x_{4} x_{1}$ with four unknowns in a free monoid is not parametrizable by a finite number of parametric words.

Parametrizations of solutions in the Lyndon-Khmelevskii sense (now called primitive parametrization) use variables of two kinds: word variables and natural number variables. We suppose that the idea of finite parametrization of the solutions of the equations in a free group and a free monoid can be saved if we admit an additional kind of variables, namely, variables whose values are the finite sequences of natural number variables. In this paper we intend to demonstrate the possibilities of the parametrization of the solutions of the equations in a free monoid by means of parametrizing functions of variables of the three mentioned kinds on a carefully chosen example of an equation in a free monoid. This equation is not bulky and "contains" many known difficult equations.

Received by the editors April 14, 1997.

1991 Mathematics Subject Classification. Primary 20M05; Secondary 03D40, 20F10.

(C)1999 American Mathematical Society 
We introduce recursive functions

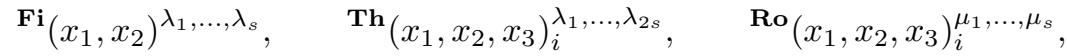

where $s \geq 0, i=1,2,3$, of the word variables $x_{1}, x_{2}, x_{3}$, natural number variables $\lambda_{k}$, and variables $\mu_{k}$ whose values are finite sequences of natural number variables. We consider here an equation of the form

$$
x_{1} x_{2} x_{3} x_{4}=\zeta\left(x_{1}, x_{2}, x_{3}\right) x_{5},
$$

where $\zeta\left(x_{1}, x_{2}, x_{3}\right)$ is an arbitrary word in the alphabet $x_{1}, x_{2}, x_{3}$ in a free monoid. We shall give concrete expression from parametric words and parametrizing functions ${ }^{\mathbf{F i}},{ }^{\mathbf{T h}}, \mathbf{R o}$, which describe the family of solutions of the equation $x_{1} x_{2} x_{3} x_{4}=$ $\zeta\left(x_{1}, x_{2}, x_{3}\right) x_{5}$ in a free monoid.

We use the preprints [5] and [6].

\section{Definitions AND NOTATION}

Let $\Pi$ be a free monoid (a free semigroup with unit) with a countable alphabet of generators

$$
a_{1}, a_{2}, \ldots, a_{k}, \ldots
$$

Let

$$
x_{1}, x_{2}, \ldots, x_{n}, \ldots
$$

be a countable alphabet of word variables.

Let

$$
\lambda_{1}, \lambda_{2}, \ldots, \lambda_{t}, \ldots
$$

be a countable alphabet of natural number variables (also called natural parameters).

Let

$$
\mu_{1}, \mu_{2}, \ldots, \mu_{u}, \ldots
$$

be a countable alphabet of variables (called second parameters) whose values are finite sequences of natural parameters.

Let

$$
\nu_{1}, \nu_{2}, \ldots, \nu_{v}, \ldots
$$

be a countable alphabet of variables whose values are finite sequences of second parameters.

Define inductively a primitive parametric word as follows: Any word on the alphabet (2) is a primitive word. If $P$ is a primitive parametric word and $\lambda$ is a natural parameter, then $(P)^{\lambda}$ is a primitive parametric word. If $P$ and $Q$ are two primitive parametric words, then $P Q$ is a primitive parametric word.

We denote by $\mathbf{L}$ the set of linear polynomials of the form $k_{0}+\sum_{i=1}^{r} k_{i} \lambda_{i}$, where $r, k_{0}, k_{1}, \ldots, k_{r}$ are natural numbers, and $\lambda_{1}, \ldots, \lambda_{r}$ are natural parameters.

A primitive parametric transformation is defined by the application

$$
\left\{\begin{array}{l}
x_{i} \rightarrow W_{i}\left(x_{1}, \ldots, x_{n}, \lambda_{1}, \ldots, \lambda_{q}\right) \quad(i=1, \ldots, n), \\
\lambda_{i} \rightarrow L_{i}\left(\lambda_{1}, \ldots, \lambda_{q}\right) \quad(i=1, \ldots, q),
\end{array}\right.
$$

where every $W_{i}$ is a primitive word, and $L_{i} \in \mathbf{L}$. The components of the form $x_{i} \rightarrow x_{i}$ and $\lambda_{i} \rightarrow \lambda_{i}$ are often omitted. 
Now we inductively define a parametric word (transformation). Any primitive parametric word (transformation) is a parametric word (transformation).

Define the function ${ }^{\mathbf{F i}}\left(x_{1}, x_{2}\right)^{\lambda_{1}, \ldots, \lambda_{s}}$ for $s \geq 0$, where $x_{1}$ and $x_{2}$ are two word variables, and $\lambda_{1}, \ldots, \lambda_{s}$ are natural parameters, inductively as follows:

$$
\begin{gathered}
\mathbf{F i}\left(x_{1}, x_{2}\right)=1, \\
{ }^{\mathbf{F i}}\left(x_{1}, x_{2}\right)^{\lambda_{1}, \ldots, \lambda_{s}}=\left({ }^{\mathbf{F i}}\left(x_{2}, x_{1}\right)^{\lambda_{2}, \ldots, \lambda_{s}} x_{2}\right)^{\lambda_{1} \mathbf{F i}}\left(x_{1}, x_{2}\right)^{\lambda_{3}, \ldots, \lambda_{s}} \quad(s \geq 1) .
\end{gathered}
$$

In particular,

$$
\begin{gathered}
\mathbf{F i}\left(x_{1}, x_{2}\right)^{\lambda_{1}}=\left(x_{2}\right)^{\lambda_{1}}, \\
\mathbf{F i}\left(x_{1}, x_{2}\right)^{\lambda_{1}, \lambda_{2}}=\left(\left(x_{1}\right)^{\lambda_{2}} x_{2}\right)^{\lambda_{1}}, \\
{ }^{\mathbf{F i}}\left(x_{1}, x_{2}\right)^{\lambda_{1}, \lambda_{2}, \lambda_{3}}=\left(\left(\left(x_{2}\right)^{\lambda_{3}} x_{1}\right)^{\lambda_{2}} x_{2}\right)^{\lambda_{1}}\left(x_{2}\right)^{\lambda_{3}}, \\
{ }^{\mathbf{F i}}\left(x_{1}, x_{2}\right)^{\lambda_{1}, \lambda_{2}, \lambda_{3}, \lambda_{4}}=\left(\left(\left(\left(x_{1}\right)^{\lambda_{4}} x_{2}\right)^{\lambda_{3}} x_{1}\right)^{\lambda_{2}}\left(x_{1}\right)^{\lambda_{4}} x_{2}\right)^{\lambda_{1}}\left(\left(x_{1}\right)^{\lambda_{4}} x_{2}\right)^{\lambda_{3}} .
\end{gathered}
$$

The empty sequence is denoted by $\varnothing$. Let $\mu$ be a variable whose values are finite sequences of natural parameters. The variable $\mu \mid$ is connected with a variable $\mu$ as follows: If $\mu=\lambda_{1}, \lambda_{2}, \ldots, \lambda_{s}$, where $s \geq 1$, then $\mu \mid=\lambda_{2}, \ldots, \lambda_{s}$. If $\mu=\varnothing$, then $\mu \mid=\varnothing$.

A transformation is defined by the application

$$
\left\{\begin{array}{l}
x_{1} \rightarrow \mathbf{F i}\left(x_{1} P, x_{2} Q\right)^{\mu} x_{1}, \\
x_{2} \rightarrow \mathbf{F i}\left(x_{2} Q, x_{1} P\right)^{\mu \mid} x_{2},
\end{array}\right.
$$

where $P, Q$ are parametric words on the alphabet $x_{3}, \ldots, x_{n}$ and $\mu$ is a variable whose values are finite sequences of natural parameters, is a parametric transformation (by the function $\mathbf{F i}$ ).

We next define by a joint induction the functions

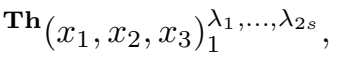

$$
\begin{aligned}
& \mathbf{T h}\left(x_{1}, x_{2}, x_{3}\right)_{2}^{\lambda_{1}, \ldots, \lambda_{2 s}} \text {, } \\
& \mathbf{T h}\left(x_{1}, x_{2}, x_{3}\right)_{3}^{\lambda_{1}, \ldots, \lambda_{2 s}},
\end{aligned}
$$

for $s \geq 0$, where $x_{1}, x_{2}, x_{3}$ are three word variables, and $\lambda_{1}, \ldots, \lambda_{2 s}$ are natural parameters. Specifically, we set

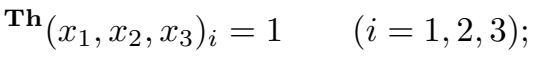

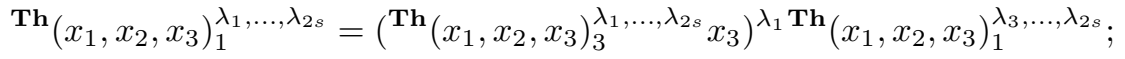

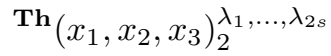

$$
\begin{aligned}
& =\left({ }^{\mathbf{T h}}\left(x_{1}, x_{2}, x_{3}\right)_{2}^{\lambda_{3}, \ldots, \lambda_{2 s}} x_{2}{ }^{\mathbf{T h}}\left(x_{1}, x_{2}, x_{3}\right)_{3}^{\lambda_{3}, \ldots, \lambda_{2 s}} x_{3}{ }^{\mathbf{T h}}\left(x_{1}, x_{2}, x_{3}\right)_{1}^{\lambda_{3}, \ldots, \lambda_{2 s}} x_{1}\right. \\
& \text {. Th } \left.\left(x_{1}, x_{2}, x_{3}\right)_{1}^{\lambda_{1}, \ldots, \lambda_{2 s}} x_{1}\right)^{\lambda_{2} \mathbf{T h}}\left(x_{1}, x_{2}, x_{3}\right)_{2}^{\lambda_{3}, \ldots, \lambda_{2 s}} \text {; }
\end{aligned}
$$

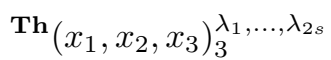

$$
\begin{aligned}
& ={ }^{\mathbf{T h}}\left(x_{1}, x_{2}, x_{3}\right)_{1}^{\lambda_{3}, \ldots, \lambda_{2 s}} x_{1}{ }^{\mathbf{T h}}\left(x_{1}, x_{2}, x_{3}\right)_{2}^{\lambda_{3}, \ldots, \lambda_{2 s}} x_{2}{ }^{\mathbf{T h}}\left(x_{1}, x_{2}, x_{3}\right)_{3}^{\lambda_{3}, \ldots, \lambda_{2 s}} .
\end{aligned}
$$


Define inductively the auxiliary function

$$
\text { Oc }\left(x_{1}, x_{2}, x_{3}\right)^{\lambda_{1}, \ldots, \lambda_{2 s}}
$$

for $s \geq 0$, where $x_{1}, x_{2}, x_{3}$ are three word variables, and $\lambda_{1}, \ldots, \lambda_{2 s}$ are natural parameters, by setting

$$
\begin{gathered}
\text { Oc }_{\left(x_{1}, x_{2}, x_{3}\right)=1} \\
\text { Oc }_{\left(x_{1}, x_{2}, x_{3}\right)^{\lambda_{1}, \ldots, \lambda_{2 s}}} \\
={ }^{\mathbf{O c}}\left(x_{1}, x_{2}, x_{3}\right)^{\lambda_{3}, \ldots, \lambda_{2 s}\left({ }^{\mathbf{T h}}\right.}\left(x_{1}, x_{2}, x_{3}\right)_{3}^{\lambda_{3}, \ldots, \lambda_{2 s}} x_{3} \\
\left..{ }^{\mathbf{T h}}\left(x_{1}, x_{2}, x_{3}\right)_{1}^{\lambda_{3}, \ldots, \lambda_{2 s}} x_{1}^{\mathbf{T h}}\left(x_{1}, x_{2}, x_{3}\right)_{2}^{\lambda_{3}, \ldots, \lambda_{2 s}} x_{2}\right)^{\lambda_{1}} .
\end{gathered}
$$

A transformation defined by the application

$$
\left\{\begin{array}{l}
x_{i} \rightarrow^{\mathbf{T h}}\left(x_{1}, x_{2}, x_{3}\right)_{i}^{\xi} x_{i}, \quad i=1,2,3, \\
x_{4} \rightarrow x_{4}^{\text {Oc }}\left(x_{1}, x_{2}, x_{3}\right)^{\xi},
\end{array}\right.
$$

where $\xi$ is a variable whose values are even sequences of natural parameters, is called a parametric transformation (by the function ${ }^{\mathbf{T h}}$ ).

Define by a joint induction the functions

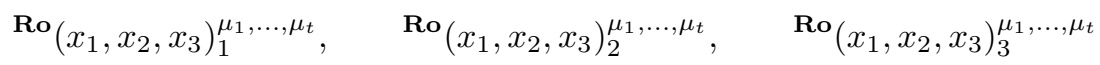

for $t \geq 0$, where $x_{1}, x_{2}, x_{3}$ are three word variables, and $\mu_{1}, \ldots, \mu_{t}$ are variables whose values are finite sequences of natural parameters, as follows:

$$
\begin{aligned}
& \text { Ro }\left(x_{1}, x_{2}, x_{3}\right)_{i}=1 \quad(i=1,2,3) ; \\
& \text { Ro }\left(x_{1}, x_{2}, x_{3}\right)_{1}^{\mu_{1}, \ldots, \mu_{t}} \\
& ={ }^{\mathbf{F i}}\left({ }^{\mathbf{R o}}\left(x_{2}, x_{1}, x_{3}\right)_{3}^{\mu_{2}, \ldots, \mu_{t}} x_{3}{ }^{\mathbf{R o}}\left(x_{2}, x_{1}, x_{3}\right)_{1}^{\mu_{2}, \ldots, \mu_{t}} x_{2},\right. \\
& \text {. }{ }^{\mathbf{R o}}\left(x_{2}, x_{1}, x_{3}\right)_{2}^{\mu_{2}, \ldots, \mu_{t}} x_{1}{ }^{\mathbf{R o}}\left(x_{2}, x_{1}, x_{3}\right)_{3}^{\mu_{2}, \ldots, \mu_{t}} x_{3} \\
& \text {. Ro } \left.\left(x_{2}, x_{1}, x_{3}\right)_{1}^{\mu_{2}, \ldots, \mu_{t}} x_{2}\left({ }^{\mathbf{R o}}\left(x_{1}, x_{2}, x_{3}\right)_{3}^{\mu_{1}, \ldots, \mu_{t}} x_{3}\right)^{2}\right)^{\mu_{1}} \\
& \text {. }{ }^{\mathbf{R o}}\left(x_{2}, x_{1}, x_{3}\right)_{3}^{\mu_{2}, \ldots, \mu_{t}} x_{3}{ }^{\mathbf{R o}}\left(x_{2}, x_{1}, x_{3}\right)_{1}^{\mu_{2}, \ldots, \mu_{t}} x_{2} \\
& \text {. Ro }\left(x_{2}, x_{1}, x_{3}\right)_{2}^{\mu_{2}, \ldots, \mu_{t}} \text {; } \\
& \text { Ro }\left(x_{1}, x_{2}, x_{3}\right)_{2}^{\mu_{1}, \ldots, \mu_{t}} \\
& ={ }^{\mathbf{F i}}\left({ }^{\mathbf{R o}}\left(x_{2}, x_{1}, x_{3}\right)_{3}^{\mu_{2}, \ldots, \mu_{t}} x_{3}{ }^{\mathbf{R o}}\left(x_{2}, x_{1}, x_{3}\right)_{1}^{\mu_{2}, \ldots, \mu_{t}} x_{2}\right. \\
& \text { - }\left({ }^{\mathbf{R o}}\left(x_{1}, x_{2}, x_{3}\right)_{3}^{\mu_{1}, \ldots, \mu_{t}} x_{3}\right)^{2},{ }^{\mathbf{R o}}\left(x_{2}, x_{1}, x_{3}\right)_{3}^{\mu_{2}, \ldots, \mu_{t}} x_{3} \\
& \text {. } \left.{ }^{\mathbf{R o}}\left(x_{2}, x_{1}, x_{3}\right)_{1}^{\mu_{2}, \ldots, \mu_{t}} x_{2}{ }^{\mathbf{R o}}\left(x_{2}, x_{1}, x_{3}\right)_{2}^{\mu_{2}, \ldots, \mu_{t}} x_{1}\right)^{\mu_{1} \mid} \\
& .{ }^{\mathbf{R o}}\left(x_{2}, x_{1}, x_{3}\right)_{3}^{\mu_{2}, \ldots, \mu_{t}} x_{3}{ }^{\mathbf{R o}}\left(x_{2}, x_{1}, x_{3}\right)_{2}^{\mu_{2}, \ldots, \mu_{t}}
\end{aligned}
$$

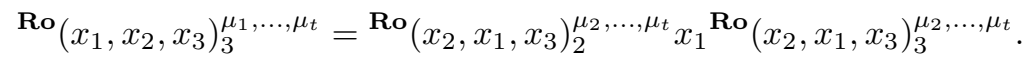

Define inductively the auxiliary function

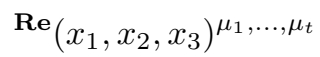

for $t \geq 0$, where $x_{1}, x_{2}, x_{3}$ are three word variables, and $\mu_{1}, \ldots, \mu_{t}$ are variables for sequences of natural parameters:

$$
\mathbf{R e}_{\left(x_{1}, x_{2}, x_{3}\right)=1}
$$




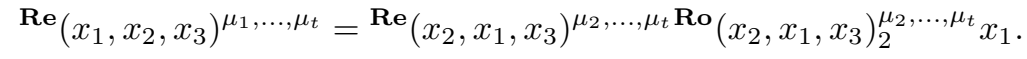

A transformation defined by the application

$$
\left\{\begin{array}{l}
x_{i} \rightarrow \mathbf{R o}\left(x_{1}, x_{2}, x_{3}\right)_{i}^{\nu} x_{i}, \quad i=1,2,3, \\
x_{4} \rightarrow x_{4}^{\mathbf{R e}}\left(x_{1}, x_{2}, x_{3}\right)^{\nu}
\end{array}\right.
$$

where $\nu$ is a variable whose values are finite sequences of second parameters, is a parametric transformation (by the function ${ }^{\mathbf{R o}}$ ).

A composition of parametric transformations is a parametric transformation. Any word in the right side of a parametric transformations is a parametric word.

A coefficient transformation is defined by the application

$$
\begin{cases}x_{i} \rightarrow X_{i} & (i=1, \ldots, n) \\ \lambda_{i} \rightarrow \Lambda_{i} & (i=1, \ldots, t) \\ \mu_{i} \rightarrow M_{i} & (i=1, \ldots, u) \\ \nu_{i} \rightarrow N_{i} & (i=1, \ldots, r)\end{cases}
$$

where every $X_{i}$ is a word in the alphabet (1), every $\Lambda_{i}$ is a natural number, every $M_{i}$ is a finite sequence of $\left(\lambda_{1}, \ldots, \lambda_{t}\right)$, and every $N_{i}$ is a finite sequence of $\left(\mu_{1}, \ldots, \mu_{u}\right)$.

A coefficient transformation

$$
\begin{cases}x_{i} \rightarrow X_{i} & (i=1, \ldots, n) \\ \lambda_{i} \rightarrow \Lambda_{i} & (i=1, \ldots, l) \\ \mu_{i} \rightarrow M_{i} & (i=1, \ldots, m) \\ \nu_{i} \rightarrow N_{i} & (i=1, \ldots, p)\end{cases}
$$

is called an extension of the coefficient transformation (6) if $l \geq t, m \geq u$, and $p \geq r$.

A parametric equation in a free monoid is given by an equality of parametric words

$$
\Phi\left(x_{1}, \ldots, x_{n}, \lambda_{1}, \ldots, \lambda_{t}\right)=\Psi\left(x_{1}, \ldots, x_{n}, \lambda_{1}, \ldots, \lambda_{t}\right) .
$$

If $\Phi$ and $\Psi$ are empty words, the equation (7) is called the trivial equation, denoted by 1 .

A parametric transformation (a coefficient transformation) is called a parametric solution (a solution) of the equation (7) if the result of the application of this transformation to $(7)$ is the trivial equation.

We will say that the parametric transformation $T$ contains the coefficient transformation $C$ by means of the auxiliary transformation $I$, if $T I=C$.

A finite list of parametric solutions of the equation $E$ will be called a general solution of $E$, if every solution of $E$ is contained in some parametric solution of this list. The general solution of $E$ will be denoted $\langle E\rangle$.

The length of a word $A$ in the alphabet (1) is denoted by $|A|$. The empty word is denoted by 1 . The length of a finite sequence $B$ is denoted by $|B|$.

A condition on natural parameters has the form

$$
L_{1}\left(\lambda_{1}, \ldots, \lambda_{q}\right)<, \leq,=L_{2}\left(\lambda_{1}, \ldots, \lambda_{q}\right)
$$

where $L_{1}, L_{2}$ are integer polynomials. A coefficient transformation (6) is called a solution of the equation $E$ with condition (8) on natural parameters, if (6) is a 
solution of $E$ that satisfies

$$
L_{1}\left(\Lambda_{1}, \ldots, \Lambda_{q}\right)<, \leq,=L_{2}\left(\Lambda_{1}, \ldots, \Lambda_{q}\right) .
$$

A condition on the length of a solution has the form

$$
0, \partial\left(P\left(x_{1}, \ldots, x_{n}, \lambda_{1}, \ldots, \lambda_{q}\right)\right)<, \leq,=\partial\left(Q\left(x_{1}, \ldots, x_{n}, \lambda_{1}, \ldots, \lambda_{q}\right)\right),
$$

where $P, Q$ are two primitive parametric words. A coefficient transformation (6) is called a solution of the equation $E$ with a condition (9) on the lengths of solutions, if (6) is a solution of $E$ that satisfies

$$
0,\left|P\left(X_{1}, \ldots, X_{n}, \Lambda_{1}, \ldots, \Lambda_{q}\right)\right|<, \leq,=\left|Q\left(X_{1}, \ldots, X_{n}, \Lambda_{1}, \ldots, \Lambda_{q}\right)\right| .
$$

A condition on the length of a sequence of variables has the form

$$
N_{i}<, \leq,=\partial\left(\mu_{i}\right)
$$

where $N_{i}$ is a natural number and $\mu_{i}$ is a second parameter. A coefficient transformation (6) is called a solution of the equation $E$ with a condition (10) on the length of sequences, if (6) is a solution of $E$ that satisfies

$$
N_{i}<, \leq,=\left|M_{i}\right| .
$$

A parametric transformation $T$ is called a parametric solution of the equation $E$ with a condition $R$, if $T$ is a parametric solution of $E$ and the result of the application of $T$ to $R$ is a true proposition for any values of the variables.

Let $E$ be an equation with conditions and let $R_{1}, \ldots, R_{m}$ be the list of new conditions. By $\left(E, R_{i}\right)$ we denote the equation $E$ with additional condition $R_{i}$. The equation $E$ s said to be divided into a collection of equations $\left(E, R_{1}\right), \ldots,\left(E, R_{m}\right)$, if every solution $S$ of $E$ is a solution of some $\left(E, R_{i}\right)$. An equation $\left(E, R_{1}\right)$ contains an equation $\left(E, R_{2}\right)$ (and we write $\left.\left(E, R_{1}\right) \supseteq\left(E, R_{2}\right)\right)$, if every solution of $\left(E, R_{2}\right)$ is a solution of $\left(E, R_{1}\right)$.

We say that the equation $E_{1}$ is reduced by the parametric transformation $T$ to the equation $E_{2}$, if $E_{1} T=E_{2}^{\prime}$, where $E_{2}^{\prime} \supseteq E_{2}$, and for every solution $S_{1}$ of $E_{1}$ there exists a solution $S_{2}$ of $E_{2}$ such that $S_{1}=T S_{2}^{*}$ for some extension $S_{2}^{*}$ of $S_{2}$. We say that $S_{2}$ is the image of $S_{1}$ via the transformation $T$. We need the extension $S_{2}^{*}$, because $T$ could have some variables that are not in $E_{2}$.

Lemma 1. Let $E_{1}$ be reduced by $T$ to $E_{2}$. Let $S_{1}$ be a solution of $E_{1}$ and $S_{2}$ its image via $T$. If the parametric solution $Q_{2}$ of $E_{2}$ contains a solution $S_{2}$ of $E_{2}$, then the parametric solution $T Q_{2}$ of $E_{1}$ contains the solution $S_{1}$ of $E_{1}$.

Theorem 1. Let the equation $E_{1}$ be reduced by the parametric transformation $T$ to the equation $E_{2}$. If the general solution $\left\langle E_{2}\right\rangle$ of $E_{2}$ is $Q_{1}, \ldots, Q_{r}$, then the general solution $\left\langle E_{1}\right\rangle$ of $E_{1}$ is $T Q_{1}, \ldots, T Q_{r}$.

Theorem 2. Let the equation $E$ be divided into a collection of equations with conditions $\left(E, R_{1}\right), \ldots,\left(E, R_{m}\right)$. If the general solution $\left\langle\left(E, R_{i}\right)\right\rangle$ of $\left(E, R_{i}\right)$ is $Q_{i, 1}, \ldots, Q_{i, r_{i}}(i=1, \ldots, m)$, then the general solution $\langle E\rangle$ of $E$ is $Q_{1,1}, \ldots, Q_{1, r_{1}}$, $\ldots, Q_{m 1}, \ldots, Q_{m, r_{m}}$.

Let

$$
K_{\alpha}\left(\lambda_{1}, \ldots, \lambda_{q}\right)<, \leq,=M_{\alpha}\left(\lambda_{1}, \ldots, \lambda_{q}\right) \quad(\alpha=1, \ldots, t),
$$


where $K_{\alpha}, M_{\alpha} \in \mathbf{L}$, be a system of linear Diophantine equations and inequations. A transformation

$$
\lambda_{i} \rightarrow L_{i}, \quad L_{i} \in \mathbf{L} \quad(i=1, \ldots, q),
$$

is called a parametric solution of the system (11), if

$$
K_{\alpha}\left(L_{1}, \ldots, L_{q}\right)<, \leq,=M_{\alpha}\left(L_{1}, \ldots, L_{q}\right) \quad(\alpha=1, \ldots, t)
$$

for any values of the variables.

Theorem 3. The family of solutions of the system (11) is described by a finite list of parametric solution (see [7]).

\section{Preliminaries}

The following seven propositions belong to folklore (see [4], [8], [9]). Observe that a boldface $\boldsymbol{n}$ means "the equation in Proposition $\boldsymbol{n}$ ".

Proposition 1. The general solution of the equation

1

$$
x_{1} x_{2}=x_{2} x_{1}
$$

is described by the transformation

$$
\left\{\begin{array}{l}
x_{1} \rightarrow x_{1}^{\alpha} \\
x_{2} \rightarrow x_{1}^{\beta}
\end{array}\right.
$$

where $\alpha, \beta$ are natural parameters.

Proposition 2. The general solution of the equation

$$
2
$$$$
x_{1} x_{2} x_{3}=x_{3} x_{1} x_{2}
$$

is described by the transformations

$$
\left\{\begin{array} { l } 
{ x _ { 1 } \rightarrow 1 , } \\
{ x _ { 2 } \rightarrow 1 , } \\
{ x _ { 3 } \rightarrow x _ { 3 } , }
\end{array} \quad \left\{\begin{array}{l}
x_{1} \rightarrow\left(x_{1} x_{2}\right)^{\alpha} x_{1}, \\
x_{2} \rightarrow\left(x_{2} x_{1}\right)^{\beta} x_{2}, \\
x_{3} \rightarrow\left(x_{1} x_{2}\right)^{\gamma},
\end{array}\right.\right.
$$

where $\alpha, \beta, \gamma$ are natural parameters.

Proposition 3. The general solution of the equation

3

$$
x_{1} x_{2} x_{3}=x_{2}^{\alpha} x_{1}
$$

where $\alpha$ is a natural parameter, is described by the transformations

$$
\left\{\begin{array}{l}
x_{1} \rightarrow x_{1}^{\beta} \\
x_{2} \rightarrow x_{1}^{\gamma} \\
x_{3} \rightarrow x_{1}^{\delta}
\end{array}\right.
$$

where $\beta, \gamma, \delta$ are natural parameters.

Proposition 4. The general solution of the equation

4

is described by the transformations

$$
\left\{\begin{array} { l } 
{ x _ { 1 } \rightarrow x _ { 1 } , } \\
{ x _ { 2 } \rightarrow 1 , } \\
{ x _ { 3 } \rightarrow 1 , }
\end{array} \quad \left\{\begin{array}{l}
x_{1} \rightarrow\left(x_{1} x_{2}\right)^{\alpha} x_{1}, \\
x_{2} \rightarrow x_{1} x_{2}, \\
x_{3} \rightarrow x_{2} x_{1},
\end{array}\right.\right.
$$

where $\alpha$ is a natural parameter.

$$
x_{1} x_{3}=x_{2} x_{1}
$$


Proposition 5. The general solution of the equation

5

$$
x_{1} x_{3}=x_{2}^{\alpha} x_{1},
$$

where $\alpha$ is a natural parameter, is described by the transformations

$$
\left\{\begin{array} { l } 
{ x _ { 1 } \rightarrow x _ { 1 } , } \\
{ x _ { 2 } \rightarrow x _ { 2 } , } \\
{ x _ { 3 } \rightarrow 1 , } \\
{ \alpha \rightarrow 0 , }
\end{array} \quad \left\{\begin{array} { l } 
{ x _ { 1 } \rightarrow x _ { 1 } , } \\
{ x _ { 2 } \rightarrow 1 , } \\
{ x _ { 3 } \rightarrow 1 , } \\
{ \alpha \rightarrow \alpha , }
\end{array} \quad \left\{\begin{array}{l}
x_{1} \rightarrow\left(x_{1} x_{2}\right)^{\beta} x_{1}, \\
x_{2} \rightarrow x_{1} x_{2}, \\
x_{3} \rightarrow\left(x_{2} x_{1}\right)^{\alpha} \\
\alpha \rightarrow \alpha
\end{array}\right.\right.\right.
$$

where $\beta$ is a natural parameter.

Proposition 6. The general solution of the equation

6

$$
x_{1} x_{2} x_{3}=x_{3} x_{4}
$$

is described by the transformations

$$
\left\{\begin{array} { l } 
{ x _ { 1 } \rightarrow 1 , } \\
{ x _ { 2 } \rightarrow 1 , } \\
{ x _ { 3 } \rightarrow x _ { 3 } , } \\
{ x _ { 4 } \rightarrow 1 , }
\end{array} \quad \left\{\begin{array} { l } 
{ x _ { 1 } \rightarrow x _ { 1 } , } \\
{ x _ { 2 } \rightarrow x _ { 3 } x _ { 2 } , } \\
{ x _ { 3 } \rightarrow ( x _ { 1 } x _ { 3 } x _ { 2 } ) ^ { \alpha } x _ { 1 } x _ { 3 } , } \\
{ x _ { 4 } \rightarrow x _ { 2 } x _ { 1 } x _ { 3 } , }
\end{array} \quad \left\{\begin{array}{l}
x_{1} \rightarrow x_{3} x_{1}, \\
x_{2} \rightarrow x_{2}, \\
x_{3} \rightarrow\left(x_{3} x_{1} x_{2}\right)^{\alpha} x_{3}, \\
x_{4} \rightarrow x_{1} x_{2} x_{3},
\end{array}\right.\right.\right.
$$

where $\alpha$ is a natural parameter.

Proposition 7. The general solution of the equation

7

$$
x_{1} x_{2} x_{3}=x_{2} x_{3} x_{4}
$$

is described by the transformations

$$
\left\{\begin{array} { l } 
{ x _ { 1 } \rightarrow 1 , } \\
{ x _ { 2 } \rightarrow x _ { 2 } , } \\
{ x _ { 3 } \rightarrow x _ { 3 } , } \\
{ x _ { 4 } \rightarrow 1 , }
\end{array} \quad \left\{\begin{array} { l } 
{ x _ { 1 } \rightarrow x _ { 2 } x _ { 3 } x _ { 1 } , } \\
{ x _ { 2 } \rightarrow ( x _ { 2 } x _ { 3 } x _ { 1 } ) ^ { \alpha } x _ { 2 } , } \\
{ x _ { 3 } \rightarrow ( x _ { 3 } x _ { 1 } x _ { 2 } ) ^ { \beta } x _ { 3 } , } \\
{ x _ { 4 } \rightarrow x _ { 1 } x _ { 2 } x _ { 3 } , }
\end{array} \quad \left\{\begin{array}{l}
x_{1} \rightarrow x_{3} x_{2} x_{1}, \\
x_{2} \rightarrow\left(x_{3} x_{2} x_{1}\right)^{\alpha} x_{3} x_{2}, \\
x_{3} \rightarrow\left(x_{1} x_{3} x_{2}\right)^{\beta} x_{1} x_{3}, \\
x_{4} \rightarrow x_{2} x_{1} x_{3},
\end{array}\right.\right.\right.
$$

where $\alpha, \beta$ are natural parameters.

Proposition 8. The parametric equation

8

$$
x_{1} R\left(x_{2}, x_{3}\right) x_{4}=\left(p\left(x_{2}, x_{3}\right)\right)^{t+1} x_{1} Q\left(x_{1}, x_{2}, x_{3}\right) x_{5}
$$

with $\partial\left(P\left(x_{2}, x_{3}\right)\right)>0$, where $P, Q, R$ are parametric words and $t$ is a natural number, is reduced by the parametric transformation $T$ :

$$
x_{1} \rightarrow\left(P\left(x_{2}, x_{3}, \lambda_{1}, \ldots, \lambda_{r}\right)\right)^{\alpha} x_{1},
$$

where $\alpha$ is a natural parameter, to the parametric equation $E$ :

$$
\begin{aligned}
& x_{1} R\left(x_{2}, x_{3}\right) x_{4} \\
& \left.\quad=\left(P\left(x_{2}, x_{3}, \lambda_{1}, \ldots, \lambda_{r}\right)\right)^{t+1} x_{1} Q\left(P\left(x_{2}, x_{3}, \lambda_{1}, \ldots, \lambda_{r}\right)\right)^{\alpha} x_{1}, x_{2}, x_{3}, \lambda_{1}, \ldots, \lambda_{r}\right) x_{5} \\
& \text { with } \partial\left(x_{1}\right)<\partial\left(P\left(x_{2}, x_{3}, \lambda_{1}, \ldots, \lambda_{r}\right)\right) .
\end{aligned}
$$


Proof. It is easy to verify that the substitution of the transformation $T$ into $\mathbf{8}$ transforms 8 to some equation $E^{\prime}$ which contains $E$. On the other hand, let the transformation $S_{1}$ :

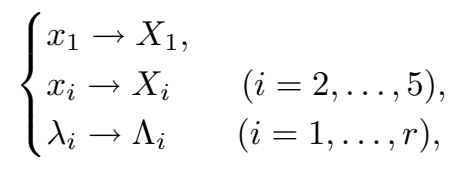

where the $X_{i}$ are words in the alphabet (1) and the $\Lambda_{i}$ are natural numbers, be an arbitrary solution of the equation $\mathbf{8}$. Since $\left|P\left(X_{2}, X_{3}, \Lambda_{1}, \ldots, \Lambda_{r}\right)\right|>0$, we have $X_{1}=\left(P\left(X_{2}, X_{3}, \Lambda_{1}, \ldots, \Lambda_{r}\right)\right)^{A} Y_{1}$ for some word $Y_{1}$ in the alphabet (1) and some natural number $A$ such that

$$
A\left|P\left(X_{2}, X_{3}, \Lambda_{1}, \ldots, \Lambda_{r}\right)\right| \leq\left|X_{1}\right|<(A+1)\left|P\left(X_{2}, X_{3}, \Lambda_{1}, \ldots, \Lambda_{r}\right)\right| .
$$

After the substitution of the solution $S_{1}$ in the equation we easily obtain that the coefficient transformation $S_{2}$ :

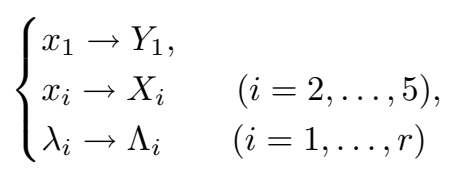

is a solution of the equation $E$. The extension $S_{2}^{*}$ :

$$
\left\{\begin{array}{l}
x_{1} \rightarrow Y_{1} \\
x_{i} \rightarrow X_{i} \\
\lambda_{i} \rightarrow \Lambda_{i} \\
\alpha \rightarrow A
\end{array} \quad(i=2, \ldots, 5),\right.
$$

satisfies $S_{1}=T S_{2}^{*}$. Therefore the solution $S_{2}$ is the image of $S_{1}$ via $T$. Thus the parametric equation 8 is reduced by the parametric transformation $T$ to the parametric equation $E$.

\section{The funCtion ${ }^{\mathbf{F i}}\left(x_{1}, x_{2}\right)^{\lambda_{1}, \ldots, \lambda_{s}}$}

Theorem ${ }^{\mathbf{F i}} \mathbf{1}$. The following identities hold:

$$
\begin{gathered}
\mathbf{F i}\left(x_{1}, x_{2}\right)^{\lambda_{1}, \ldots, \lambda_{2 k}}={ }^{\mathbf{F i}}\left(x_{1}, x_{1}^{\lambda_{2 k}} x_{2}\right)^{\lambda_{1}, \ldots, \lambda_{2 k-1}} \quad(k \geq 1), \\
{ }^{\mathbf{F i}}\left(x_{1}, x_{2}\right)^{\lambda_{1}, \ldots, \lambda_{2 k+1}}={ }^{\mathbf{F i}}\left(x_{2}^{\lambda_{2 k+1}} x_{1}, x_{2}\right)^{\lambda_{1}, \ldots, \lambda_{2 k}} x_{2}^{\lambda_{2 k+1}} \quad(k \geq 0) .
\end{gathered}
$$

Proof (Joint induction on $k$ ). If $k=0$ or 1 , the proof is obvious.

Suppose that $k>1$. By definition, ${ }^{\mathbf{F i}}\left(x_{1}, x_{2}\right)^{\lambda_{1}, \ldots, \lambda_{2 k}}$ equals

$$
\left({ }^{\mathbf{F i}}\left(x_{2}, x_{1}\right)^{\lambda_{2}, \ldots, \lambda_{2 k}} x_{2}\right)^{\lambda_{1} \mathbf{F i}}\left(x_{1}, x_{2}\right)^{\lambda_{3}, \ldots, \lambda_{2 k}} .
$$

According to the induction proposition (second identity), this last expression equals

$$
\left({ }^{\mathbf{F i}}\left(x_{1}^{\lambda_{2 k}} x_{2}, x_{1}\right)^{\lambda_{2}, \ldots, \lambda_{2 k-1}} x_{1}^{\lambda_{2 k}} x_{2}\right)^{\lambda_{1} \mathbf{F i}}\left(x_{1}, x_{2}\right)^{\lambda_{3}, \ldots, \lambda_{2 k}} .
$$

According to the induction proposition (first identity), this in turn is equal to

$$
\left({ }^{\mathbf{F i}}\left(x_{1}^{\lambda_{2 k}} x_{2}, x_{1}\right)^{\lambda_{2}, \ldots, \lambda_{2 k-1}} x_{1}^{\lambda_{2 k}} x_{2}\right)^{\lambda_{1} \mathbf{F i}}\left(x_{1}, x_{1}^{\lambda_{2 k}} x_{2}\right)^{\lambda_{3}, \ldots, \lambda_{2 k-1}},
$$

and this is equal (by definition) to

$$
{ }^{\mathbf{F i}}\left(x_{1}, x_{1}^{\lambda_{2 k}} x_{2}\right)^{\lambda_{1}, \ldots, \lambda_{2 k-1}} .
$$


By definition, ${ }^{\mathbf{F i}}\left(x_{1}, x_{2}\right)^{\lambda_{1}, \ldots, \lambda_{2 k+1}}$ equals

$$
\left({ }^{\mathbf{F i}}\left(x_{2}, x_{1}\right)^{\lambda_{2}, \ldots, \lambda_{2 k+1}} x_{2}\right)^{\lambda_{1} \mathbf{F i}}\left(x_{1}, x_{2}\right)^{\lambda_{3}, \ldots, \lambda_{2 k+1}} .
$$

According to the induction proposition (first identity), this last expression equals

$$
\left({ }^{\mathbf{F i}}\left(x_{2}, x_{2}^{\lambda_{2 k+1}} x_{1}\right)^{\lambda_{2}, \ldots, \lambda_{2 k}} x_{2}\right)^{\lambda_{1} \mathbf{F i}}\left(x_{1}, x_{2}\right)^{\lambda_{3}, \ldots, \lambda_{2 k+1}},
$$

and by the induction proposition (second identity), this is in turn equal to

$$
\left.{ }^{\mathbf{F i}}\left(x_{2}, x_{2}^{\lambda_{2 k+1}} x_{1}\right)^{\lambda_{2}, \ldots, \lambda_{2 k}} x_{2}\right)^{\lambda_{1} \mathbf{F i}}\left(x_{2}^{\lambda_{2 k+1}} x_{1}, x_{2}\right)^{\lambda_{3}, \ldots, \lambda_{2 k}} x_{2}^{\lambda_{2 k+1}} .
$$

But this is equal (by definition) to

$$
\mathbf{F i}\left(x_{2}^{\lambda_{2 k+1}} x_{1}, x_{2}\right)^{\lambda_{1}, \ldots, \lambda_{2 k}} x_{2}^{\lambda_{2 k+1}} .
$$

Consider an equation of the form $x_{1} P x_{2} U=x_{2} Q x_{1} V$, where $P, Q$ are parametric words in the alphabet $x_{3}, \ldots, x_{n}$ and $U, V$ are parametric words. Consider the sequence of parametric transformations

$$
\begin{aligned}
& \text { 1. } \quad x_{1} \rightarrow\left(x_{2} Q\right)^{\lambda_{1}} x_{1} \text {, } \\
& \text { 2. } \quad x_{2} \rightarrow\left(x_{1} P\right)^{\lambda_{2}} x_{2} \text {, } \\
& \text { 3. } \quad x_{1} \rightarrow\left(x_{2} Q\right)^{\lambda_{3}} x_{1} \text {, } \\
& \text { 4. } \quad x_{2} \rightarrow\left(x_{1} P\right)^{\lambda_{4}} x_{2} \text {, } \\
& \text {.......................... } \\
& 2 k-1 . \quad x_{1} \rightarrow\left(x_{2} Q\right)^{\lambda_{2 k-1}} x_{1}, \\
& 2 k . \quad x_{2} \rightarrow\left(x_{1} P\right)^{\lambda_{2 k}} x_{2}, \\
& 2 k+1 . \quad x_{1} \rightarrow\left(x_{2} Q\right)^{\lambda_{2 k+1}} x_{1}, \\
& \text {........................ }
\end{aligned}
$$

where $\lambda_{1}, \lambda_{2}, \ldots$ are natural parameters.

Theorem ${ }^{\mathbf{F i}} \mathbf{2}$. For every natural $s$, the sequence (12) of the first $s$ parametric transformation can be collected by the following common transformation:

$$
\left\{\begin{array}{l}
x_{1} \rightarrow{ }^{\mathbf{F i}}\left(x_{1} P, x_{2} Q\right)^{\lambda_{1}, \ldots, \lambda_{s}} x_{1}, \\
x_{2} \rightarrow \mathbf{F i}\left(x_{2} Q, x_{1} P\right)^{\lambda_{2}, \ldots, \lambda_{s}} x_{2} .
\end{array}\right.
$$

Proof. If $s=0$ the proposition obviously holds. Consider two cases.

Case 1. Suppose that the sequence of the first $2 k-1$ transformations can be collected by the common transformation

$$
\left\{\begin{array}{l}
x_{1} \rightarrow \mathbf{F i}\left(x_{1} P, x_{2} Q\right)^{\lambda_{1}, \ldots, \lambda_{2 k-1}} x_{1}, \\
x_{2} \rightarrow \mathbf{F i}\left(x_{2} Q, x_{1} P\right)^{\lambda_{2}, \ldots, \lambda_{2 k-1}} x_{2} .
\end{array}\right.
$$

Let the $2 k$ th transformation be of the form

$$
x_{2} \rightarrow x_{2} P^{\lambda_{2 k}} x_{2}
$$

Then the sequence of the first $2 k$ transformations can be collected by the common transformation

$$
\left\{\begin{array}{l}
x_{1} \rightarrow{ }^{\mathbf{F i}}\left(x_{1} P,\left(x_{1} P\right)^{\lambda_{2 k}} x_{2} Q\right)^{\lambda_{1}, \ldots, \lambda_{2 k-1}} x_{1}, \\
x_{2} \rightarrow{ }^{\mathbf{F i}}\left(\left(x_{1} P\right)^{\lambda_{2 k}} x_{2} Q, x_{1} P\right)^{\lambda_{2}, \ldots, \lambda_{2 k-1}}\left(x_{1} P\right)^{\lambda_{2 k}} x_{2} .
\end{array}\right.
$$

According to Theorem ${ }^{\mathbf{F i}} 1$ this transformation coincides with the transformation

$$
\left\{\begin{array}{l}
x_{1} \rightarrow \mathbf{F i}\left(x_{1} P, x_{2} Q\right)^{\lambda_{1}, \ldots, \lambda_{2 k}} x_{1}, \\
x_{2} \rightarrow \mathbf{F i}\left(x_{2} Q, x_{1} P\right)^{\lambda_{2}, \ldots, \lambda_{2 k}} x_{2} .
\end{array}\right.
$$


Case 2. Suppose that the sequence of the first $2 k$ transformations can be collected in the common transformation

$$
\left\{\begin{array}{l}
x_{1} \rightarrow{ }^{\mathbf{F i}}\left(x_{1} P, x_{2} Q\right)^{\lambda_{1}, \ldots, \lambda_{2 k}} x_{1}, \\
x_{2} \rightarrow \mathbf{F i}\left(x_{2} Q, x_{1} P\right)^{\lambda_{2}, \ldots, \lambda_{2 k}} x_{2} .
\end{array}\right.
$$

Let the $(2 k+1)$ st transformation be of the form

$$
x_{1} \rightarrow\left(x_{2} Q\right)^{\lambda_{2 k+1}} x_{1} .
$$

Then the sequence of the first $2 k+1$ transformations can be collected by the common transformation

$$
\left\{\begin{array}{l}
x_{1} \rightarrow \mathbf{F i}\left(\left(x_{2} Q\right)^{\lambda_{2 k+1}} x_{1} P, x_{2} Q\right)^{\lambda_{1}, \ldots, \lambda_{2 k}}\left(x_{2} Q\right)^{\lambda_{2 k+1}} x_{1}, \\
x_{2} \rightarrow \mathbf{F i}\left(x_{2} Q,\left(x_{2} Q\right)^{\lambda_{2 k+1}} x_{1} P\right)^{\lambda_{2}, \ldots, \lambda_{2 k}} x_{2} .
\end{array}\right.
$$

According to Theorem ${ }^{\mathbf{F i}} 1$ this transformation coincides with the transformation

$$
\left\{\begin{array}{l}
x_{1} \rightarrow^{\mathbf{F i}}\left(x_{1} P, x_{2} Q\right)^{\lambda_{1}, \ldots, \lambda_{2 k+1}} x_{1}, \\
x_{2} \rightarrow^{\mathbf{F i}}\left(x_{2} Q, x_{1} P\right)^{\lambda_{2}, \ldots, \lambda_{2 k+1}} x_{2} .
\end{array}\right.
$$

Theorem ${ }^{\mathbf{F i} 3}$. The following identities hold:

$$
\begin{aligned}
{ }^{\mathbf{F i}}\left(x_{1}, x_{2}\right)^{0, \lambda_{2}, \ldots, \lambda_{s}} & ={ }^{\mathbf{F i}}\left(x_{1}, x_{2}\right)^{\lambda_{3}, \ldots, \lambda_{s}}, \\
\mathbf{F i}\left(x_{1}, x_{2}\right)^{\lambda_{1}, \ldots, \lambda_{r-1}, 0, \lambda_{r+1}, \ldots, \lambda_{s}} & ={ }^{\mathbf{F i}}\left(x_{1}, x_{2}\right)^{\lambda_{1}, \ldots, \lambda_{r-1}+\lambda_{r+1}, \ldots, \lambda_{s}}, \\
{ }^{\mathbf{F i}}\left(x_{1}, x_{2}\right)^{\lambda_{1}, \ldots, \lambda_{s-1}, 0} & ={ }^{\mathbf{F i}}\left(x_{1}, x_{2}\right)^{\lambda_{1}, \ldots, \lambda_{s-1}} .
\end{aligned}
$$

Proof. Follows from Theorem ${ }^{\mathbf{F i}} 2$.

Proposition 9. The parametric equation

$9 \quad x_{1} x_{3}^{\alpha} x_{2} U\left(x_{1}, x_{2}, x_{3}, \lambda_{1}, \ldots, \lambda_{r}\right) x_{4}=x_{2} x_{3}^{\beta} x_{1} V\left(x_{1}, x_{2}, x_{3}, \lambda_{1}, \ldots, \lambda_{r}\right) x_{5}$

with $\partial\left(x_{1} x_{3}^{\alpha}\right)>0, \partial\left(x_{2} x_{3}^{\beta}\right)>0, \alpha+\beta>0$, where $\alpha, \beta, \lambda_{1}, \ldots, \lambda_{r}$ are natural parameters and $U, V$ are parametric words, is reduced by the transformation $T$ :

$$
\left\{\begin{array}{l}
x_{1} \rightarrow \mathbf{F i}\left(x_{1} x_{3}^{\alpha}, x_{2} x_{3}^{\beta}\right)^{\mu} x_{1} \\
x_{2} \rightarrow \mathbf{F i}\left(x_{2} x_{3}^{\beta}, x_{1} x_{3}^{\alpha}\right)^{\mu \mid} x_{2}
\end{array}\right.
$$

where $\mu$ is a variable for sequences of natural parameters, to the equation $E$ :

$$
\begin{aligned}
& x_{1} x_{3}^{\alpha} x_{2} U\left({ }^{\mathbf{F i}}\left(x_{1} x_{3}^{\alpha}, x_{2} x_{3}^{\beta}\right)^{\mu} x_{1},{ }^{\mathbf{F i}}\left(x_{2} x_{3}^{\beta}, x_{1} x_{3}^{\alpha}\right)^{\mu \mid} x_{2}, x_{3}, \lambda_{1}, \ldots, \lambda_{r}\right) x_{4} \\
& \quad=x_{2} x_{3}^{\beta} x_{1} V\left({ }^{\mathbf{F i}}\left(x_{1} x_{3}^{\alpha}, x_{2} x_{3}^{\beta}\right)^{\mu} x_{1},{ }^{\mathbf{F i}}\left(x_{2} x_{3}^{\beta}, x_{1} x_{3}^{\alpha}\right)^{\mu \mid} x_{2}, x_{3}, \lambda_{1}, \ldots, \lambda_{r}\right) x_{5}
\end{aligned}
$$

with $\partial\left(x_{1}\right)<\partial\left(x_{2} x_{3}^{\beta}\right), \partial\left(x_{2}\right)<\partial\left(x_{1} x_{3}^{\alpha}\right)$.

Proof. It is easy to verify that $T$ reduces 9 to some equation $E^{\prime}$ which contains $E$.

On the other hand, let the transformation $S_{1}$ :

$$
\left\{\begin{array}{l}
x_{1} \rightarrow X_{i} \quad(i=1, \ldots, 5), \\
\lambda_{1} \rightarrow \Lambda_{i} \quad(i=1, \ldots, r), \\
\alpha \rightarrow A, \\
\beta \rightarrow B
\end{array}\right.
$$

where the $X_{i}$ are words in the alphabet (1) and $\Lambda_{i}, A, B$ are natural numbers, be an arbitrary solution of the equation $\mathbf{9}$. 
We prove by induction on $\left|X_{1} X_{2} X_{3}\right|$ that $S_{1}$ is contained in the parametric transformation $T$.

If $\left|X_{1}\right|<\left|X_{2} X_{3}^{B}\right|$ and $\left|X_{2}\right|<\left|X_{1} X_{3}^{A}\right|$, then the coefficient transformation $S_{2}$ :

$$
\left\{\begin{array}{l}
x_{i} \rightarrow X_{i} \quad(i=1, \ldots, 5), \\
\lambda_{i} \rightarrow \Lambda_{i} \quad(i=1, \ldots, r), \\
\alpha \rightarrow A \\
\beta \rightarrow B \\
\mu \rightarrow \varnothing
\end{array}\right.
$$

is a solution of the equation $E$, and $S_{1}=T S_{2}$.

Let $\left|X_{1}\right| \geq\left|X_{2} X_{3}^{B}\right|$. Since $\left|X_{2} X_{3}^{B}\right|>0$, we have $X_{1}=X_{2} X_{3}^{B} Y_{1}$ for some word $Y_{1}$ in the alphabet (1), where $\left|Y_{1}\right|<\left|X_{1}\right|$.

It is easy to see that equation $\mathbf{9}$ with the additional condition $\partial\left(x_{1}\right)>\partial\left(x_{2} x_{3}^{\beta}\right)$ is reduced by the transformation $t$ :

$$
x_{1} \rightarrow x_{2} x_{3}^{\beta} x_{1}
$$

to the equation $E^{\prime}$ :

$$
\begin{aligned}
& x_{1} x_{3}^{\alpha} x_{2} U\left(x_{2} x_{3}^{\beta} x_{1}, x_{2}, x_{3}, \lambda_{1}, \ldots, \lambda_{r}\right) x_{4} \\
& \quad=x_{2} x_{3}^{\beta} x_{1} V\left(x_{2} x_{3}^{\beta} x_{1}, x_{2}, x_{3}, \lambda_{1}, \ldots, \lambda_{r}\right) x_{5}
\end{aligned}
$$

with $\partial\left(x_{1} x_{3}^{\alpha}\right)>0, \partial\left(x_{2} x_{3}^{\beta}\right)>0, \alpha+\beta>0$.

The transformation $S^{\prime}$ :

$$
\left\{\begin{array}{l}
x_{1} \rightarrow Y_{1} \\
x_{i} \rightarrow X_{i} \\
\alpha \rightarrow A \\
\beta \rightarrow B
\end{array} \quad(i=2,3,4,5)\right.
$$

is a solution of the equation $E^{\prime}$.

Since $\left|Y_{1}\right|<\left|X_{1}\right|$, one can use the inductive proposition to see that $S^{\prime}$ is contained in the parametric transformation $T$. Hence $S_{1}$ is contained in the parameter solution $t T$. Using Theorem ${ }^{\mathbf{F i}_{2}}$, one can see that the transformation $t T$ has the form

$$
\left\{\begin{array}{l}
x_{1} \rightarrow^{\mathbf{F i}}\left(x_{1} x_{3}^{\alpha}, x_{2} x_{3}^{\beta}\right)^{1,0, \mu} x_{1}, \\
x_{2} \rightarrow^{\mathbf{F i}}\left(x_{2} x_{3}^{\beta}, x_{1} x_{3}^{\alpha}\right)^{\mu \mid} x_{2} .
\end{array}\right.
$$

Let $\left|X_{2}\right| \geq\left|X_{1} X_{3}^{A}\right|$. Since $\left|X_{1} X_{3}^{A}\right|>0$, we have $X_{2}=X_{1} X_{3}^{A} Y_{2}$ for some word $Y_{2}$ in the alphabet (1), where $\left|Y_{2}\right|<\left|X_{2}\right|$.

It is easy to see that equation $\mathbf{9}$ with the additional condition $\partial\left(x_{2}\right)>\partial\left(x_{1} x_{3}^{\alpha}\right)$ is reduced by the transformation $t: x_{2} \rightarrow x_{1} x_{3}^{\alpha} x_{2}$ to the equation $E^{\prime}$ :

$$
\begin{aligned}
& x_{1} x_{3}^{\alpha} x_{2} U\left(x_{1}, x_{1} x_{3}^{\alpha} x_{2}, x_{3}, \lambda_{1}, \ldots, \lambda_{r}\right) x_{4} \\
& \quad=x_{2} x_{3}^{\beta} x_{1} V\left(x_{1}, x_{1} x_{3}^{\alpha} x_{2}, x_{3}, \lambda_{1}, \ldots, \lambda_{r}\right) x_{5}
\end{aligned}
$$

with $\partial\left(x_{1} x_{3}^{\alpha}\right)>0, \partial\left(x_{2} x_{3}^{\beta}\right)>0, \alpha+\beta>0$. 
The transformation $S^{\prime}$ :

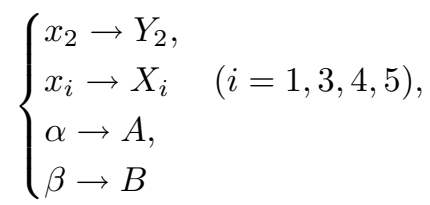

is a solution of $E^{\prime}$.

Since $\left|Y_{2}\right|<\left|X_{2}\right|$, one can use the inductive proposition to see that $S^{\prime}$ is contained in the parametric transformation $T$. Hence $S_{1}$ is contained in $t T$. Using Theorem $\mathbf{F i}_{2}$, one can see that the transformation $t T$ has form

$$
\left\{\begin{array}{l}
x_{1} \rightarrow \mathbf{F i}\left(x_{1} x_{3}^{\alpha}, x_{2} x_{3}^{\beta}\right)^{\mu} x_{1}, \\
x_{2} \rightarrow \mathbf{F i}\left(x_{2} x_{3}^{\beta}, x_{1} x_{3}^{\alpha}\right)^{1, \mu} x_{2} .
\end{array}\right.
$$

Proposition 10*. The general solution of the equation

$10^{*}$

$$
x_{1} x_{3}^{\alpha+1} x_{2}=x_{2} x_{1} x_{4} \quad \text { with } \partial\left(x_{1}\right)<\partial\left(x_{2}\right)<\partial\left(x_{1} x_{3}^{\alpha+1}\right),
$$

where $\alpha$ is a natural parameter, is described by the transformation

$$
\begin{gathered}
\left\{\begin{array}{l}
x_{2} \rightarrow x_{1}\left(x_{2} x_{3}\right)^{\beta} x_{2}, \\
x_{3} \rightarrow x_{2} x_{3},
\end{array}\right. \\
x_{1} \rightarrow\left(x_{3}\left(x_{2} x_{3}\right)^{\alpha-\beta}\right)^{\lambda} x_{1},
\end{gathered}
$$

followed by one of the two transformations

$$
\begin{aligned}
& \left\{\begin{array}{l}
x_{1} \rightarrow\left(x_{1} x_{3} x_{2}\right)^{\sigma} x_{1}, \\
x_{3} \rightarrow x_{1} x_{3} \\
x_{4} \rightarrow x_{3}\left(x_{2} x_{1} x_{3}\right)^{\alpha-\beta-\sigma}\left(x_{1} x_{3} x_{2}\right)^{\sigma} x_{1}\left(x_{2} x_{1} x_{3}\right)^{\beta} x_{2},
\end{array}\right. \\
& \left\{\begin{array}{l}
x_{1} \rightarrow\left(x_{3} x_{1} x_{2}\right)^{\tau} x_{3} x_{1} \\
x_{2} \rightarrow x_{1} x_{2} \\
x_{4} \rightarrow x_{2} x_{3}\left(x_{1} x_{2} x_{3}\right)^{\alpha-\beta-\tau-1}\left(x_{3} x_{1} x_{2}\right)^{\tau} x_{3} x_{1}\left(x_{1} x_{2} x_{3}\right)^{\beta} x_{1} x_{2},
\end{array}\right.
\end{aligned}
$$

where $\beta, \lambda, \sigma, \tau$ are natural parameters, with $\beta \leq \alpha, \sigma+\beta \leq \alpha, \tau+\beta+1 \leq \alpha$.

Proof. The equation $\mathbf{1 0}^{*}$ can be reduced by the transformation

$$
x_{2} \rightarrow x_{1} x_{2}
$$

to the equation $E_{1}$ :

$$
x_{3}^{\alpha+1} x_{1} x_{2}=x_{2} x_{1} x_{4} \quad \text { with } 0<\partial\left(x_{2}\right)<\partial\left(x_{3}^{\alpha+1}\right) .
$$

The equation $E_{1}$ can be reduced by the transformation

$$
\left\{\begin{array}{l}
x_{2} \rightarrow\left(x_{2} x_{3}\right)^{\beta} x_{2}, \\
x_{3} \rightarrow x_{2} x_{3}
\end{array}\right.
$$

where $\beta$ is a natural parameter with $\beta \leq \alpha$, to the equation $E_{2}$ :

$$
x_{3}\left(x_{2} x_{3}\right)^{\alpha-\beta} x_{1}\left(x_{2} x_{3}\right)^{\beta} x_{2}=x_{1} x_{4} \quad \text { with } \partial\left(x_{3}\right)>0 .
$$

According to Proposition 27, the equation $E_{2}$ is reduced by the parametric transformation

$$
x_{1} \rightarrow\left(x_{3}\left(x_{2} x_{3}\right)^{\alpha-\beta}\right)^{\lambda} x_{1}
$$


where $\lambda$ is a natural parameter, to the equation $E_{3}$ :

$$
E_{2} \quad \text { with } \partial\left(x_{1}\right)<\partial\left(x_{3}\left(x_{2} x_{3}\right)^{\alpha-\beta}\right) .
$$

The equation $E_{3}$ can be divided into the collection of equations

(j) $E_{3}$ with $\partial\left(x_{3} x_{2}\right)^{\sigma} \leq \partial\left(x_{1}\right)<\partial\left(\left(x_{3} x_{2}\right)^{\sigma} x_{3}\right), \sigma \leq \alpha-\beta$,

(jj) $E_{3}$ with $\partial\left(\left(x_{3} x_{2}\right)^{\tau} x_{3}\right) \leq \partial\left(x_{1}\right)<\partial\left(\left(x_{3} x_{2}\right)^{\tau+1} x_{3}\right), \tau \leq \alpha-\beta-1$.

The equation $(\mathrm{j})$ can be reduced by the transformation

$$
\left\{\begin{array}{l}
x_{1} \rightarrow\left(x_{1} x_{3} x_{2}\right)^{\sigma} x_{1}, \\
x_{3} \rightarrow x_{1} x_{3}
\end{array}\right.
$$

to the equation

$$
x_{3}\left(x_{2} x_{1} x_{3}\right)^{\alpha-\beta-\sigma}\left(x_{1} x_{3} x_{2}\right)^{\sigma} x_{1}\left(x_{2} x_{1} x_{3}\right)^{\beta} x_{2}=x_{4} .
$$

The equation (jj) can be reduced by the transformation

$$
\left\{\begin{array}{l}
x_{1} \rightarrow\left(x_{3} x_{1} x_{2}\right)^{\tau} x_{3} x_{1}, \\
x_{2} \rightarrow x_{1} x_{2}
\end{array}\right.
$$

to the equation

$$
x_{2} x_{3}\left(x_{1} x_{2} x_{3}\right)^{\alpha-\beta-\tau-1}\left(x_{3} x_{1} x_{2}\right)^{\tau} x_{3} x_{1}\left(x_{1} x_{2} x_{3}\right)^{\beta} x_{1} x_{2}=x_{4} .
$$

Proposition 10. The general solution of the equation

10

$$
x_{1} x_{3}^{\alpha+1} x_{2}=x_{2} x_{1} x_{4},
$$

where $\alpha$ is a natural parameter, is described by the transformation

$$
\left\{\begin{array}{l}
x_{1} \rightarrow^{\mathbf{F i}}\left(x_{1} x_{3}^{\alpha+1}, x_{2}\right)^{\mu} x_{1}, \\
x_{2} \rightarrow^{\mathbf{F i}}\left(x_{2}, x_{1} x_{3}^{\alpha}\right)^{\mu \mid} x_{2},
\end{array}\right.
$$

followed by one of the three transformations

$$
\left\{\begin{array} { l } 
{ x _ { 1 } \rightarrow 1 , } \\
{ x _ { 3 } \rightarrow 1 , } \\
{ x _ { 4 } \rightarrow 1 , }
\end{array} \quad \left\{\begin{array}{l}
x_{2} \rightarrow 1, \\
x_{4} \rightarrow x_{3}^{\alpha+1},
\end{array} \quad\left\langle\mathbf{1 0}^{*}\right\rangle\right.\right.
$$

Proof. This follows directly from Propositions 9 and $10^{*}$.

$$
\text { 5. The FUnCtion }{ }^{\mathbf{T h}}\left(x_{1}, x_{2}, x_{3}\right)_{i}^{\lambda_{1}, \ldots, \lambda_{2 s}}
$$

Theorem ${ }^{\text {Th }} \mathbf{1}$. The following identities hold for $s \geq 0$ :

$$
\begin{gathered}
\mathbf{T h}_{\left(x_{1}, x_{2}, x_{3}\right)_{1}^{\lambda_{1}, \ldots, \lambda_{2 s+2}}} \mathbf{\lambda}^{\mathbf{T h}}\left(\left(x_{1} x_{2} x_{3}\right)^{\lambda_{2 s+1}} x_{1},\left(x_{2} x_{3} x_{1}\left(x_{1} x_{2} x_{3}\right)^{\lambda_{2 s+1}} x_{1}\right)^{\lambda_{2 s+2}} x_{2},\right. \\
\left.x_{1} x_{2} x_{3}\right)_{1}^{\lambda_{1}, \ldots, \lambda_{2 s}}\left(x_{1} x_{2} x_{3}\right)^{\lambda_{2 s+1}} ; \\
\mathbf{T h}_{\left(x_{1}, x_{2}, x_{3}\right)_{2}^{\lambda_{1}, \ldots, \lambda_{2 s+2}}}={ }^{\mathbf{T h}}\left(\left(x_{1} x_{2} x_{3}\right)^{\lambda_{2 s+1}} x_{1},\left(x_{2} x_{3} x_{1}\left(x_{1} x_{2} x_{3}\right)^{\lambda_{2 s+1}} x_{1}\right)^{\lambda_{2 s+2}} x_{2},\right. \\
\left.x_{1} x_{2} x_{3}\right)_{2}^{\lambda_{1}, \ldots, \lambda_{2 s}}\left(x_{2} x_{3} x_{1}\left(x_{1} x_{2} x_{3}\right)^{\lambda_{2 s+1}} x_{1}\right)^{\lambda_{2 s+2}} ;
\end{gathered}
$$




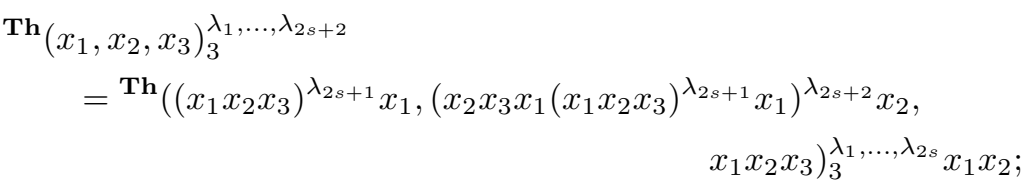

$$
\begin{array}{r}
\text { Oc }\left(x_{1}, x_{2}, x_{3}\right)^{\lambda_{1}, \ldots, \lambda_{2 s+2}} \\
=\left(x_{3} x_{1} x_{2}\right)^{\lambda_{2 s+1} \mathbf{O c}}\left(\left(x_{1} x_{2} x_{3}\right)^{\lambda_{2 s+1}} x_{1},\left(x_{2} x_{3} x_{1}\left(x_{1} x_{2} x_{3}\right)^{\lambda_{2 s+1}} x_{1}\right)^{\lambda_{2 s+2}}\right. \\
\left.x_{2}, x_{1} x_{2} x_{3}\right)^{\lambda_{1}, \ldots, \lambda_{2 s}} .
\end{array}
$$

Proof. We argue by a joint induction on $s$.

Third identity. By definition,

$$
\begin{aligned}
& \mathbf{T h} \\
& \left(x_{1}, x_{2}, x_{3}\right)_{3}^{\lambda_{1}, \ldots, \lambda_{2 s+2}} \\
& ={ }^{\mathbf{T h}}\left(x_{1}, x_{2}, x_{3}\right)_{1}^{\lambda_{3}, \ldots, \lambda_{2 s+2}} x_{1}^{\mathbf{T h}}\left(x_{1}, x_{2}, x_{3}\right)_{2}^{\lambda_{3}, \ldots, \lambda_{2 s+2}} x_{2}{ }^{\mathbf{T h}}\left(x_{1}, x_{2}, x_{3}\right)_{3}^{\lambda_{3}, \ldots, \lambda_{2 s+2}} .
\end{aligned}
$$

According to the induction proposition, it is equal to

$$
\begin{aligned}
& \mathbf{T h}\left(\left(x_{1} x_{2} x_{3}\right)^{\lambda_{2 s+1}} x_{1},\left(x_{2} x_{3} x_{1}\left(x_{1} x_{2} x_{3}\right)^{\lambda_{2 s+1}} x_{1}\right)^{\lambda_{2 s+2}} x_{2},\right. \\
& \left.x_{1} x_{2} x_{3}\right)_{1}^{\lambda_{3}, \ldots, \lambda_{2 s}}\left(x_{1} x_{2} x_{3}\right)^{\lambda_{2 s+1}} x_{1} \\
& .{ }^{\mathbf{T h}}\left(\left(x_{1} x_{2} x_{3}\right)^{\lambda_{2 s+1}} x_{1},\left(x_{2} x_{3} x_{1}\left(x_{1} x_{2} x_{3}\right)^{\lambda_{2 s+1}} x_{1}\right)^{\lambda_{2 s+2}} x_{2}, x_{1} x_{2} x_{3}\right)_{2}^{\lambda_{3}, \ldots, \lambda_{2 s}} \\
& \cdot\left(x_{2} x_{3} x_{1}\left(x_{1} x_{2} x_{3}\right)^{\lambda_{2 s+1}} x_{1}\right)^{\lambda_{2 s+2}} x_{2} \\
& .{ }^{\mathbf{T h}}\left(\left(x_{1} x_{2} x_{3}\right)^{\lambda_{2 s+1}} x_{1},\left(x_{2} x_{3} x_{1}\left(x_{1} x_{2} x_{3}\right)^{\lambda_{2 s+1}} x_{1}\right)^{\lambda_{2 s+2}} x_{2}, x_{1} x_{2} x_{3}\right)_{3}^{\lambda_{3}, \ldots, \lambda_{2 s}} x_{1} x_{2} .
\end{aligned}
$$

This is equal by definition to

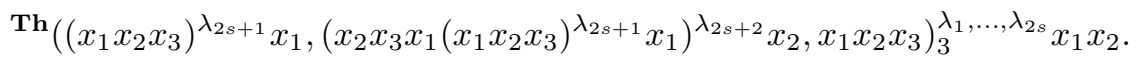

First identity. By definition,

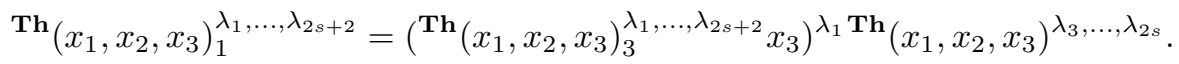

According to the induction proposition and the third identity this is equal to

$$
\begin{gathered}
\left({ }^{\mathbf{T h}}\left(\left(x_{1} x_{2} x_{3}\right)^{\lambda_{2 s+1}} x_{1},\left(x_{2} x_{3} x_{1}\left(x_{1} x_{2} x_{3}\right)^{\lambda_{2 s+1}} x_{1}\right)^{\lambda_{2 s+2}} x_{2}, x_{1} x_{2} x_{3}\right)_{3}^{\lambda_{1}, \ldots, \lambda_{2 s}} x_{1} x_{2} x_{3}\right)^{\lambda_{1}} \\
.{ }^{\mathbf{T h}}\left(\left(x_{1} x_{2} x_{3}\right)^{\lambda_{2 s+1}} x_{1},\left(x_{2} x_{3} x_{1}\left(x_{1} x_{2} x_{3}\right)^{\lambda_{2 s+1}} x_{1}\right)^{\lambda_{2 s+2}} x_{2}, x_{1} x_{2} x_{3}\right)_{1}^{\lambda_{3}, \ldots, \lambda_{2 s}} \\
\cdot\left(x_{1} x_{2} x_{3}\right)^{\lambda_{2 s+1}} \cdot
\end{gathered}
$$

This is equal by definition to

$$
\begin{array}{r}
\mathbf{T h} \\
\left(\left(x_{1} x_{2} x_{3}\right)^{\lambda_{2 s+1}} x_{1},\left(x_{2} x_{3} x_{1}\left(x_{1} x_{2} x_{3}\right)^{\lambda_{2 s+1}} x_{1}\right)^{\lambda_{2 s+2}} x_{2}, x_{1} x_{2} x_{3}\right)_{1}^{\lambda_{1}, \ldots, \lambda_{2 s}} \\
\cdot\left(x_{1} x_{2} x_{3}\right)^{\lambda_{2 s+1}} .
\end{array}
$$

Second identity. By definition

$$
\begin{aligned}
\left.\mathbf{T h}_{(} x_{1}, x_{2}, x_{3}\right)_{2}^{\lambda_{1}, \ldots, \lambda_{2 s+2}} & \\
= & \left({ }^{\mathbf{T h}}\left(x_{1}, x_{2}, x_{3}\right)_{2}^{\lambda_{3}, \ldots, \lambda_{2 s+2}} x_{2}{ }^{\mathbf{T h}}\left(x_{1}, x_{2}, x_{3}\right)_{3}^{\lambda_{3}, \ldots, \lambda_{2 s+2}} x_{3}\right. \\
& \left..{ }^{\mathbf{T h}}\left(x_{1}, x_{2}, x_{3}\right)_{1}^{\lambda_{3}, \ldots, \lambda_{2 s+2}} x_{1}{ }^{\mathbf{T h}}\left(x_{1}, x_{2}, x_{3}\right)_{1}^{\lambda_{1}, \ldots, \lambda_{2 s+2}} x_{1}\right)^{\lambda_{2}} \\
& .{ }^{\mathbf{T h}}\left(x_{1}, x_{2}, x_{3}\right)_{2}^{\lambda_{3}, \ldots, \lambda_{2 s+2}} .
\end{aligned}
$$


According to the induction proposition and the first identity, this is equal to

$$
\begin{gathered}
\left({ }^{\mathbf{T h}}\left(\left(x_{1} x_{2} x_{3}\right)^{\lambda_{2 s+1}} x_{1},\left(x_{2} x_{3} x_{1}\left(x_{1} x_{2} x_{3}\right)^{\lambda_{2 s+1}} x_{1}\right)^{\lambda_{2 s+2}} x_{2}, x_{1} x_{2} x_{3}\right)_{2}^{\lambda_{3}, \ldots, \lambda_{2 s}}\right. \\
\cdot\left(x_{2} x_{3} x_{1}\left(x_{1} x_{2} x_{3}\right)^{\lambda_{2 s+1}} x_{1}\right)^{\lambda_{2 s+2}} x_{2} \\
.{ }^{\mathbf{T h}}\left(\left(x_{1} x_{2} x_{3}\right)^{\lambda_{2 s+1}} x_{1},\left(x_{2} x_{3} x_{1}\left(x_{1} x_{2} x_{3}\right)^{\lambda_{2 s+1}} x_{1}\right)^{\lambda_{2 s+2}} x_{2}, x_{1} x_{2} x_{3}\right)_{3}^{\lambda_{3}, \ldots, \lambda_{2 s}} \\
\cdot x_{1} x_{2} x_{3} \\
.{ }^{\mathbf{T h}}\left(\left(x_{1} x_{2} x_{3}\right)^{\lambda_{2 s+1}} x_{1},\left(x_{2} x_{3} x_{1}\left(x_{1} x_{2} x_{3}\right)^{\lambda_{2 s+1}} x_{1}\right)^{\lambda_{2 s+2}} x_{2}, x_{1} x_{2} x_{3}\right)_{1}^{\lambda_{3}, \ldots, \lambda_{2 s}} \\
\cdot\left(x_{1} x_{2} x_{3}\right)^{\lambda_{2 s+1}} x_{1} \\
.{ }^{\mathbf{T h}}\left(\left(x_{1} x_{2} x_{3}\right)^{\lambda_{2 s+1}} x_{1},\left(x_{2} x_{3} x_{1}\left(x_{1} x_{2} x_{3}\right)^{\lambda_{2 s+1}} x_{1}\right)^{\lambda_{2 s+2}} x_{2}, x_{1} x_{2} x_{3}\right)_{1}^{\lambda_{1}, \ldots, \lambda_{2 s}} \\
\left.\cdot\left(x_{1} x_{2} x_{3}\right)^{\lambda_{2 s+1}} x_{1}\right)^{\lambda_{2}} \\
.{ }^{\mathbf{T h}}\left(\left(x_{1} x_{2} x_{3}\right)^{\lambda_{2 s+1}} x_{1},\left(x_{2} x_{3} x_{1}\left(x_{1} x_{2} x_{3}\right)^{\lambda_{2 s+1}} x_{1}\right)^{\lambda_{2 s+2}} x_{2}, x_{1} x_{2} x_{3}\right)_{2}^{\lambda_{3}, \ldots, \lambda_{2 s}} \\
\cdot\left(x_{2} x_{3} x_{1}\left(x_{1} x_{2} x_{3}\right)^{\lambda_{2 s+1}} x_{1}\right)^{\lambda_{2 s+2}} .
\end{gathered}
$$

This is equal by definition to

$$
\begin{gathered}
\left.\operatorname{Th}_{(}\left(x_{1} x_{2} x_{3}\right)^{\lambda_{2 s+1}} x_{1},\left(x_{2} x_{3} x_{1}\left(x_{1} x_{2} x_{3}\right)^{\lambda_{2 s+1}} x_{1}\right)^{\lambda_{2 s+2}} x_{2}, x_{1} x_{2} x_{3}\right)_{2}^{\lambda_{1}, \ldots, \lambda_{2 s}} \\
\cdot\left(x_{2} x_{3} x_{1}\left(x_{1} x_{2} x_{3}\right)^{\lambda_{2 s+1}} x_{1}\right)^{\lambda_{2 s+2}} .
\end{gathered}
$$

Fourth identity. By definition,

$$
\begin{aligned}
& \mathbf{O c}\left(\left(x_{1}, x_{2}, x_{3}\right)^{\lambda_{1}, \ldots, \lambda_{2 s+2}}\right. \\
& ={ }^{\mathbf{O c}}\left(x_{1}, x_{2}, x_{3}\right)^{\lambda_{3}, \ldots, \lambda_{2 s+2}}\left({ }^{\mathbf{T h}}\left(x_{1}, x_{2}, x_{3}\right)_{3}^{\lambda_{3}, \ldots, \lambda_{2 s+2}} x_{3}{ }^{\mathbf{T h}}\left(x_{1}, x_{2}, x_{3}\right)_{1}^{\lambda_{3}, \ldots, \lambda_{2 s+2}} x_{1}\right. \\
& \left.{ }^{\mathbf{T h}}\left(x_{1}, x_{2}, x_{3}\right)_{2}^{\lambda_{3}, \ldots, \lambda_{2 s+2}} x_{2}\right)^{\lambda_{1}} .
\end{aligned}
$$

According to the induction proposition and the first, second and third identities, this is equal to

$$
\begin{gathered}
\left(x_{3} x_{1} x_{2}\right)^{\lambda_{2 s+1} \mathbf{O c}}\left(\left(x_{1} x_{2} x_{3}\right)^{\lambda_{2 s+1}} x_{1},\left(x_{2} x_{3} x_{1}\left(x_{1} x_{2} x_{3}\right)^{\lambda_{2 s+1}} x_{1}\right)^{\lambda_{2 s+2}} x_{2}, x_{1} x_{2} x_{3}\right)^{\lambda_{3}, \ldots, \lambda_{2 s}} \\
\cdot\left({ }^{\mathbf{T h}}\left(\left(x_{1} x_{2} x_{3}\right)^{\lambda_{2 s+1}} x_{1},\left(x_{2} x_{3} x_{1}\left(x_{1} x_{2} x_{3}\right)^{\lambda_{2 s+1}} x_{1}\right)^{\lambda_{2 s+2}} x_{2}, x_{1} x_{2} x_{3}\right)_{3}^{\lambda_{3}, \ldots, \lambda_{2 s}} x_{1} x_{2} x_{3}\right. \\
\cdot{ }^{\mathbf{T h}}\left(\left(x_{1} x_{2} x_{3}\right)^{\lambda_{2 s+1}} x_{1},\left(x_{2} x_{3} x_{1}\left(x_{1} x_{2} x_{3}\right)^{\lambda_{2 s+1}} x_{1}\right)^{\lambda_{2 s+2}} x_{2}, x_{1} x_{2} x_{3}\right)_{1}^{\lambda_{3}, \ldots, \lambda_{2 s}} \\
\cdot\left(x_{1} x_{2} x_{3}\right)^{\lambda_{2 s+1}} x_{1} \\
\cdot{ }^{\mathbf{T h}}\left(\left(x_{1} x_{2} x_{3}\right)^{\lambda_{2 s+1}} x_{1},\left(x_{2} x_{3} x_{1}\left(x_{1} x_{2} x_{3}\right)^{\lambda_{2 s+1}} x_{1}\right)^{\lambda_{2 s+2}} x_{2}, x_{1} x_{2} x_{3}\right)_{2}^{\lambda_{3}, \ldots, \lambda_{s}} \\
\left.\cdot\left(x_{2} x_{3} x_{1}\left(x_{1} x_{2} x_{3}\right)^{\lambda_{2 s+1}} x_{1}\right)^{\lambda_{2 s+2}} x_{2}\right)^{\lambda_{1}} \cdot
\end{gathered}
$$

This is equal by definition to

$$
\begin{array}{r}
\left(x_{3} x_{1} x_{2}\right)^{\lambda_{2 s+1} \mathbf{O c}}\left(\left(x_{1} x_{2} x_{3}\right)^{\lambda_{2 s+1}} x_{1},\left(x_{2} x_{3} x_{1}\left(x_{1} x_{2} x_{3}\right)^{\lambda_{2 s+1}} x_{1}\right)^{\lambda_{2 s+2}} x_{2},\right. \\
\left.x_{1} x_{2} x_{3}\right)^{\lambda_{1}, \ldots, \lambda_{2 s}} .
\end{array}
$$

Consider the equation

$$
x_{1} x_{2} x_{3} x_{4}=x_{3} x_{1}^{2} x_{2}
$$


and the sequence of joint parametric transformations (13):

$$
\begin{aligned}
& \left\{\begin{array}{l}
x_{1} \rightarrow\left(x_{1} x_{2} x_{3}\right)^{\lambda_{1}} x_{1}, \\
x_{2} \rightarrow\left(x_{2} x_{3} x_{1}\left(x_{1} x_{2} x_{3}\right)^{\lambda_{1}} x_{1}\right)^{\lambda_{2}} x_{2}, \\
x_{3} \rightarrow x_{1} x_{2} x_{3}, \\
x_{4} \rightarrow x_{4}\left(x_{3} x_{1} x_{2}\right)^{\lambda_{1}} ;
\end{array}\right. \\
& \left\{\begin{array}{l}
x_{1} \rightarrow\left(x_{1} x_{2} x_{3}\right)^{\lambda_{3}} x_{1}, \\
x_{2} \rightarrow\left(x_{2} x_{3} x_{1}\left(x_{1} x_{2} x_{3}\right)^{\lambda_{3}} x_{1}\right)^{\lambda_{4}} x_{2}, \\
x_{3} \rightarrow x_{1} x_{2} x_{3} \\
x_{4} \rightarrow x_{4}\left(x_{3} x_{1} x_{2}\right)^{\lambda_{3}} ;
\end{array}\right. \\
& \left\{\begin{array}{l}
\ldots \ldots \ldots \\
x_{1} \rightarrow\left(x_{1} x_{2} x_{3}\right)^{\lambda_{2 s-1}} x_{1}, \\
x_{2} \rightarrow\left(x_{2} x_{3} x_{1}\left(x_{1} x_{2} x_{3}\right)^{\lambda_{2 s-1}} x_{1}\right)^{\lambda_{2 s}} x_{2}, \\
x_{3} \rightarrow x_{1} x_{2} x_{3}, \\
x_{4} \rightarrow x_{4}\left(x_{3} x_{1} x_{2}\right)^{\lambda_{2 s-1}} ;
\end{array}\right. \\
& \left\{\begin{array}{l}
x_{1} \rightarrow\left(x_{1} x_{2} x_{3}\right)^{\lambda_{2 s+1}} x_{1}, \\
x_{2} \rightarrow\left(x_{2} x_{3} x_{1}\left(x_{1} x_{2} x_{3}\right)^{\lambda_{2 s+1}} x_{1}\right)^{\lambda_{2 s+2}} x_{2}, \\
x_{3} \rightarrow x_{1} x_{2} x_{3}, \\
x_{4} \rightarrow x_{4}\left(x_{3} x_{1} x_{2}\right)^{\lambda_{2 s+1}} ;
\end{array}\right.
\end{aligned}
$$

Theorem ${ }^{\mathbf{T h}} \mathbf{2}$. For every natural $s$ the sequence of the $s$ joint parametric transformations (13) can be collected by the following common transformation:

$$
\left\{\begin{array}{l}
x_{1} \rightarrow{ }^{\mathbf{T h}}\left(x_{1}, x_{2}, x_{3}\right)_{1}^{\lambda_{1}, \ldots, \lambda_{2 s}} x_{1}, \\
x_{2} \rightarrow \mathbf{T h}\left(x_{1}, x_{2}, x_{3}\right)_{2}^{\lambda_{1}, \ldots, \lambda_{2 s}} x_{2}, \\
x_{3} \rightarrow \mathbf{T h}\left(x_{1}, x_{2}, x_{3}\right)_{3}^{\lambda_{1}, \ldots, \lambda_{2 s}} x_{3}, \\
x_{4} \rightarrow x_{4}{ }^{\mathbf{O c}}\left(x_{1}, x_{2}, x_{3}\right)^{\lambda_{1}, \ldots, \lambda_{2 s}}
\end{array}\right.
$$

Proof. Suppose the sequence of the first $s$ transformations can be collected by the common transformation (14), and let the $(s+1)$ st transformation be of the form $(13 . s+1)$. Then the sequence of the first $s+1$ transformations can be collected by the common transformation

$$
\left\{\begin{aligned}
x_{1} \rightarrow & \mathbf{T h} \\
( & \left.\left(x_{1} x_{2} x_{3}\right)^{\lambda_{2 s+1}} x_{1},\left(x_{2} x_{3} x_{1}\left(x_{1} x_{2} x_{3}\right)^{\lambda_{2 s+1}} x_{1}\right)^{\lambda_{2 s+2}} x_{2}, x_{1} x_{2} x_{3}\right)_{1}^{\lambda_{1}, \ldots, \lambda_{2 s}} \\
& \cdot\left(x_{1} x_{2} x_{3}\right)^{\lambda_{2 s+1}} x_{1}, \\
x_{2} \rightarrow \mathbf{T h} & \left(\left(x_{1} x_{2} x_{3}\right)^{\lambda_{2 s+1}} x_{1},\left(x_{2} x_{3} x_{1}\left(x_{1} x_{2} x_{3}\right)^{\lambda_{2 s+1}} x_{1}\right)^{\lambda_{2 s+2}} x_{2}, x_{1} x_{2} x_{3}\right)_{2}^{\lambda_{1}, \ldots, \lambda_{2 s}} \\
& \cdot\left(x_{2} x_{3} x_{1}\left(x_{1} x_{2} x_{3}\right)^{\lambda_{2 s+1}} x_{1}\right)^{\lambda_{2 s+2}} x_{2}, \\
x_{3} \rightarrow \mathbf{T h} & \left(\left(x_{1} x_{2} x_{3}\right)^{\lambda_{2 s+1}} x_{1},\left(x_{2} x_{3} x_{1}\left(x_{1} x_{2} x_{3}\right)^{\lambda_{2 s+1}} x_{1}\right)^{\lambda_{2 s+2}} x_{2}, x_{1} x_{2} x_{3}\right)_{3}^{\lambda_{1}, \ldots, \lambda_{2 s}} \\
& \cdot x_{1} x_{2} x_{3}, \\
x_{4} \rightarrow & x_{4}\left(x_{3} x_{1} x_{2}\right)^{\lambda_{2 s+1}} \\
& \quad \text { Oc }\left(\left(x_{1} x_{2} x_{3}\right)^{\lambda_{2 s+1}} x_{1},\left(x_{2} x_{3} x_{1}\left(x_{1} x_{2} x_{3}\right)^{\lambda_{2 s+1}} x_{1}\right)^{\lambda_{2 s+2}} x_{2}, x_{1} x_{2} x_{3}\right)^{\lambda_{1}, \ldots, \lambda_{2 s}} .
\end{aligned}\right.
$$


According to Theorem ${ }^{\mathbf{T h}} 1$ this transformation coincides with the transformation

$$
\left\{\begin{array}{l}
x_{1} \rightarrow \mathbf{T h}\left(x_{1}, x_{2}, x_{3}\right)_{1}^{\lambda_{1}, \ldots, \lambda_{2 s+2}} x_{1}, \\
x_{2} \rightarrow \mathbf{T h}\left(x_{1}, x_{2}, x_{3}\right)_{2}^{\lambda_{1}, \ldots, \lambda_{2 s+2}} x_{2}, \\
x_{3} \rightarrow \mathbf{T h}\left(x_{1}, x_{2}, x_{3}\right)_{3}^{\lambda_{1}, \ldots, \lambda_{2 s+2}} x_{3}, \\
x_{4} \rightarrow x_{4} \mathbf{O c}\left(x_{1}, x_{2}, x_{3}\right)^{\lambda_{1}, \ldots, \lambda_{2 s+2}} .
\end{array}\right.
$$

Proposition 11. The general solution of the equation

11

$$
x_{2} x_{1} x_{3} x_{4}=x_{3} x_{1}^{2}\left(x_{3} x_{1}\right)^{\alpha} x_{2}
$$

with $\partial\left(x_{3}\right) \leq \partial\left(x_{2}\right)<\partial\left(x_{3} x_{1}^{2}\left(x_{3} x_{1}\right)^{\alpha}\right)$, where $\alpha$ is a natural parameter, is described by the transformations

$\mathrm{t}\left\{\begin{array}{l}x_{1} \rightarrow x_{1}^{\eta+\theta} \\ x_{2} \rightarrow x_{3} x_{1}^{\theta} \\ x_{4} \rightarrow x_{4}\left(x_{1}^{\eta+\theta} x_{3}\right)^{\alpha} x_{1}^{\theta}\end{array}\right.$

$$
\mathrm{tt}\left\{\begin{array}{l}
x_{2} \rightarrow x_{3} x_{1}\left(x_{1} x_{3}\right)^{\gamma} x_{2} \\
x_{1} \rightarrow x_{2} x_{1} \\
x_{4} \rightarrow\left(x_{1} x_{3} x_{2}\right)^{\alpha-\gamma} x_{1} x_{2}\left(x_{1} x_{3} x_{2}\right)^{\gamma}
\end{array}\right.
$$

$\mathrm{ttt}\left\{\begin{array}{l}x_{2} \rightarrow x_{3} x_{1}\left(x_{1} x_{3}\right)^{\delta} x_{1} x_{2}, \\ x_{3} \rightarrow x_{2} x_{3}, \\ x_{4} \rightarrow\left(x_{3} x_{1} x_{2}\right)^{\alpha-\delta-1} x_{3} x_{1}^{2} x_{2}\left(x_{3} x_{1} x_{2}\right)^{\delta},\end{array}\right.$

$\langle\mathbf{2}\rangle$

where $\eta, \theta, \gamma, \delta$ are natural parameters with $\gamma \leq \alpha$ and $\delta<\alpha$.

Proof. It is easy to see that $\mathbf{1 1}$ can be divided into the following collection of equations:

(j) 11 with $\partial\left(x_{3}\right) \leq \partial\left(x_{2}\right)<\partial\left(x_{3} x_{1}\right)$,

(jj) 11 with $\partial\left(x_{3} x_{1}\left(x_{1} x_{3}\right)^{\gamma}\right) \leq \partial\left(x_{2}\right)<\partial\left(x_{3} x_{1}\left(x_{1} x_{3}\right)^{\gamma} x_{1}\right)$,

(jjj) 11 with $\partial\left(x_{3} x_{1}\left(x_{1} x_{3}\right)^{\delta} x_{1}\right) \leq \partial\left(x_{2}\right)<\partial\left(x_{3} x_{1}\left(x_{1} x_{3}\right)^{\delta+1}\right)$, where $\gamma, \delta$ are natural parameters with $\gamma \leq \alpha$ and $\delta<\alpha$.

It is obvious that $(\mathrm{j})$ is reduced by the parametric transformation

$$
\left\{\begin{array}{l}
x_{2} \rightarrow x_{3} x_{2} \\
x_{1} \rightarrow x_{2} x_{1} \\
x_{4} \rightarrow x_{4} x_{2}\left(x_{1} x_{3} x_{2}\right)^{\alpha}
\end{array}\right.
$$

to the equation $x_{2} x_{1} x_{3} x_{4}=x_{1} x_{2} x_{1} x_{3}$ with $\partial\left(x_{2}\right)<\partial\left(x_{1}\right)$. The last equation falls into the system

$$
\left\{\begin{array}{l}
x_{2} x_{1}=x_{1} x_{2} \\
x_{3} x_{4}=x_{1} x_{3}
\end{array}\right.
$$

with $\partial\left(x_{2}\right)<\partial\left(x_{1}\right)$. By Proposition 1 this system is reduced by the parametric transformation

$$
\left\{\begin{array}{l}
x_{1}=x_{1}^{\eta} \\
x_{2}=x_{1}^{\theta}
\end{array}\right.
$$

to the equation $x_{3} x_{4}=x_{1}^{\eta} x_{3}$ with $\theta>\eta$, that is, to the equation $\mathbf{5}$.

The equation $(\mathrm{jj})$ is reduced by the parametric transformation tt to the equation 2. 
The equation (jjj) is reduced by the parametric transformation ttt to the equation 2.

Proposition 12*. The parametric equation

$12^{*}$

$$
x_{2} x_{1} x_{3} x_{4}=x_{3} x_{1}^{2}\left(x_{3} x_{1}\right)^{\alpha} x_{2}
$$

with $\partial\left(x_{2}\right)<\partial\left(x_{3}\right)$, where $\alpha$ is a natural parameter, is reduced by the transformation

$$
\left\{\begin{array}{l}
x_{3} \rightarrow x_{2} x_{3} \\
x_{4} \rightarrow x_{4}\left(x_{3} x_{1} x_{2}\right)^{\alpha}
\end{array}\right.
$$

to the parametric equation $\mathbf{1 2 .}$

Proof. This is obvious.

Proposition 12. The general solution of the equation

12

$$
x_{1} x_{2} x_{3} x_{4}=x_{3} x_{1}^{2} x_{2} \quad \text { with } \partial\left(x_{3}\right)>0
$$

is described by the transformation

$$
\begin{gathered}
\mathrm{T}\left\{\begin{array}{l}
x_{1} \rightarrow{ }^{\mathbf{T h}}\left(x_{1}, x_{2}, x_{3}\right)_{1}^{\xi} x_{1}, \\
x_{2} \rightarrow \mathbf{T h}\left(x_{1}, x_{2}, x_{3}\right)_{2}^{\xi} x_{2}, \\
x_{3} \rightarrow \mathbf{T h}\left(x_{1}, x_{2}, x_{3}\right)_{3}^{\xi} x_{3}, \\
x_{4} \rightarrow x_{4}{ }^{\mathbf{O c}}\left(x_{1}, x_{2}, x_{3}\right)^{\xi},
\end{array}\right. \\
\mathrm{t}\left\{\begin{array}{l}
x_{1} \rightarrow\left(x_{1} x_{3}\right)^{\sigma} x_{1}, \\
x_{2} \rightarrow\left(x_{3} x_{1}^{2}\left(x_{3} x_{1}\right)^{\sigma}\right)^{\rho} x_{2}, \\
x_{3} \rightarrow x_{1} x_{3} \\
x_{4} \rightarrow x_{4},
\end{array}\right.
\end{gathered}
$$

where $\xi$ is a variable whose values are even sequences of natural parameters, and $\sigma, \rho$ are natural parameters.

Proof. It is easy to apply the transformation $\mathrm{Tt}\langle\mathbf{1 1}\rangle$ to the equation $\mathbf{1 2}$ and to verify, by using the definition of the functions $\mathbf{T h}^{\mathbf{T h}}\left(x_{1}, x_{2}, x_{3}\right)_{i}^{\lambda_{1}, \ldots, \lambda_{2 s}}$, that it is a parametric solution of $\mathbf{1 2}$.

According to Proposition 8 the equation $\mathbf{1 2}$ is reduced by the parametric transformation $x_{1} \rightarrow x_{3}^{\sigma} x_{1}$, where $\sigma$ is natural parameter, to the equation $E_{1}$ :

$$
x_{1} x_{2} x_{3} x_{4}=x_{3} x_{1} x_{3}^{\sigma} x_{1} x_{2} \quad \text { with } \partial\left(x_{1}\right) \leq \partial\left(x_{3}\right) .
$$

According to condition $\partial\left(x_{1}\right)<\partial\left(x_{3}\right)$ the equation $E_{1}$ is reduced by the parametric transformation $x_{3} \rightarrow x_{1} x_{3}$ to the equation $E_{2}$ :

$$
x_{2} x_{1} x_{3} x_{4}=x_{3} x_{1}\left(x_{1} x_{3}\right)^{\sigma} x_{1} x_{2} .
$$

According to Proposition 8 the equation $E_{2}$ is reduced by the parametric transformation

$$
x_{2} \rightarrow\left(x_{3} x_{1}\left(x_{1} x_{3}\right)^{\sigma} x_{1}\right)^{\rho} x_{2},
$$

where $\rho$ is a natural parameter, to the equation $E_{3}$ :

$$
x_{2} x_{1} x_{3} x_{4}=x_{3} x_{1}^{2}\left(x_{3} x_{1}\right)^{\sigma} x_{2} \quad \text { with } \partial\left(x_{2}\right)<\partial\left(x_{3} x_{1}^{2}\left(x_{3} x_{1}\right)^{\sigma}\right) .
$$

It is easy to see that $E_{3}$ can be divided into the following collection of equations: 
(j) $E_{3}$ with $\partial\left(x_{3}\right) \leq \partial\left(x_{2}\right)$,

(jj) $E_{3}$ with $\partial\left(x_{3}\right)>\partial\left(x_{2}\right)$.

The equation (j) is equation 11.

According to Proposition $12^{*}$ the equation (jj) is reduced by the transformation

$$
\left\{\begin{array}{l}
x_{3} \rightarrow x_{2} x_{3}, \\
x_{4} \rightarrow x_{4}\left(x_{3} x_{1} x_{2}\right)^{\sigma}
\end{array}\right.
$$

to the equation $\mathbf{1 2 .}$

The sequence of transformations

$$
\begin{aligned}
& x_{1} \rightarrow x_{3}^{\sigma} x_{1}, \\
& x_{3} \rightarrow x_{1} x_{3}, \\
& x_{2} \rightarrow\left(x_{3} x_{1}\left(x_{1} x_{3}\right)^{\sigma} x_{1}\right)^{\rho} x_{2}, \\
& x_{3} \rightarrow x_{2} x_{3}, \\
& x_{4} \rightarrow x_{4}\left(x_{3} x_{1} x_{2}\right)^{\alpha}
\end{aligned}
$$

can be collected by the following common transformation $r$ :

$$
\left\{\begin{array}{l}
x_{1} \rightarrow\left(x_{1} x_{2} x_{3}\right)^{\sigma} x_{1} \\
x_{2} \rightarrow\left(x_{2} x_{3} x_{1}\left(x_{1} x_{2} x_{3}\right)^{\sigma} x_{1}\right)^{\rho} x_{2} \\
x_{3} \rightarrow x_{1} x_{2} x_{3} \\
x_{4} \rightarrow x_{4}\left(x_{3} x_{1} x_{2}\right)^{\alpha} .
\end{array}\right.
$$

Using Theorem ${ }^{\mathbf{T h}} 2$, one can see that transformation $\mathrm{rT}$ can be obtained from $\mathrm{T}$ by replacing the parameter $\xi$ by the sequence $\sigma, \rho, \xi$.

\section{Supplement}

Proposition 13. The general solution of the equation

13

$$
x_{1} x_{2} x_{3} x_{4}=x_{3} x_{2}\left(x_{2}\left(x_{3} x_{2}\right)^{\lambda+1}\right)^{\kappa+1} x_{1}
$$

with $\partial\left(x_{1}\right) \leq \partial\left(x_{3} x_{2}\left(x_{2}\left(x_{3} x_{2}\right)^{\lambda+1}\right)^{\kappa+1}\right)$, where $\lambda$ and $\kappa$ are natural parameters, is described by the transformations

$$
\begin{gathered}
\mathrm{t} 1 \quad \begin{array}{l}
x_{3} \rightarrow x_{1} x_{3}, \\
x_{4} \rightarrow x_{4} x_{3}\left(x_{2} x_{1} x_{3}\right)^{\lambda} x_{2}\left(\left(x_{2} x_{1} x_{3}\right)^{\lambda+1} x_{2}\right)^{\kappa} x_{1},
\end{array} \\
\mathrm{t} 2 \quad\left\{\begin{array}{l}
x_{1} \rightarrow x_{3} x_{1}^{\eta}, \\
x_{2} \rightarrow x_{1}^{\eta+\theta} \\
x_{4} \rightarrow x_{4}\left(x_{1}^{\eta+\theta} x_{3}\right)^{\lambda} x_{1}^{\eta+\theta}\left(\left(x_{1}^{\eta+\theta} x_{3}\right)^{\lambda+1} x_{1}^{\eta+\theta}\right)^{\kappa} x_{3} x_{1}^{\eta},
\end{array}\right. \\
\mathrm{t} 3 \quad\langle\mathbf{5}\rangle, \\
\left\{\begin{array}{c}
x_{1} \rightarrow x_{3} x_{1} x_{2}\left(x_{1} x_{2}\left(x_{3} x_{1} x_{2}\right)^{\lambda+1}\right)^{\tau}\left(x_{1} x_{2} x_{3}\right)^{\sigma} x_{1}, \\
x_{4} x_{2},
\end{array}\right. \\
\quad \cdot x_{2}\left(x_{3} x_{1} x_{2}\right)^{\lambda-\sigma}\left(x_{1} x_{2}\left(x_{3} x_{1} x_{2} x_{3} x_{1} x_{2}\right)^{\lambda+1}\right)^{\tau+1}\left(x_{1} x_{2} x_{3}\right)^{\sigma} x_{1},
\end{gathered}
$$




$$
\mathrm{t} 4\left\{\begin{aligned}
x_{1} & \rightarrow x_{3} x_{1}^{\eta+\theta}\left(\left(x_{1}^{\eta+\theta} x_{3}\right)^{\lambda+1} x_{1}^{\eta+\theta}\right)^{\tau}\left(x_{1}^{\eta+\theta} x_{3}\right)^{\lambda+1} x_{1}^{\eta}, \\
x_{2} \rightarrow & x_{1}^{\eta+\theta} \\
x_{4} \rightarrow & x_{4} x_{1}^{\eta+\theta}\left(x_{3} x_{1}^{\eta+\theta}\right)^{\lambda}\left(\left(x_{1}^{\eta+\theta} x_{3}\right)^{\lambda+1} x_{1}^{\eta+\theta}\right)^{\kappa-\tau-1} x_{3} \\
& \left.\quad x_{1}^{\eta+\theta}\left(x_{1}^{\eta+\theta} x_{3}\right)^{\lambda+1} x_{1}^{\eta+\theta}\right)^{\tau}\left(x_{1}^{\eta+\theta} x_{3}\right)^{\lambda+1} x_{1}^{\eta},
\end{aligned}\right.
$$

$\mathrm{t} 5\left\{\begin{aligned} x_{1} \rightarrow & x_{1} x_{3}\left(x_{2} x_{1}\right)^{\beta+1} x_{2}\left(\left(\left(x_{2} x_{1}\right)^{\beta+1} x_{2} x_{1} x_{3}\right)^{\lambda+1}\left(x_{2} x_{1}\right)^{\beta+1} x_{2}\right)^{\kappa} \\ & \cdot\left(\left(x_{2} x_{1}\right)^{\beta+1} x_{2} x_{1} x_{3}\right)^{\lambda+1} x_{2} x_{1}, \\ x_{2} \rightarrow & \left(x_{2} x_{1}\right)^{\beta+1} x_{2}, \\ x_{3} \rightarrow & x_{1} x_{3} \\ x_{4} \rightarrow & \left(x_{2} x_{1}\right)^{\beta} x_{2}\left(\left(\left(x_{2} x_{1}\right)^{\beta+1} x_{2} x_{1} x_{3}\right)^{\lambda+1}\left(x_{2} x_{1}\right)^{\beta+1} x_{2}\right)^{\kappa} \\ & \cdot\left(\left(x_{2} x_{1}\right)^{\beta+1} x_{2} x_{1} x_{3}\right)^{\lambda+1} x_{2} x_{1},\end{aligned}\right.$

$\langle\mathbf{2}\rangle$,

$$
\mathrm{t} 6\left\{\begin{aligned}
x_{1} \rightarrow & x_{3}\left(x_{2} x_{3} x_{1}\right)^{\beta+1} x_{2}\left(\left(\left(x_{2} x_{3} x_{1}\right)^{\beta+1} x_{2} x_{3}\right)^{\lambda+1}\left(x_{2} x_{3} x_{1}\right)^{\beta+1} x_{2}\right)^{\kappa} \\
& \cdot\left(\left(x_{2} x_{3} x_{1}\right)^{\beta+1} x_{2} x_{3}\right)^{\lambda+1} x_{2} x_{3} x_{1}, \\
x_{2} \rightarrow & \left(x_{2} x_{3} x_{1}\right)^{\beta+1} x_{2}, \\
x_{4} \rightarrow & \left(x_{2} x_{3} x_{1}\right)^{\beta} x_{2}\left(\left(\left(x_{2} x_{3} x_{1}\right)^{\beta+1} x_{2} x_{3}\right)^{\lambda+1}\left(x_{2} x_{3} x_{1}\right)^{\beta+1} x_{2}\right)^{\kappa} \\
& \cdot\left(\left(x_{2} x_{3} x_{1}\right)^{\beta+1} x_{2} x_{3}\right)^{\lambda+1} x_{2} x_{3} x_{1},
\end{aligned}\right.
$$

$\langle\mathbf{2}\rangle$,

$\mathrm{t} 7\left\{\begin{aligned} x_{1} & \rightarrow x_{1} x_{3} x_{2}\left(\left(x_{2} x_{1} x_{3}\right)^{\lambda+1} x_{2}\right)^{\tau}\left(x_{2} x_{1} x_{3}\right)^{\sigma} x_{2} x_{1}, \\ x_{3} \rightarrow & x_{1} x_{3} \\ x_{4} \rightarrow & x_{3} x_{2}\left(x_{1} x_{3} x_{2}\right)^{\lambda-\sigma-1}\left(\left(x_{2} x_{1} x_{3}\right)^{\lambda+1} x_{2}\right)^{\kappa-\tau} x_{1} x_{3} x_{2} \\ & \cdot\left(\left(x_{2} x_{1} x_{3}\right)^{\lambda+1} x_{2}\right)^{\tau}\left(x_{2} x_{1} x_{3}\right)^{\sigma} x_{2} x_{1},\end{aligned}\right.$

$\mathrm{t} 8\left\{\begin{aligned} x_{1} & \rightarrow x_{1} x_{3} x_{2}\left(\left(x_{2} x_{1} x_{3}\right)^{\lambda+1} x_{2}\right)^{\tau}\left(x_{2} x_{1} x_{3}\right)^{\lambda} x_{2} x_{1}, \\ x_{3} \rightarrow & x_{1} x_{3}, \\ x_{4} \rightarrow & x_{4} x_{3}\left(x_{2} x_{1} x_{3}\right)^{\lambda} x_{2}\left(\left(x_{2} x_{1} x_{3}\right)^{\lambda+1} x_{2}\right)^{\kappa-\tau-1} x_{1} x_{3} x_{2} \\ & \cdot\left(\left(x_{3} \rightarrow x_{1} x_{3} x_{2} x_{1} x_{3}\right)^{\lambda+1} x_{2}\right)^{\tau}\left(x_{2} x_{1} x_{3}\right)^{\lambda} x_{2} x_{1},\end{aligned}\right.$

$\langle\mathbf{1 2}\rangle$,

$$
\mathrm{t} 9\left\{\begin{array}{l}
x_{1} \rightarrow x_{1} x_{3} x_{2}\left(\left(x_{2} x_{1} x_{3}\right)^{\lambda+1} x_{2}\right)^{\kappa}\left(x_{2} x_{1} x_{3}\right)^{\lambda} x_{2} x_{1}, \\
x_{3} \rightarrow x_{1} x_{3} \\
x_{4} \rightarrow x_{3} x_{2}\left(\left(x_{2} x_{1} x_{3}\right)^{\lambda+1} x_{2}\right)^{\kappa}\left(x_{2} x_{1} x_{3}\right)^{\lambda} x_{2} x_{1}
\end{array}\right.
$$

$\langle\mathbf{2}\rangle$,

where $\eta, \theta, \sigma, \tau, \beta$ are natural parameters.

Proof. The equation $\mathbf{1 3}$ can be divided into the collection of equations

(j) 13 with $\partial\left(x_{1}\right)<\partial\left(x_{3}\right)$;

(jj) 13 with $\partial\left(x_{3}\right) \leq \partial\left(x_{1}\right) \leq \partial\left(x_{3} x_{2}\right)$; 
(jjj) 13 with

$$
\begin{aligned}
& \partial\left(x_{3} x_{2}\left(\left(x_{2} x_{3}\right)^{\lambda+1} x_{2}\right)^{\tau}\left({ }_{2} x_{3}\right)^{\sigma}\right) \leq \partial\left(x_{1}\right) \\
& \leq \partial\left(x_{3} x_{2}\left(\left(x_{2} x_{3}\right)^{\lambda+1} x_{2}\right)^{\tau}\left(x_{2} x_{3}\right)^{\sigma} x_{2}\right), \quad \tau \leq \kappa, \sigma \leq \lambda+1 ;
\end{aligned}
$$

(jjjj) 13 with

$$
\begin{aligned}
& \partial\left(x_{3} x_{2}\left(\left(x_{2} x_{3}\right)^{\lambda+1} x_{2}\right)^{\tau}\left(x_{2} x_{3}\right)^{\sigma} x_{2}\right) \leq \partial\left(x_{1}\right) \\
&<\partial\left(x_{3} x_{2}\left(\left(x_{2} x_{3}\right)^{\lambda+1} x_{2}\right)^{\tau}\left(x_{2} x_{3}\right)^{\sigma+1}\right), \quad \tau \leq \kappa, \sigma \leq \lambda .
\end{aligned}
$$

The equation $(\mathrm{j})$ can be reduced by the transformation

$$
\begin{aligned}
& x_{3} \rightarrow x_{1} x_{3}, \\
& x_{4} \rightarrow x_{4} x_{3}\left(x_{2} x_{1} x_{3}\right)^{\lambda} x_{2}\left(\left(x_{2}\left(x_{1} x_{3} x_{2}\right)^{\lambda+1}\right)^{\kappa} x_{1}\right.
\end{aligned}
$$

to the equation $x_{2} x_{1} x_{3} x_{4}=x_{3} x_{2}^{2} x_{1}$ with $\partial\left(x_{3}\right)>0$, that is, to the equation $\mathbf{1 2}$.

The equation (jj) can be reduced by the transformation

$$
\begin{aligned}
& x_{1} \rightarrow x_{3} x_{1}, \\
& x_{2} \rightarrow x_{1} x_{2}, \\
&\left\{\begin{array}{l}
x_{1} \rightarrow x_{1}^{\eta}, \\
x_{2} \rightarrow x_{1}^{\theta},
\end{array}\right. \\
& x_{4} \rightarrow x_{4}\left(x_{1}^{\eta+\theta} x_{3}\right)^{\lambda} x_{1}^{\eta+\theta}\left(\left(x_{1}^{\eta+\theta} x_{3}\right)^{\lambda+1} x_{1}^{\eta+\theta}\right)^{\kappa} x_{3} x_{1}^{\eta}
\end{aligned}
$$

to the equation $x_{3} x_{4}=x_{1}^{\theta} x_{3}$, that is, to the equation $\mathbf{5}$.

The equation (jjj) can be reduced by the transformation

$$
\begin{aligned}
& x_{1} \rightarrow x_{3} x_{2}\left(\left(x_{2} x_{3}\right)^{\lambda+1} x_{2}\right)^{\tau}\left(x_{2} x_{3}\right)^{\sigma} x_{1}, \\
& x_{2} \rightarrow x_{1} x_{2}
\end{aligned}
$$

to the equation $E$ :

$$
\begin{gathered}
x_{1} x_{2} x_{3} x_{4}=x_{2}\left(x_{3} x_{1} x_{2}\right)^{\lambda+1-\sigma}\left(\left(x_{1} x_{2} x_{3}\right)^{\lambda+1} x_{1} x_{2}\right)^{\kappa-\tau} x_{3} x_{1} x_{2} \\
\cdot\left(\left(x_{1} x_{2} x_{3}\right)^{\lambda+1} x_{1} x_{2}\right)^{\tau}\left(x_{1} x_{2} x_{3}\right)^{\sigma} x_{1} .
\end{gathered}
$$

The equation $E$ with $\lambda+1>\sigma$ can be reduced by the transformation

$$
\begin{aligned}
x_{4} \rightarrow x_{2} & \left(x_{3} x_{1} x_{2}\right)^{\lambda-\sigma}\left(\left(x_{1} x_{2} x_{3}\right)^{\lambda+1} x_{1} x_{2}\right)^{\kappa-\tau} x_{3} x_{1} x_{2} \\
\cdot & \cdot\left(\left(x_{1} x_{2} x_{3}\right)^{\lambda+1} x_{1} x_{2}\right)^{\tau}\left(x_{1} x_{2} x_{3}\right)^{\sigma} x_{1}
\end{aligned}
$$

to the equation $x_{1} x_{2} x_{3}=x_{2} x_{3} x_{1}$, that is, to the equation 2 .

The equation $E$ with $\lambda+1=\sigma$ and $\tau<\kappa$ can be reduced by the transformation

$$
\begin{gathered}
\left\{\begin{array}{l}
x_{1} \rightarrow x_{1}^{\eta}, \\
x_{2} \rightarrow x_{1}^{\theta},
\end{array}\right. \\
x_{4} \rightarrow x_{4} x_{1}^{\eta+\theta}\left(x_{3} x_{1}^{\eta+\theta}\right)^{\lambda}\left(\left(x_{1}^{\eta+\theta} x_{3}\right)^{\lambda+1} x_{1}^{\eta+\theta}\right)^{\kappa-\tau-1} \\
\left.\cdot x_{3} x_{1}^{\eta+\theta}\left(x_{1}^{\eta+\theta} x_{3}\right)^{\lambda+1} x_{1}^{\eta+\theta}\right)^{\tau}\left(x_{1}^{\eta+\theta} x_{3}\right)^{\lambda+1} x_{1}^{\eta}
\end{gathered}
$$

to the equation $x_{3} x_{4}=x_{1}^{\theta} x_{3}$, that is, to the equation $\mathbf{5}$. 
The equation $E$ with $\lambda+1=\sigma$ and $\tau=\kappa$ can be reduced by the transformation

$$
\begin{aligned}
& x_{2} \rightarrow x_{1}^{\beta} x_{2}, \\
& x_{1} \rightarrow x_{2} x_{1}, \\
&\left.\left.x_{4} \rightarrow\left(x_{2} x_{1}\right)^{\beta} x_{2}\left(\left(\left(x_{2} x_{1}\right)^{\beta+1} x_{2} x_{3}\right)^{\lambda+1}\left(x_{2} x_{1}\right)^{\beta+1} x_{2}\right)^{\tau}\right)\left(x_{2} x_{1}\right)^{\beta+1} x_{2} x_{3}\right)^{\sigma} x_{2} x_{1},
\end{aligned}
$$

then both $x_{1} \rightarrow x_{3} x_{1}$ and $x_{3} \rightarrow x_{1} x_{3}$, to the equation 2 .

The equation (jjjj) can be reduced by the transformation

$$
\begin{aligned}
& x_{1} \rightarrow x_{3} x_{2}\left(\left(x_{2} x_{3}\right)^{\lambda+1} x_{2}\right)^{\tau}\left(x_{2} x_{3}\right)^{\sigma} x_{2} x_{1}, \\
& x_{3} \rightarrow x_{1} x_{3}
\end{aligned}
$$

to the equation $F$ :

$$
\begin{aligned}
& x_{2} x_{1} x_{3} x_{4}= x_{3} x_{2}\left(x_{1} x_{3} x_{2}\right)^{\lambda-\sigma}\left(\left(x_{2} x_{1} x_{3}\right)^{\lambda+1} x_{2}\right)^{\kappa-\tau} x_{1} x_{3} x_{2} \\
& \cdot\left(\left(x_{2} x_{1} x_{3}\right)^{\lambda+1} x_{2}\right)^{\tau}\left(x_{2} x_{1} x_{3}\right)^{\sigma} x_{2} x_{1} \quad \text { with } \partial\left(x_{3}\right)>0 .
\end{aligned}
$$

The equation $F$ with $\lambda>\sigma$ can be reduced by the transformation

$$
\begin{gathered}
x_{4}=x_{3} x_{2}\left(x_{1} x_{3} x_{2}\right)^{\lambda-\sigma-1}\left(\left(x_{2} x_{1} x_{3}\right)^{\lambda+1} x_{2}\right)^{\kappa-\tau} x_{1} x_{3} x_{2} \\
\cdot\left(\left(x_{2} x_{1} x_{3}\right)^{\lambda+1} x_{2}\right)^{\tau}\left(x_{2} x_{1} x_{3}\right)^{\sigma} x_{2} x_{1}
\end{gathered}
$$

to the equation $x_{1} x_{2} x_{3}=x_{3} x_{1} x_{2}$, that is, to the equation $\mathbf{2}$ with $\partial\left(x_{3}\right)>0$. On the other hand, t7 is the parametric solution of $\mathbf{1 3}$.

The equation $F$ with $\lambda=\sigma$ and $\kappa>\tau$ can be reduced by the transformation

$$
\begin{gathered}
x_{4} \rightarrow x_{4} x_{3}\left(x_{2} x_{1} x_{3}\right)^{\lambda} x_{2}\left(\left(x_{2} x_{1} x_{3}\right)^{\lambda+1} x_{2}\right)^{\kappa-\tau-1} x_{1} x_{3} x_{2} \\
\cdot\left(\left(x_{2} x_{1} x_{3}\right)^{\lambda+1} x_{2}\right)^{\tau}\left(x_{2} x_{1} x_{3}\right)^{\lambda} x_{2} x_{1}
\end{gathered}
$$

to the equation $x_{2} x_{1} x_{3} x_{4}=x_{3} x_{2}^{2} x_{1}$ with $\partial\left(x_{3}\right)>0$, that is, to the equation 12 .

The equation $F$ with $\lambda=\sigma$ and $\kappa=\tau$ can be reduced by the transformation

$$
x_{4} \rightarrow x_{3} x_{2}\left(\left(x_{2} x_{1} x_{3}\right)^{\lambda+1} x_{2}\right)^{\kappa}\left(x_{2} x_{1} x_{3}\right)^{\lambda} x_{2} x_{1}
$$

to the equation $x_{2} x_{1} x_{3}=x_{3} x_{2} x_{1}$ with $\partial\left(x_{3}\right)>0$, that is, to the equation 2 with $\partial\left(x_{3}\right)>0$. On the other hand, t9 is the parametric solution of $\mathbf{1 3}$.

Proposition 14. The general solution of the equation

14

$$
x_{1} x_{2} x_{3} x_{4}=x_{3} x_{2}\left(x_{2}\left(x_{3} x_{2}\right)^{\lambda+1}\right)^{\tau+1} x_{1}
$$

where $\lambda$ and $\tau$ are natural parameters, is described by the transformations

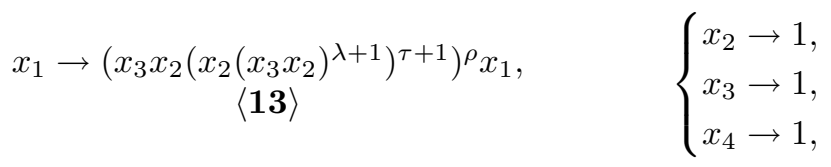

where $\rho$ is a natural parameter.

Proof. This follows directly from Propositions 8 and 13.

Proposition 15. The general solution of the equation

$$
x_{1} x_{2} x_{3} x_{4}=\left(x_{3} x_{1}\right)^{\tau+2} x_{2},
$$


where $\tau$ is a natural parameter, is described by the transformations

$$
\begin{aligned}
& x_{3} \rightarrow 1, \\
& \langle\mathbf{5}\rangle,
\end{aligned} \quad\left\{\begin{array}{l}
x_{1} \rightarrow\left(x_{1} x_{3}\right)^{\alpha} x_{1}, \\
x_{3} \rightarrow x_{1} x_{3},
\end{array}\right.
$$

where $\alpha$ is a natural parameter.

Proof. The equation $\mathbf{1 5}$ can be divided into the collection of the equations $\mathbf{1 5}$ with $\partial\left(x_{3}\right)=0$ and 15 with $\partial\left(x_{3}\right)>0$. In the second case we use Proposition 8.

Proposition 16. The general solution of the equation

16

$$
x_{1} x_{2} x_{3} x_{4}=x_{3} x_{2}^{\tau+2} x_{1}
$$

with $\partial\left(x_{1}\right)<\partial\left(x_{3} x_{2}^{\tau+2}\right)$ and $\partial\left(x_{3}\right)<\partial\left(x_{1} x_{2}\right)$, where $\tau$ is a natural parameter, is described by the transformations

$$
\begin{array}{cl}
\left\{\begin{array}{l}
x_{1} \rightarrow x_{3}\left(x_{1} x_{2}\right)^{\nu} x_{1}, \\
x_{2} \rightarrow x_{1} x_{2}, \\
x_{4} \rightarrow x_{4}\left(x_{1} x_{2}\right)^{\nu} x_{1},
\end{array}\right. & \left\{\begin{array}{l}
x_{1} \rightarrow x_{1}^{\eta}, \\
x_{2} \rightarrow x_{1}^{\theta},
\end{array}\right.
\end{array}
$$

$$
\left\{\begin{array}{l}
x_{3} \rightarrow x_{1} x_{3} \\
x_{2} \rightarrow x_{3} x_{2}
\end{array}\right.
$$

$\langle\mathbf{1 5}\rangle$,

where $\nu, \eta, \theta$ are natural parameters with $\nu \leq \tau$.

Proof. The equation $\mathbf{1 6}$ can be divided into the collection of equations

(j) 16 with $\partial\left(x_{3}\right) \leq \partial\left(x_{1}\right)$,

(jj) 16 with $\partial\left(x_{1}\right) \leq \partial\left(x_{3}\right)$.

The equation $(\mathrm{j})$ can be reduced by the transformation $x_{1} \rightarrow x_{3} x_{1}$ to the equation $E_{1}$ :

$$
x_{1} x_{2} x_{3} x_{4}=x_{2}^{\tau+2} x_{3} x_{1} \quad \text { with } \partial\left(x_{1}\right)<\partial\left(x_{2}^{\tau+2}\right) .
$$

The equation $E_{1}$ can be reduced by the transformation

$$
\begin{aligned}
& x_{1} \rightarrow x_{2}^{\nu} x_{1} \quad(\nu<\tau+2), \\
& x_{2} \rightarrow x_{1} x_{2}
\end{aligned}
$$

to the equation $E_{2}$ :

$$
x_{1} x_{2} x_{3} x_{4}=x_{2}\left(x_{1} x_{2}\right)^{\tau+1-\nu} x_{3}\left(x_{1} x_{2}\right)^{\tau} x_{1} .
$$

If $\tau+1>\nu$, then the equation $E_{2}$ falls into the system $E_{3}$ :

$$
\left\{\begin{array}{l}
x_{1} x_{2}=x_{2} x_{1}, \\
x_{3} x_{4}=x_{2}\left(x_{1} x_{2}\right)^{\tau-\nu} x_{3}\left(x_{1} x_{2}\right)^{\nu} x_{1} .
\end{array}\right.
$$

The system $E_{3}$ can be reduced by the transformation

$$
x_{4} \rightarrow x_{4}\left(x_{1} x_{2}\right)^{\tau} x_{1}
$$


to the system $E_{4}$ :

$$
\left\{\begin{array}{l}
x_{1} x_{2}=x_{2} x_{1}, \\
x_{3} x_{4}=x_{2}\left(x_{1} x_{2}\right)^{\tau-\nu} x_{3} .
\end{array}\right.
$$

By Proposition 1 the system $E_{4}$ is reduced by the parametric transformation

$$
\left\{\begin{array}{l}
x_{1} \rightarrow x_{1}^{\eta} \\
x_{2} \rightarrow x_{1}^{\theta}
\end{array}\right.
$$

to the equation

$$
x_{3} x_{4}=x_{1}^{\theta+(\nu+\theta)(\tau-\nu)} x_{3}
$$

that is, to the equation 5 .

If $\tau+1=\nu$, then the equation $E_{2}$ can be reduced by the transformation $x_{4} \rightarrow$ $\left(x_{2} x_{1}\right)^{\nu}$ to the equation $x_{1} x_{2} x_{3}=x_{2} x_{3} x_{1}$, that is, to the equation 2 .

The equation (jj) can be reduced by the transformation $x_{3} \rightarrow x_{1} x_{3}$ to the equation $E_{5}$ :

$$
x_{2} x_{1} x_{3} x_{4}=x_{3} x_{2}^{\tau+2} x_{1} \text { with } \partial\left(x_{3}\right)<\partial\left(x_{2}\right) .
$$

The equation $E_{5}$ can be reduced by the transformation $x_{2} \rightarrow x_{3} x_{2}$ to the equation

$$
x_{2} x_{1} x_{3} x_{4}=\left(x_{3} x_{2}\right)^{\tau+2} x_{1},
$$

that is the equation $\mathbf{1 5 .}$

Proposition 17. The general solution of the equation

17

$$
x_{1} x_{2} x_{3} x_{4}=x_{3} x_{2}^{\tau+2} x_{1},
$$

where $\tau$ is a natural parameter, is described by the transformation

$$
\left\{\begin{array}{l}
x_{1} \rightarrow{ }^{\mathbf{F i}}\left(x_{1} x_{2}, x_{3} x_{2}^{\tau+2}\right)^{\mu} x_{1}, \\
x_{3} \rightarrow \mathbf{F i}\left(x_{3} x_{2}^{\tau+2}, x_{1} x_{2}\right)^{\mu \mid} x_{3},
\end{array}\right.
$$

where $\mu$ is a variable whose values are sequences of natural parameters, followed by one of the three transformations

$$
\left\{\begin{array} { l } 
{ x _ { 2 } \rightarrow 1 , } \\
{ x _ { 3 } \rightarrow 1 , } \\
{ x _ { 4 } \rightarrow 1 , }
\end{array} \quad \left\{\begin{array}{l}
x_{1} \rightarrow 1 \\
x_{2} \rightarrow 1 \\
x_{4} \rightarrow 1
\end{array}\right.\right.
$$

Proof. This follows directly from Propositions 9 and 16.

Proposition 18. The general solution of the equation

18

$$
x_{1} x_{2} x_{3} x_{4}=x_{2}^{\sigma+3} x_{3} x_{1},
$$

where $\sigma$ is a natural parameter, is described by the transformations

$$
\begin{gathered}
x_{1} \rightarrow x_{2}^{\sigma+3} x_{1}, \\
\langle\mathbf{1 7}\rangle,
\end{gathered} \quad\left\{\begin{array}{l}
x_{1} \rightarrow\left(x_{1} x_{2}\right)^{\sigma+2} x_{1}, \\
x_{2} \rightarrow x_{1} x_{2}, \\
x_{4} \rightarrow\left(x_{2} x_{1}\right)^{\sigma+2}, \\
\langle\mathbf{2}\rangle,
\end{array} \quad\left\{\begin{array}{l}
x_{1} \rightarrow\left(x_{1} x_{2}\right)^{\nu} x_{1}, \\
x_{2} \rightarrow x_{1} x_{2}, \\
x_{4} \rightarrow x_{4}\left(x_{1} x_{2}\right)^{\nu} x_{1},
\end{array}\right\}\right.
$$


where $\nu, \eta, \theta$ are natural parameters with $\nu \leq \sigma+1$.

Proof. The equation $\mathbf{1 8}$ can be divided into the collection of equations

(j) 18 with $\partial\left(x_{2}^{\sigma+3}\right) \leq \partial\left(x_{1}\right)$,

(jj) 18 with $\partial\left(x_{2}^{\sigma+2}\right) \leq \partial\left(x_{1}\right)<\partial\left(x_{2}^{\sigma+3}\right)$,

(jjj) 18 with $\partial\left(x_{2}^{\nu}\right) \leq \partial\left(x_{1}\right)<\partial\left(x_{2}^{\nu+1}\right), \nu<\sigma+2$.

The equation $(\mathrm{j})$ can be reduced by the transformation $x_{1} \rightarrow x_{2}^{\sigma+3} x_{1}$ to the equation $x_{1} x_{2} x_{3} x_{4}=x_{3} x_{2}^{\sigma+3} x_{1}$, that is, to $\mathbf{1 7}$ with $\tau>0$.

The equation (jj) can be reduced by the transformation $x_{1} \rightarrow x_{2}^{\sigma+2} x_{1}, x_{2} \rightarrow$ $x_{1} x_{2}, x_{4} \rightarrow\left(x_{2} x_{1}\right)^{\sigma+2}$ to the equation $x_{1} x_{2} x_{3}=x_{2} x_{3} x_{1}$, that is, to $\mathbf{2}$.

The equation (jjj) can be reduced by the transformation $x_{1} \rightarrow x_{2}^{\nu} x_{1}$ with $\nu<$ $\sigma+2, x_{2} \rightarrow x_{1} x_{2}, x_{4} \rightarrow x_{4}\left(x_{1} x_{2}\right)^{\nu} x_{1}$ to the equation $E:$

$$
x_{1} x_{2} x_{3} x_{4}=x_{2} x_{1} x_{2}\left(x_{1} x_{2}\right)^{\sigma+1-\nu} x_{3} .
$$

By Proposition 1 the equation $E$ is reduced by the parametric transformation

$$
\left\{\begin{array}{l}
x_{1} \rightarrow x_{1}^{\eta}, \\
x_{2} \rightarrow x_{1}^{\theta}
\end{array}\right.
$$

to the equation

$$
x_{3} x_{4}=x_{1}^{\theta+(\eta+\theta)(\sigma+1-\nu)} x_{3},
$$

that is, to 5 .

Proposition 19. The general solution of the equation

19

$$
x_{1} x_{2} x_{3} x_{4}=x_{3} x_{1}^{\sigma+3} x_{2} \quad \text { with } \partial\left(x_{3}\right)<\partial\left(x_{1} x_{2}\right),
$$

where $\sigma$ is a natural parameter, is described by the transformations

$$
\begin{gathered}
x_{1} \rightarrow x_{3} x_{1}, \\
\langle\mathbf{1 5}\rangle
\end{gathered}, \quad\left\{\begin{array}{l}
x_{3} \rightarrow x_{1} x_{3}, \\
x_{2} \rightarrow x_{3} x_{2},
\end{array}\right.
$$

$\langle\mathbf{1 8}\rangle$.

Proof. The equation 19 can be divided into the collection of equations

(j) 19 with $\partial\left(x_{3}\right) \leq \partial\left(x_{1}\right)$,

(jj) 19 with $\partial\left(x_{3}\right) \geq \partial\left(x_{1}\right)$.

The equation ( $\mathrm{j}$ ) can be reduced by the transformation $x_{1} \rightarrow x_{3} x_{1}$ to the equation $x_{1} x_{2} x_{3} x_{4}=\left(x_{3} x_{1}\right)^{\sigma+3} x_{2}$, that is, to 15 with $\tau>0$.

The equation (jj) can be reduced by the transformation $x_{3} \rightarrow x_{1} x_{3}, x_{2} \rightarrow x_{3} x_{2}$ to the equation $x_{2} x_{1} x_{3} x_{4}=x_{1}^{\sigma+3} x_{3} x_{2}$, that is, to $\mathbf{1 8}$.

Proposition 20. The general solution of the equation

20

$$
x_{1} x_{2} x_{3} x_{4}=x_{3} x_{1}^{\tau+3} x_{2},
$$

where $\tau$ is a natural parameter, is described by the transformations

$$
\left\{\begin{array}{l}
x_{1} \rightarrow 1, \\
x_{2} \rightarrow 1, \\
x_{4} \rightarrow 1,
\end{array} \quad x_{3} \rightarrow\left(x_{1} x_{2}\right)^{\alpha} x_{3},\right.
$$

where $\alpha$ is a natural parameter.

Proof. The equation $\mathbf{2 0}$ can be divided into the collection of equations $\mathbf{2 0}$ with $\partial\left(x_{1} x_{2}\right)=0$ and 20 with $\partial\left(x_{1} x_{2}\right)>0$. In the second case we use Proposition 8 . 


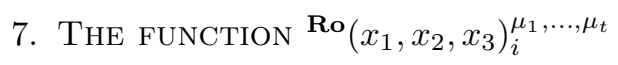

Consider the equation

$$
x_{1} x_{2} x_{3} x_{4}=x_{2} x_{3}^{2} x_{1}
$$

and a sequence of transformations (15):

$(15.2 k+1)$

$$
\begin{aligned}
& \left\{\begin{array}{l}
x_{1} \rightarrow \mathbf{F i}\left(x_{3} x_{2} x_{1}, x_{3} x_{2}\left(x_{1} x_{3}\right)^{2}\right)^{\mu_{1}} x_{3} x_{2} x_{1}, \\
x_{2} \rightarrow \mathbf{F i}\left(x_{3} x_{2}\left(x_{1} x_{3}\right)^{2}, x 3 x_{2} x_{1}\right)^{\mu_{1} \mid} x_{3} x_{2}, \\
x_{3} \rightarrow x_{1} x_{3} \\
x_{4} \rightarrow x_{4} x_{1},
\end{array}\right. \\
& \left\{\begin{array}{l}
x_{1} \rightarrow \mathbf{F i}\left(x_{3} x_{1}\left(x_{2} x_{3}\right)^{2}, x_{3} x_{1} x_{2}\right)^{\mu_{2} \mid} x_{3} x_{1}, \\
x_{2} \rightarrow \mathbf{F i}\left(x_{3} x_{1} x_{2}, x_{3} x_{1}\left(x_{2} x_{3}\right)^{2}\right)^{\mu_{2}} x_{3} x_{1} x_{2}, \\
x_{3} \rightarrow x_{2} x_{3} \\
x_{4} \rightarrow x_{4} x_{2}
\end{array}\right.
\end{aligned}
$$

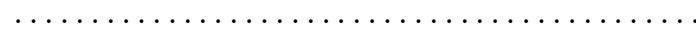$$
\left\{\begin{array}{l}
x_{1} \rightarrow{ }^{\mathbf{F i}}\left(x_{3} x_{2} x_{1}, x_{3} x_{2}\left(x_{1} x_{3}\right)^{2}\right)^{\mu_{2 k+1}} x_{3} x_{2} x_{1} \\
x_{2} \rightarrow \mathbf{F i}\left(x_{3} x_{2}\left(x_{1} x_{3}\right)^{2}, x_{3} x_{2} x_{1}\right)^{\mu_{2 k+1} \mid} x_{3} x_{2} \\
x_{3} \rightarrow x_{1} x_{3} \\
x_{4} \rightarrow x_{4} x_{1}
\end{array}\right.
$$$$
\left\{\begin{array}{l}
x_{1} \rightarrow \mathbf{F i}\left(x_{3} x_{1}\left(x_{2} x_{3}\right)^{2}, x_{3} x_{1} x_{2}\right)^{\mu_{2 k+2} \mid} x_{3} x_{1}, \\
x_{2} \rightarrow \mathbf{F i}\left(x_{3} x_{1} x_{2}, x_{3} x_{1}\left(x_{2} x_{3}\right)^{2}\right)^{\mu_{2 k+2}} x_{3} x_{1} x_{2}, \\
x_{3} \rightarrow x_{2} x_{3} \\
x_{4} \rightarrow x_{4} x_{2}
\end{array}\right.
$$

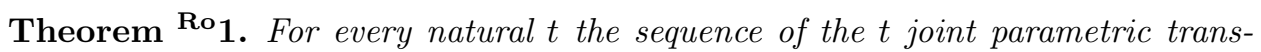
formations (15) can be collected by the following common transformation:

$$
\left\{\begin{array}{l}
x_{1} \rightarrow{ }^{\mathbf{R o}}\left(x_{1}, x_{2}, x_{3}\right)_{1}^{\mu_{1}, \ldots, \mu_{t}} x_{1}, \\
x_{2} \rightarrow \mathbf{R o}\left(x_{1}, x_{2}, x_{3}\right)_{2}^{\mu_{1}, \ldots, \mu_{t}} x_{2}, \\
x_{3} \rightarrow{ }^{\mathbf{R o}}\left(x_{1}, x_{2}, x_{3}\right)_{3}^{\mu_{1}, \ldots, \mu_{t}} x_{3}, \\
x_{4} \rightarrow x_{4}{ }^{\mathbf{R e}}\left(x_{1}, x_{2}, x_{3}\right)^{\mu_{1}, \ldots, \mu_{t}} .
\end{array}\right.
$$

Proof. If $t=0$, it is obvious. Let $t=1$. By the definition of $\mathbf{R o}\left(x_{1}, x_{2}, x_{3}\right)_{i}^{\mu_{1}, \ldots, \mu_{t}}$ we have

$$
\begin{gathered}
\mathbf{R o}_{\left(x_{1}, x_{2}, x_{3}\right){ }_{1}^{\mu_{1}}=}={ }^{\mathbf{F i}}\left(x_{3} x_{2} x_{1}, x_{3} x_{2}\left(x_{1} x_{3}\right)^{2}\right)^{\lambda_{1}, \ldots, \lambda_{s}} x_{3} x_{2}, \\
{ }^{\mathbf{R o}}\left(x_{1}, x_{2}, x_{3}\right)_{2}^{\mu_{1}}={ }^{\mathbf{F i}}\left(x_{3} x_{2}\left(x_{1} x_{3}\right)^{2}, x_{3} x_{2} x_{1}\right)^{\lambda_{2}, \ldots, \lambda_{s}} x_{3}, \\
{ }^{\mathbf{R o}}\left(x_{1}, x_{2}, x_{3}\right)_{3}^{\mu_{1}}=x_{1}, \\
\operatorname{Re}_{\left(x_{1}, x_{2}, x_{3}\right)^{\mu_{1}}=x_{1},}
\end{gathered}
$$

and the transformation (16) coincides with (15.1). 
Let $t>1$. By the inductive proposition the sequence of transformations (15.2), $\ldots,(15 . t)$ can be collected into the transformation

$$
\left\{\begin{array}{l}
x_{1} \rightarrow \mathbf{R o}\left(x_{2}, x_{1}, x_{3}\right)_{2}^{\mu_{2}, \ldots, \mu_{t}} x_{1}, \\
x_{2} \rightarrow \mathbf{R o}\left(x_{2}, x_{1}, x_{3}\right)_{1}^{\mu_{2}, \ldots, \mu_{t}} x_{2}, \\
x_{3} \rightarrow \mathbf{R o}\left(x_{2}, x_{1}, x_{3}\right)_{3}^{\mu_{2}, \ldots, \mu_{t}} x_{3}, \\
x_{4} \rightarrow x_{4}{ }^{\mathbf{R e}}\left(x_{2}, x_{1}, x_{3}\right)^{\mu_{2}, \ldots, \mu_{t}} .
\end{array}\right.
$$

Substituting (17) into (15.1), we obtain (16).

Proposition 21. The general solution of the equation

21

$$
x_{1} x_{2} x_{3} x_{4}=x_{2} x_{3}^{2} x_{1}
$$

is described by the transformations

$$
\left\{\begin{array}{l}
x_{1} \rightarrow \mathbf{F i}\left(x_{1}, x_{2} x_{3}^{2}\right)^{\mu} x_{1}, \\
x_{2} \rightarrow \mathbf{F i}\left(x_{2} x_{3}^{2}, x_{1}\right)^{\mu \mid} x_{2},
\end{array}\right.
$$

where $\mu$ is a variable for sequences of natural parameters, followed by one of the three transformations

$$
\left\{\begin{array} { l } 
{ x _ { 2 } \rightarrow 1 , } \\
{ x _ { 3 } \rightarrow 1 , } \\
{ x _ { 4 } \rightarrow 1 , }
\end{array} \quad \left\{\begin{array}{l}
x_{1} \rightarrow 1, \\
x_{4} \rightarrow x_{3},
\end{array}\right.\right.
$$

Proof. This follows directly from Propositions 9 and 22.

Proposition 22. The general solution of the equation

22

$$
x_{1} x_{2} x_{3} x_{4}=x_{2} x_{3}^{2} x_{1} \quad \text { with } \partial\left(x_{2}\right)<\partial\left(x_{1}\right)<\partial\left(x_{2} x_{3}^{2}\right)
$$

is described by the transformations $\langle\mathbf{2 3}\rangle,\langle\mathbf{2 4}\rangle,\langle\mathbf{2 5}\rangle$.

Proof. The equation $\mathbf{2 2}$ can be divided into the collection of equations:

(j) 22 with $\partial\left(x_{1}\right) \leq \partial\left(x_{3}\right)$,

(jj) 22 with $\partial\left(x_{2} x_{3}\right) \leq \partial\left(x_{1}\right)$,

(jjj) 22 with $\partial\left(x_{3}\right) \leq \partial\left(x_{1}\right) \leq \partial\left(x_{2} x_{3}\right)$.

By definition (j) is $\mathbf{2 3}$, (jj) is $\mathbf{2 4}$, (jjj) is $\mathbf{2 5}$.

Proposition 23. The general solution of the equation

23

$$
x_{1} x_{2} x_{3} x_{4}=x_{2} x_{3}^{2} x_{1},
$$

with $\partial\left(x_{2}\right)<\partial\left(x_{1}\right)<\partial\left(x_{2} x_{3}^{2}\right)$ and $\partial\left(x_{1}\right) \leq \partial\left(x_{3}\right)$, is described by the transformation

$$
\mathrm{t}\left\{\begin{array}{l}
x_{1} \rightarrow x_{2} x_{1} \\
x_{3} \rightarrow x_{1} x_{2} x_{3} \\
x_{4} \rightarrow x_{3} x_{2} x_{1}
\end{array}\right.
$$

Proof. The equation $\mathbf{2 3}$ can be reduced by the transformation $x_{1} \rightarrow x_{2} x_{1}$ to the equation $E_{1}$ :

$$
x_{1} x_{2} x_{3} x_{4}=x_{3}^{2} x_{2} x_{1} \quad \text { with } 0<\partial\left(x_{1}\right)<\partial\left(x_{3}^{2}\right), \partial\left(x_{2} x_{1}\right) \leq \partial\left(x_{3}\right) .
$$


The equation $E_{1}$ can be reduced by the transformation $x_{3} \rightarrow x_{1} x_{2} x_{3}$ to the equation $E_{2}$ :

$$
x_{1} x_{2} x_{3} x_{4}=x_{3} x_{1} x_{2} x_{3} x_{2} x_{1} \quad \text { with } \partial\left(x_{1}\right)>0 .
$$

The equation $E_{2}$ can be reduced by the transformation $x_{4} \rightarrow x_{3} x_{2} x_{1}$ to the equation $x_{1} x_{2} x_{3}=x_{3} x_{1} x_{2}$ with $\partial\left(x_{1}\right)>0$, that is, to the equation 2 with $\partial\left(x_{1}\right)>0$.

On the other hand, the transformation $\mathrm{t}$ is a parametric solution of $\mathbf{2 3}$.

Proposition 24. The general solution of the equation

$$
x_{1} x_{2} x_{3} x_{4}=x_{2} x_{3}^{2} x_{1},
$$

with $\partial\left(x_{2}\right)<\partial\left(x_{1}\right)<\partial\left(x_{2} x_{3}^{2}\right)$ and $\partial\left(x_{2} x_{3}\right) \leq \partial\left(x_{1}\right)$, is described by the transformation

$$
\mathrm{t}\left\{\begin{array}{l}
x_{1} \rightarrow x_{2} x_{3} x_{1} \\
x_{3} \rightarrow x_{1} x_{3} \\
x_{4} \rightarrow x_{3} x_{1}
\end{array}\right.
$$

Proof. The equation $\mathbf{2 4}$ can be reduced by the transformation $x_{1} \rightarrow x_{2} x_{3} x_{1}$ to the equation $E_{1}$ :

$$
x_{1} x_{2} x_{3} x_{4}=x_{3} x_{2} x_{3} x_{1} \quad \text { with } 0<\partial\left(x_{1}\right)<\partial\left(x_{3}\right) .
$$

The equation $E_{1}$ can be reduced by the transformation $x_{3} \rightarrow x_{1} x_{3}$ to the equation $E_{2}$ :

$$
x_{2} x_{1} x_{3} x_{4}=x_{3} x_{2} x_{1} x_{3} x_{1}
$$

with $\partial\left(x_{1}\right)>0, \partial\left(x_{3}\right)>0$. The equation $E_{2}$ can be reduced by the transformation $x_{4} \rightarrow x_{3} x_{1}$ to the equation $x_{2} x_{1} x_{3}=x_{3} x_{2} x_{1}$ with $\partial\left(x_{1}\right)>0, \partial\left(x_{3}\right)>0$, that is, to the equation 2 with $\partial\left(x_{1}\right)>0, \partial\left(x_{3}\right)>0$.

On the other hand, the transformation $t$ is a parametric solution of $\mathbf{2 4}$.

Proposition 25. The general solution of the equation

$$
x_{1} x_{2} x_{3} x_{4}=x_{2} x_{3}^{2} x_{1},
$$

with $\partial\left(x_{2}\right)<\partial\left(x_{1}\right)<\partial\left(x_{2} x_{3}^{2}\right)$ and $\partial\left(x_{3}\right) \leq \partial\left(x_{1}\right) \leq \partial\left(x_{2} x_{3}\right)$, is described by the transformation

$$
\begin{array}{r}
\mathrm{t}\left\{\begin{array}{l}
x_{1} \rightarrow x_{2} x_{1}, \\
x_{3} \rightarrow x_{1} x_{3}, \\
x_{2} \rightarrow x_{3} x_{2}, \\
x_{4} \rightarrow x_{4} x_{1},
\end{array}\right. \\
\left\{\begin{array}{l}
x_{1} \rightarrow x_{2}, \\
x_{2} \rightarrow x_{1},
\end{array}\right.
\end{array}
$$

$\langle\mathbf{2 1}\rangle$.

Proof. The equation $\mathbf{2 5}$ can be reduced by the transformation $x_{1} \rightarrow x_{2} x_{1}$ to the equation $E_{1}$ :

$$
x_{1} x_{2} x_{3} x_{4}=x_{3}^{2} x_{2} x_{1}
$$


with $0<\partial\left(x_{1}\right)<\partial\left(x_{3}^{2}\right)$ and $\partial\left(x_{3}\right) \leq \partial\left(x_{1} x_{2}\right)$. The equation $E_{1}$ can be reduced by the transformation $x_{3} \rightarrow x_{1} x_{3}$ to the equation $E_{2}$ :

$$
x_{2} x_{1} x_{3} x_{4}=x_{3} x_{1} x_{3} x_{2} x_{1}
$$

with $\partial\left(x_{3}\right) \leq \partial\left(x_{2}\right)$ and $\partial\left(x_{1}\right)>0$. The equation $E_{2}$ can be reduced by the transformations $x_{2} \rightarrow x_{3} x_{2}, x_{4} \rightarrow x_{4} x_{1}$ to the equation $E_{3}$ :

$$
x_{2} x_{1} x_{3} x_{4}=x_{1} x_{3}^{2} x_{2}
$$

with $\partial\left(x_{1}\right)>0$. The equation $E_{3}$ can be reduced by the transformation

$$
\left\{\begin{array}{l}
x_{1} \rightarrow x_{2} \\
x_{2} \rightarrow x_{1}
\end{array}\right.
$$

to the equation $\mathbf{2 1}$ with $\partial\left(x_{2}\right)>0$.

On the other hand, the transformation t is a parametric solution of $\mathbf{2 5}$.

Proposition 26. The general solution of the equation

26

$$
x_{1} x_{2} x_{3} x_{4}=x_{2} x_{3}^{2} x_{1}
$$

is described by the transformation

$$
\mathrm{t}\left\{\begin{array}{l}
x_{1} \rightarrow \mathbf{F i}\left(x_{1}, x_{2} x_{3}^{2}\right)^{\mu} x_{1}, \\
x_{2} \rightarrow \mathbf{F i}\left(x_{2} x_{3}^{2}, x_{1}\right)^{\mu \mid} x_{2}
\end{array}\right.
$$

where $\mu$ is a variable for sequences of natural parameters, followed by one of the four transformations

$$
\left\{\begin{array} { l } 
{ x _ { 2 } \rightarrow 1 , } \\
{ x _ { 3 } \rightarrow 1 , } \\
{ x _ { 4 } \rightarrow 1 , }
\end{array} \quad \left\{\begin{array}{l}
x_{1} \rightarrow 1, \\
x_{4} \rightarrow x_{3},
\end{array} \quad\langle\mathbf{2 3}\rangle, \quad\langle\mathbf{2 4}\rangle\right.\right.
$$

and the transformation

$$
\text { tt }\left\{\begin{array}{l}
x_{1} \rightarrow \mathbf{F i}\left(x_{3} x_{2} x_{1}, x_{3} x_{2}\left(x_{1} x_{3}\right)^{2}\right)^{\mu} x_{3} x_{2} x_{1}, \\
x_{2} \rightarrow \mathbf{F i}\left(x_{3} x_{2}\left(x_{1} x_{3}\right)^{2}, x_{3} x_{2} x_{1}\right)^{\mu \mid} x_{3} x_{2}, \\
x_{3} \rightarrow x_{1} x_{3} \\
x_{4} \rightarrow x_{4} x_{1}
\end{array}\right.
$$

where $\mu$ is a variable for sequences of natural parameters, followed by the transformation

$$
\left\{\begin{array}{l}
x_{1} \rightarrow x_{2} \\
x_{2} \rightarrow x_{1}
\end{array}\right.
$$

$\langle\mathbf{2 1}\rangle$.

Proof. Proposition 26 is deduced by means of Propositions 21-25.

Proposition 27. The general solution of the equation

$$
x_{1} x_{2} x_{3} x_{4}=x_{2} x_{3}^{2} x_{1}
$$


is described by the transformation

$$
\mathrm{T}\left\{\begin{array}{l}
x_{1} \rightarrow \mathbf{R o}\left(x_{1}, x_{2}, x_{3}\right)_{1}^{\nu} x_{1} \\
x_{2} \rightarrow \mathbf{R o}\left(x_{1}, x_{2}, x_{3}\right)_{2}^{\nu} x_{2} \\
x_{3} \rightarrow \mathbf{R o}\left(x_{1}, x_{2}, x_{3}\right)_{3}^{\nu} x_{3} \\
x_{4} \rightarrow x_{4} \operatorname{Re}_{\left(x_{1}, x_{2}, x_{3}\right)^{\nu}}
\end{array}\right.
$$

where $\nu$ is a variable whose values are even sequences of variables from the alphabet (4), followed by the transformation

$$
\left\{\begin{array}{l}
x_{1} \rightarrow{ }^{\mathbf{F i}}\left(x_{1}, x_{2} x_{3}^{2}\right)^{\mu} x_{1}, \\
x_{2} \rightarrow \mathbf{F i}\left(x_{2} x_{3}^{2}, x_{1}\right)^{\mu \mid} x_{2},
\end{array}\right.
$$

where $\mu$ is a variable whose values are finite sequences of natural parameters, followed by one of the four transformations

$$
\left\{\begin{array} { l } 
{ x _ { 2 } \rightarrow 1 , } \\
{ x _ { 3 } \rightarrow 1 } \\
{ x _ { 4 } \rightarrow 1 }
\end{array} \quad \left\{\begin{array}{l}
x_{1} \rightarrow 1 \\
x_{4} \rightarrow x_{3}
\end{array}\right.\right.
$$

and the transformation

$$
\mathrm{TT}\left\{\begin{array}{l}
x_{1} \rightarrow \mathbf{R o}\left(x_{1}, x_{2}, x_{3}\right){ }_{1}^{\kappa} x_{1}, \\
x_{2} \rightarrow \operatorname{Ro}\left(x_{1}, x_{2}, x_{3}\right)_{2}^{\kappa} x_{2}, \\
x_{3} \rightarrow \operatorname{Ro}\left(x_{1}, x_{2}, x_{3}\right)_{3}^{\kappa} x_{3}, \\
x_{4} \rightarrow x_{4}{ }^{\mathbf{R e}}\left(x_{1}, x_{2}, x_{3}\right)^{\kappa},
\end{array}\right.
$$

where $\kappa$ is a variable whose values are odd sequences of variables from the alphabet (4), followed by the transformation

$$
\left\{\begin{array}{l}
x_{1} \rightarrow{ }^{\mathbf{F i}}\left(x_{1} x_{3}^{2}, x_{2}\right)^{\mu \mid} x_{1}, \\
x_{2} \rightarrow \mathbf{F i}\left(x_{2}, x_{1} x_{3}^{2}\right)^{\mu} x_{2},
\end{array}\right.
$$

where $\mu$ is a variable whose values are finite sequences of natural parameters, followed by one of the four transformations

$$
\left\{\begin{array} { l } 
{ x _ { 1 } \rightarrow 1 , } \\
{ x _ { 3 } \rightarrow 1 , } \\
{ x _ { 4 } \rightarrow 1 , }
\end{array} \quad \left\{\begin{array} { l } 
{ x _ { 2 } \rightarrow 1 , } \\
{ x _ { 4 } \rightarrow x _ { 3 } , }
\end{array} \quad \left\{\begin{array} { c } 
{ x _ { 1 } \rightarrow x _ { 2 } , } \\
{ x _ { 2 } \rightarrow x _ { 1 } , } \\
{ \langle \mathbf { 2 3 } \rangle , }
\end{array} \quad \left\{\begin{array}{c}
x_{1} \rightarrow x_{2}, \\
x_{2} \rightarrow x_{1}, \\
\langle\mathbf{2 4}\rangle .
\end{array}\right.\right.\right.\right.
$$

Proof. It is easy to apply the transformations $\mathrm{T}$ and TT to the equation $\mathbf{2 7}$ and

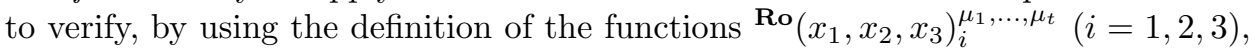
that they are parametric solution $\mathbf{2 7}$.

Let

$$
\text { S }\left\{\begin{array}{l}
x_{1} \rightarrow X_{1} \\
x_{2} \rightarrow X_{2} \\
x_{3} \rightarrow X_{3} \\
x_{4} \rightarrow X_{4}
\end{array}\right.
$$

where the $X_{i}$ are words in the alphabet (1), be an arbitrary solution of $\mathbf{2 7}$. We prove by induction on $\left|X_{1} X_{2} X_{3} X_{4}\right|$ that $\mathrm{S}$ is contained either in the transformation $\mathrm{T}$ or in $\mathrm{TT}$.

The equation $\mathbf{2 7}$ can be divided into the following collection of equations: 
(j) $\mathbf{2 7}$ with $\partial\left(x_{2}\right) \leq \partial\left(x_{1}\right) \leq \partial\left(x_{2} x_{3}^{2}\right)$,

(jj) 27 with $\partial\left(x_{1}\right) \leq \partial\left(x_{2}\right)$,

(jjj) 27 with $\partial\left(x_{2} x_{3}^{2}\right) \leq \partial\left(x_{1}\right)$.

Let $\mathrm{S}$ be a solution of $(\mathrm{j})$. By Proposition $22, \mathrm{~S}$ is contained in $\langle\mathbf{2 3}\rangle,\langle\mathbf{2 4}\rangle,\langle\mathbf{2 5}\rangle$.

If $\mathrm{S}$ is contained in $\langle\mathbf{2 3}\rangle$ or $\langle\mathbf{2 4}\rangle$, then $\mathrm{S}$ is contained in $\mathrm{T}$ with $\nu=\varnothing$ and $\mu=\varnothing$.

Let $\mathrm{S}$ be contained in $\langle\mathbf{2 5}\rangle$. If $\left|X_{1} X_{2} X_{3}\right|=0$, then $\mathrm{S}$ is contained in $\mathrm{T}$. Let $\left|X_{1} X_{2} X_{3}\right|>0$. Then $X_{1}=X_{2} Y_{1}$ for some $Y_{1}$, and $Y_{1} X_{2} X_{3} X_{4}=X_{3}^{2} X_{2} Y_{1}$. Then $X_{3}=Y_{1} Y_{3}$ and $X_{2} Y_{1} Y_{3} X_{4}=Y_{3} Y_{1} Y_{3} X_{2} Y_{1}$. But then $X_{2}=Y_{3} Y_{2}$ and $Y_{2} Y_{1} Y_{3} X_{4}=$ $Y_{1} Y_{3} Y_{3} Y_{2} Y_{1}$. By Proposition 25 the equation 25 is reduced by the parametric transformation

$$
\begin{aligned}
& \mathrm{t}\left\{\begin{array}{l}
x_{1} \rightarrow x_{3} x_{2} x_{1}, \\
x_{2} \rightarrow x_{3} x_{2}, \\
x_{3} \rightarrow x_{1} x_{3}, \\
x_{4} \rightarrow x_{4} x_{1},
\end{array}\right. \\
& \mathrm{c}\left\{\begin{array}{l}
x_{1} \rightarrow x_{2}, \\
x_{2} \rightarrow x_{1}
\end{array}\right.
\end{aligned}
$$

to the equation $\mathbf{2 1}$, that is, to $\mathbf{2 7}$. The image of the solution $\mathrm{S}$ via the transformation $\mathrm{t}$ is the coefficient transformation

$$
\mathrm{S}_{1}\left\{\begin{array}{l}
x_{1} \rightarrow Y_{1}, \\
x_{2} \rightarrow Y_{2}, \\
x_{3} \rightarrow Y_{3}, \\
x_{4} \rightarrow X_{4} .
\end{array}\right.
$$

Since $\left|Y_{1} Y_{2} Y_{3} X_{4}\right|<\left|X_{1} X_{2} X_{3} X_{4}\right|$, one can use the inductive proposition to see that $\mathrm{S}_{1}$ is contained in the parametric solutions cT, cTT. According to Lemma 1 the parametric solutions tcT, tcTT of $\mathbf{2 7}$ contain the solution $\mathrm{tcS}_{1}$ of $\mathbf{2 7}$, that is, $\mathrm{S}$.

According to Theorem ${ }^{\text {Ro }} 1$ the transformations tcT and tcTT can be obtained from $\mathrm{T}$ and TT by replacing the parameter $\nu$ by $\varnothing, \nu$.

Let $\mathrm{S}$ be a solution of $\mathrm{t}$ equation (jj). If $\left|X_{1}\right|=0$, then $\mathrm{S}$ is contained in $\mathrm{T}$ and TT. Let $\left|X_{1}\right|>0$. Then $X_{2}=X_{1} Y_{2}$ for some $Y_{2}$, and $X_{1} Y_{2} X_{3} X_{4}=Y_{2} X_{3}^{2} X_{1}$. The equation $\mathbf{2 7}$ is reduced by the transformation $\mathrm{t}: x_{2} \rightarrow x_{1} x_{2}$ to $\mathbf{2 7}$. The image of the solution $\mathrm{S}$ via the transformation $\mathrm{t}$ is the coefficient transformation

$$
\mathrm{S}_{1}\left\{\begin{array}{l}
x_{1} \rightarrow X_{1}, \\
x_{2} \rightarrow Y_{2}, \\
x_{3} \rightarrow X_{3}, \\
x_{4} \rightarrow X_{4} .
\end{array}\right.
$$

Since $\left|Y_{2}\right|<\left|X_{2}\right|$, one can use the inductive proposition to see that $\mathrm{S}_{1}$ is contained in T and TT. According to Lemma 1 the parametric solution tT, tTT of $\mathbf{2 7}$ contains the solution $\mathrm{tS}_{1}$, that is, $\mathrm{S}$.

According to Theorems ${ }^{\mathbf{F i}} 1$ and ${ }^{\mathrm{Ro}} 1$ the transformations tT and tTT can be obtained from T and TT by replacing the parameters $\nu, \mu$ by $\nu^{\prime}, \mu^{\prime}$, where if $\nu=\varnothing$, then $\nu^{\prime}=\varnothing$ and $\mu^{\prime}=0,1, \mu$; if $\nu=\mu_{1}, \ldots, \mu_{s}(s \geq 1)$, then $\nu^{\prime}=\mu_{1}^{\prime}, \ldots, \mu_{s}$, where $\mu_{1}^{\prime}=0,1, \mu_{1}$ and $\mu^{\prime}=\mu$.

Let $\mathrm{S}$ be a solution of the equation (jjj). If $\left|X_{2} X_{3}^{2}\right|=0$, then $\mathrm{S}$ is contained in T, TT. Let $\left|X_{2} X_{3}^{2}\right|>0$. Then $X_{1}=X_{2} X_{3}^{2} Y_{1}$ for some $Y_{1}$, and $Y_{1} X_{2} X_{3} X_{4}=$ 
$X_{2} X_{3}^{2} Y_{1}$. The equation $\mathbf{2 7}$ is reduced by the transformation $\mathrm{t}: x_{1} \rightarrow x_{2} x_{3}^{2} x_{1}$ to the equation 27. The image of the solution $\mathrm{S}$ via the transformation $\mathrm{t}$ is the coefficient transformation

$$
\mathrm{S}_{1}\left\{\begin{array}{l}
x_{1} \rightarrow Y_{1}, \\
x_{2} \rightarrow X_{2}, \\
x_{3} \rightarrow X_{3}, \\
x_{4} \rightarrow X_{4} .
\end{array}\right.
$$

Since $\left|Y_{1}\right|<\left|X_{1}\right|$, one can use the inductive proposition to see that $\mathrm{S}_{1}$ is contained in T and TT. According to Lemma 1 a parametric solution tT, tTT of 27 contains the solution $\mathrm{tS}_{1}$, that is, $\mathrm{S}$.

According to Theorems ${ }^{\mathbf{F i}} 1$ and ${ }^{\mathbf{R o}} 1$ the transformations tT and tTT can be obtained from $\mathrm{T}$ and TT by replacing the parameters $\nu, \mu$ by parameters $\nu^{\prime}, \mu^{\prime}$, where if $\nu=\varnothing$, then $\nu^{\prime}=\varnothing$ and $\mu^{\prime}=1,0, \mu$; if $\nu=\mu_{1}, \ldots, \mu_{s}(s \geq 1)$, then $\nu^{\prime}=\mu_{1}^{\prime}, \ldots, \mu_{s}$, where $\mu_{1}^{\prime}=1,0, \mu_{1}$ and $\mu^{\prime}=\mu$.

\section{Equations and solutions}

Proposition 28. The general solution of the equation

28

$$
x_{1} x_{2} x_{3} x_{4}=x_{3}\left(x_{2} x_{3}\right)^{\alpha+1} x_{1} \quad \text { with } \partial\left(x_{1}\right)<\partial\left(x_{3}\left(x_{2} x_{3}\right)^{\alpha+1}\right),
$$

where $\lambda$ is a natural parameter, is described by the transformations

$$
\mathrm{t}\left\{\begin{array}{l}
x_{1} \rightarrow\left(x_{3} x_{2}\right)^{\tau} x_{1} \\
x_{3} \rightarrow x_{1} x_{3} \\
x_{4} \rightarrow\left(x_{3} x_{2} x_{1}\right)^{\alpha-\tau} x_{3} x_{1}\left(x_{3} x_{2} x_{1}\right)^{\tau}
\end{array}\right.
$$

$\mathrm{tt}\left\{\begin{array}{l}x_{1} \rightarrow\left(x_{3} x_{2}\right)^{\tau} x_{3} x_{1} \\ x_{2} \rightarrow x_{1} x_{2} \\ x_{4} \rightarrow x_{2} x_{3}\left(x_{1} x_{2} x_{3}\right)^{\alpha-\tau-1} x_{3} x_{1}\left(x_{2} x_{3} x_{1}\right)^{\tau}\end{array}\right.$

$\operatorname{ttt}\left\{\begin{array}{l}x_{1} \rightarrow x_{3}\left(x_{2} x_{3}\right)^{\alpha} x_{1}, \\ x_{2} \rightarrow x_{1} x_{2}, \\ x_{4} \rightarrow x_{4}\left(x_{2} x_{3} x_{1}\right)^{\alpha},\end{array}\right.$

$\langle\mathbf{2 7}\rangle$,

$\operatorname{tttt}\left\{\begin{array}{l}x_{1} \rightarrow\left(x_{3} x_{2}\right)^{\alpha+1} x_{1}, \\ x_{3} \rightarrow x_{1} x_{3}, \\ x_{2} \rightarrow x_{3} x_{2}, \\ x_{4} \rightarrow x_{4}\left(x_{1} x_{3}^{2} x_{2}\right)^{\alpha} x_{1},\end{array}\right.$

$\langle\mathbf{2 7}\rangle$,

$\operatorname{ttttt}\left\{\begin{array}{l}x_{1} \rightarrow\left(x_{3} x_{2}\right)^{\alpha+1} x_{1}, \\ x_{3} \rightarrow x_{1} x_{2} x_{3}, \\ x_{4} \rightarrow x_{3} x_{2} x_{1}\left(x_{2} x_{3} x_{2} x_{1}\right)^{\alpha},\end{array}\right.$

$\langle\mathbf{2}\rangle$. 
Proof. The equation $\mathbf{2 8}$ can be divided into the collection of equations:

(j) 28 with $\partial\left(\left(x_{3} x_{2}\right)^{\tau}\right) \leq \partial\left(x_{1}\right)<\partial\left(x_{3}\left(x_{2} x_{3}\right)^{\tau}\right), \tau \leq \alpha$,

(jj) 28 with $\left.\partial\left(x_{3}\left(x_{2} x_{3}\right)^{\tau}\right) \leq \partial\left(x_{1}\right)<\partial\left(x_{3} x_{2}\right)^{\tau+1}\right), \tau \leq \alpha$,

(jjj) 28 with $\partial\left(\left(x_{3} x_{2}\right)^{\alpha+1}\right) \leq \partial\left(x_{1}\right)<\partial\left(x_{3}\left(x_{2} x_{3}\right)^{\alpha+1}\right)$.

The equation $(\mathrm{j})$ can be reduced by the transformation

$$
\begin{aligned}
& x_{1} \rightarrow\left(x_{3} x_{2}\right)^{\tau} x_{1}, \\
& x_{3} \rightarrow x_{1} x_{3}, \\
& x_{4} \rightarrow\left(x_{3} x_{2} x_{1}\right)^{\alpha-\tau} x_{3} x_{1}\left(x_{3} x_{2} x_{1}\right)^{\tau}
\end{aligned}
$$

to the equation $x_{2} x_{1} x_{3}=x_{3} x_{2} x_{1}$ with $\partial\left(x_{3}\right)>0$, that is, to $\mathbf{2}$ with $\partial\left(x_{3}\right)>0$. On the other hand, the transformation $\mathrm{t}$ is a parametric solution of $\mathbf{2 8}$.

The equation (jj) can be reduced by the transformation

$$
\begin{aligned}
& x_{1} \rightarrow x_{3}\left(x_{2} x_{3}\right)^{\tau} x_{1}, \\
& x_{2} \rightarrow x_{1} x_{2}
\end{aligned}
$$

to the equation $E$ :

$$
x_{1} x_{2} x_{3} x_{4}=x_{2} x_{3}\left(x_{1} x_{2} x_{3}\right)^{\alpha-\tau} x_{3} x_{1}\left(x_{2} x_{3} x_{1}\right)^{\tau}
$$

with $\partial\left(x_{2}\right)>0$. If $\tau<\alpha$, then the equation $E$ can be reduced by the transformation

$$
x_{4} \rightarrow x_{2} x_{3}\left(x_{1} x_{2} x_{3}\right)^{\alpha-\tau-1} x_{3} x_{1}\left(x_{2} x_{3} x_{1}\right)^{\tau}
$$

to the equation $x_{1} x_{2} x_{3}=x_{2} x_{3} x_{1}$ with $\partial\left(x_{2}\right)>0$, that is, to 2 with $\partial\left(x_{2}\right)>0$. On the other hand, the transformation tt is a parametric solution of $\mathbf{2 8}$. If $\tau=\alpha$, then the equation $E$ can be reduced by the transformation

$$
x_{4} \rightarrow x_{4}\left(x_{2} x_{3} x_{1}\right)^{\tau}
$$

to the equation $x_{1} x_{2} x_{3} x_{4}=x_{2} x_{3}^{2} x_{1}$ with $\partial\left(x_{2}\right)>0$, that is, to $\mathbf{2 7}$ with $\partial\left(x_{2}\right)>0$. On the other hand, the transformation ttt is a parametric solution of $\mathbf{2 8}$.

The equation (jjj) can be reduced by the transformation

$$
\begin{aligned}
& x_{1} \rightarrow\left(x_{3} x_{2}\right)^{\alpha+1} x_{1}, \\
& x_{3} \rightarrow x_{1} x_{3}
\end{aligned}
$$

to the equation $E_{1}$ :

$$
x_{2} x_{1} x_{3} x_{4}=x_{3} x_{1}\left(x_{3} x_{2} x_{1}\right)^{\alpha+1} \quad \text { with } \partial\left(x_{3}\right)>0 .
$$

The equation $E_{1}$ can be divided by means of the conditions $\partial\left(x_{2}\right) \geq \partial\left(x_{3}\right)$ and $\partial\left(x_{3}\right) \geq \partial\left(x_{2}\right)$.

The equation $E_{1}$ with $\partial\left(x_{2}\right) \geq \partial\left(x_{3}\right)$ can be reduced by the transformation

$$
\begin{aligned}
& x_{2} \rightarrow x_{3} x_{2}, \\
& x_{4} \rightarrow x_{4}\left(x_{1} x_{3}^{2} x_{2}\right)^{\alpha} x_{1}
\end{aligned}
$$

to the equation $x_{2} x_{1} x_{3} x_{4}=x_{1} x_{3}^{2} x_{2}$ with $\partial\left(x_{3}\right)>0$, that is, to $\mathbf{2 7}$ with $x_{1} \leftrightarrow x_{2}$ and $\partial\left(x_{3}\right)>0$. On the other hand, the transformation tttt is a parametric solution of 28.

The equation $E_{1}$ with $\partial\left(x_{3}\right) \geq \partial\left(x_{2}\right)$ can be reduced by the transformation

$$
\begin{aligned}
& x_{3} \rightarrow x_{2} x_{3}, \\
& x_{4} \rightarrow x_{3} x_{2} x_{1}\left(x_{2} x_{3} x_{2} x_{1}\right)^{\alpha}
\end{aligned}
$$

to the equation $x_{1} x_{2} x_{3}=x_{3} x_{1} x_{2}$, that is, to $\mathbf{2}$. 
Proposition 29. The general solution of the equation

29

$$
x_{1} x_{2} x_{3} x_{4}=x_{3}\left(x_{2} x_{3}\right)^{\alpha+1} x_{1},
$$

where $\lambda$ is a natural parameter, is described by the transformations

$$
\mathrm{t}\left\{\begin{array}{lc}
x_{2} \rightarrow 1, \\
x_{3} \rightarrow 1, \\
x_{4} \rightarrow 1,
\end{array} \quad x_{1} \rightarrow\left(x_{3}\left(x_{2} x_{3}\right)^{\alpha+1}\right)^{\beta} x_{1},\right.
$$

where $\beta$ is a natural parameter.

Proof. The equation $\mathbf{2 9}$ can be divided into a collection of equations by means of the conditions $\partial\left(x_{2} x_{3}\right)=0$ and $\partial\left(x_{2} x_{3}\right)>0$.

The equation 29 with $\partial\left(x_{2} x_{3}\right)=0$ can be reduced by the transformation $\mathrm{t}$ to the equation 1.

The equation 29 with $\partial\left(x_{2} x_{3}\right)>0$ by Proposition 8 can be reduced by the transformation $x_{1} \rightarrow\left(x_{3}\left(x_{2} x_{3}\right)^{\alpha+1}\right)^{\beta} x_{1}$, where $\beta$ is a natural parameter, to 28 .

Proposition 30. The general solution of the equation

30

$$
x_{1} x_{2} x_{3} x_{4}=x_{2} x_{3}^{\alpha+3} x_{1} \quad \text { with } \partial\left(x_{2}\right)<\partial\left(x_{1}\right)<\partial\left(x_{2} x_{3}^{\alpha+3}\right),
$$

where $\alpha$ is a natural parameter, is described by the transformations

$$
\begin{array}{cc}
x_{1} \rightarrow x_{2} x_{3}^{\alpha+2} x_{1}, & x_{1} \rightarrow x_{2} x_{3}^{\beta} x_{1}, \\
x_{3} \rightarrow x_{1} x_{3}, & x_{3} \rightarrow x_{1} x_{3}, \\
x_{4} \rightarrow\left(x_{3} x_{1}\right)^{\alpha+2}, & x_{4} \rightarrow x_{4}\left(x_{1} x_{3}\right)^{\beta} x_{1}, \\
\langle\mathbf{2}\rangle, & \langle\mathbf{2 9}\rangle,
\end{array}
$$$$
\langle\mathbf{2 9}\rangle \text {, }
$$

where $\beta$ is a natural parameter with $\beta \leq \alpha+2$.

Proof. The equation $\mathbf{3 0}$ can be reduced by the transformation

$$
\begin{aligned}
& x_{1} \rightarrow x_{2} x_{3}^{\beta} x_{1} \quad(\beta \leq \alpha+2), \\
& x_{3} \rightarrow x_{1} x_{3}
\end{aligned}
$$

to the equation $E$ :

$$
x_{2} x_{1} x_{3} x_{4}=x_{3}\left(x_{1} x_{3}\right)^{\alpha+2-\beta} x_{2}\left(x_{1} x_{3}\right)^{\beta} x_{1} .
$$

The equation $E$ can be divided into a collection of equations by means of the conditions $\beta=\alpha+2$ and $\beta<\alpha+2$.

The equation $E$ with $\beta=\alpha+2$ can be reduced by the transformation $x_{4} \rightarrow$ $\left(x_{3} x_{1}\right)^{\alpha+2}$ to the equation $x_{2} x_{1} x_{3}=x_{3} x_{2} x_{1}$, that is, to $\mathbf{2}$.

The equation $E$ with $\beta<\alpha+2$ can be reduced by the transformation $x_{4} \rightarrow$ $x_{4}\left(x_{1} x_{3}\right)^{\beta} x_{1}$ to the equation

$$
x_{2} x_{1} x_{3} x_{4}=x_{3}\left(x_{1} x_{3}\right)^{\alpha+2-\beta} x_{2},
$$

that is, to $\mathbf{2 9}$.

Proposition 31. The general solution of the equation

31

$$
x_{1} x_{2} x_{3} x_{4}=x_{2} x_{3}^{\alpha+3} x_{1},
$$

where $\alpha$ is a natural parameter, is described by the transformation

$$
\left\{\begin{array}{l}
x_{1} \rightarrow{ }^{\mathbf{F i}}\left(x_{1}, x_{2} x_{3}^{\alpha+3}\right)^{\mu} x_{1}, \\
x_{2} \rightarrow \mathbf{F i}\left(x_{2} x_{3}^{\alpha+3}, x_{1}\right)^{\mu \mid} x_{2},
\end{array}\right.
$$


where $\mu$ is a variable for sequences of natural parameters, followed by one of the three transformations

$$
\left\{\begin{array} { l } 
{ x _ { 2 } \rightarrow 1 , } \\
{ x _ { 3 } \rightarrow 1 , } \\
{ x _ { 4 } \rightarrow 1 , }
\end{array} \quad \left\{\begin{array}{l}
x_{1} \rightarrow 1, \\
x_{4} \rightarrow x_{3}^{\alpha+2}
\end{array}\right.\right.
$$

Proof. This follows directly from Propositions 9 and 30.

Proposition 32. The general solution of the equation

32

$$
x_{1} x_{2} x_{3} x_{4}=x_{2} x_{3}^{\alpha} x_{1} x_{5},
$$

where $\lambda$ is a natural parameter, is described by the transformations

$$
\begin{gathered}
\mathrm{t}\left\{\begin{array}{l}
\alpha \rightarrow 0, \\
x_{5} \rightarrow x_{3} x_{4},
\end{array}\right. \\
\langle\mathbf{1}\rangle,
\end{gathered} \quad \text { tt } \begin{aligned}
& \left\{\begin{array}{l}
\alpha \rightarrow 1, \\
x_{5} \rightarrow x_{4}, \\
\langle\mathbf{2}\rangle,
\end{array}\right. \\
& \text { tt }\left\{\begin{array}{l}
\alpha \rightarrow 2, \\
x_{4} \rightarrow x_{4} x_{5}, \\
\langle\mathbf{2 7}\rangle,
\end{array}\right. \\
& \text { tttt }\left\{\begin{array}{l}
\alpha \rightarrow \gamma+3, \\
x_{4} \rightarrow x_{4} x_{5}, \\
\langle\mathbf{3 1}\rangle,
\end{array}\right.
\end{aligned}
$$

where $\gamma$ is a natural parameter.

Proof. The equation 32 is divided into a collection of equations by means of the conditions $\alpha=0, \alpha=1, \alpha=2, \alpha=\gamma+3$, where $\gamma$ is a natural parameter.

The equation 32 with $\alpha=0$ can be reduced by the transformation t to the equation $x_{1} x_{2}=x_{2} x_{1}$, that is, to $\mathbf{1}$.

The equation 32 with $\alpha=1$ can be reduced by the transformation tt to the equation $x_{1} x_{2} x_{3}=x_{2} x_{3} x_{1}$, that is, to 2 .

The equation 32 with $\alpha=2$ can be reduced by the transformation ttt to the equation $x_{1} x_{2} x_{3} x_{4}=x_{2} x_{3}^{2} x_{1}$, that is, to 27 .

The equation 32 with $\alpha=\gamma+3$ can be reduced by the transformation tttt to the equation $x_{1} x_{2} x_{3} x_{4}=x_{2} x_{3}^{\gamma+3} x_{1}$, that is, to $\mathbf{3 1}$.

Proposition 33. The general solution the equation

33

$$
x_{1} x_{2}=x_{3}^{\alpha} x_{4},
$$

where $\alpha$ is a natural parameter, is described by the transformations

$$
\left\{\begin{array} { l } 
{ x _ { 1 } \rightarrow x _ { 3 } ^ { \alpha } x _ { 4 } , } \\
{ x _ { 4 } \rightarrow x _ { 4 } x _ { 2 } , }
\end{array} \quad \left\{\begin{array} { l } 
{ x _ { 1 } \rightarrow ( x _ { 1 } x _ { 3 } ) ^ { \beta } x _ { 1 } , } \\
{ x _ { 2 } \rightarrow x _ { 3 } ( x _ { 1 } x _ { 3 } ) ^ { \gamma - \beta } x _ { 4 } , } \\
{ x _ { 3 } \rightarrow x _ { 1 } x _ { 3 } , } \\
{ \alpha \rightarrow \gamma + 1 , }
\end{array} \quad \left\{\begin{array}{l}
x_{1} \rightarrow 1, \\
x_{2} \rightarrow x_{4}, \\
\alpha \rightarrow 0,
\end{array}\right.\right.\right.
$$

where $\beta, \gamma$ are natural parameters.

Proof. The equation 33 can be divided by means of the conditions $\partial\left(x_{2}\right) \leq \partial\left(x_{4}\right)$ and $\partial\left(x_{2}\right) \geq \partial\left(x_{4}\right)$.

The equation 33 with $\partial\left(x_{2}\right) \leq \partial\left(x_{4}\right)$ can be reduced by the transformation $x_{4} \rightarrow x_{4} x_{2}$ to the equation $x_{1}=x_{3}^{\alpha} x_{4}$. 
The equation 33 with $\partial\left(x_{2}\right) \geq \partial\left(x_{4}\right)$ and $\alpha>0$ can be reduced by the transformation

$$
\left\{\begin{array}{l}
x_{3} \rightarrow x_{1} x_{3} \\
x_{1} \rightarrow\left(x_{1} x_{3}\right)^{\beta} x_{1} \\
x_{2} \rightarrow x_{3}\left(x_{1} x_{3}\right)^{\alpha-\beta-1} x_{4}
\end{array}\right.
$$

to the equation 1.

The equation 33 with $\partial\left(x_{2}\right) \geq \partial\left(x_{4}\right)$ and $\alpha=0$ can be reduced by the transformation

$$
\left\{\begin{array}{l}
x_{1} \rightarrow 1 \\
x_{2} \rightarrow x_{4} \\
\alpha \rightarrow 0
\end{array}\right.
$$

to the equation 1.

Proposition 34. The general solution of the equation

34

$$
x_{1} x_{2} x_{3} x_{4}=x_{2}^{\alpha+1} x_{5},
$$

where $\alpha$ is a natural parameter, is described by the transformations

$$
\left\{\begin{array} { l } 
{ x _ { 1 } \rightarrow x _ { 2 } ^ { \alpha + 1 } x _ { 1 } , } \\
{ x _ { 5 } \rightarrow x _ { 1 } x _ { 2 } x _ { 3 } x _ { 4 } , }
\end{array} \quad \left\{\begin{array} { l } 
{ x _ { 1 } \rightarrow x _ { 1 } ^ { ( \eta + \theta ) \beta + \eta } , } \\
{ x _ { 2 } \rightarrow x _ { 1 } ^ { \eta + \theta } , } \\
{ \alpha \rightarrow \gamma + 1 , }
\end{array} \quad \left\{\begin{array}{l}
x_{1} \rightarrow\left(x_{1} x_{2}\right)^{\alpha} x_{1}, \\
x_{2} \rightarrow x_{1} x_{2}, \\
x_{5} \rightarrow x_{5} x_{3} x_{4},
\end{array}\right.\right.\right.
$$

where $\beta, \gamma, \eta, \theta$ are natural parameters with $\beta \leq \gamma$.

Proof. The equation $\mathbf{3 4}$ can be divided by means of the conditions $\partial\left(x_{1}\right) \geq \partial\left(x_{2}^{\alpha+1}\right)$ and $\partial\left(x_{1}\right)<\partial\left(x_{2}^{\alpha+1}\right)$.

The equation 33 with $\partial\left(x_{1}\right) \geq \partial\left(x_{2}^{\alpha+1}\right)$ can be reduced by the transformation $x_{1} \rightarrow x_{2}^{\alpha+1} x_{1}$ to the equation $x_{1} x_{2} x_{3} x_{4}=x_{5}$.

The equation 34 with $\partial\left(x_{1}\right)<\partial\left(x_{2}^{\alpha+1}\right)$ can be reduced by the transformation

$$
\begin{aligned}
& x_{1} \rightarrow x_{2}^{\beta} x_{1} \quad(\beta<\alpha+1), \\
& x_{2} \rightarrow x_{1} x_{2}
\end{aligned}
$$

to the equation $E$ :

$$
x_{1} x_{2} x_{3} x_{4}=x_{2}\left(x_{1} x_{2}\right)^{\alpha-\beta} x_{5} \text { with } \beta \leq \alpha, \partial\left(x_{2}\right)>0 .
$$

If $\beta<\alpha$, then by Proposition 1 the equation $E$ is reduced by the parametric transformation

$$
\left\{\begin{array}{l}
x_{1} \rightarrow x_{1}^{\eta}, \\
x_{2} \rightarrow x_{1}^{\theta}
\end{array}\right.
$$

where $\eta, \theta$ are natural parameters, to the equation

$$
x_{3} x_{4}=x_{1}^{\theta+(\eta+\theta)(\alpha-\beta-1)} x_{5}
$$

with $\partial\left(x_{1}^{\theta}\right)>0$, that is, to $\mathbf{3 3}$.

If $\beta=\alpha$, then the equation $E$ is reduced by the parametric transformation $x_{5} \rightarrow x_{5} x_{3} x_{4}$ to the equation $x_{1} x_{2}=x_{2} x_{5}$, that is, to 4 . 
Proposition 35. The general solution of the equation

35

$$
x_{1} x_{2} x_{3} x_{4}=x_{3}\left(x_{2} x_{3}\right)^{\alpha+1} x_{5},
$$

where $\alpha$ is a natural parameter, is described by the transformations

$$
\mathrm{t}\left\{\begin{array}{l}
x_{1} \rightarrow\left(x_{1} x_{3} x_{2}\right)^{\tau} x_{1}, \\
x_{3} \rightarrow x_{1} x_{3}, \\
x_{4} \rightarrow x_{3}\left(x_{2} x_{1} x_{3}\right)^{\alpha-\tau} x_{5},
\end{array}\right.
$$

tt $\left\{\begin{array}{l}x_{1} \rightarrow\left(x_{1} x_{3} x_{2}\right)^{\alpha+1} x_{1}, \\ x_{3} \rightarrow x_{1} x_{3}, \\ x_{5} \rightarrow x_{5} x_{4},\end{array}\right.$

$\langle 6\rangle$,

$\operatorname{ttt}\left\{\begin{array}{l}x_{1} \rightarrow x_{3}\left(x_{1} x_{2} x_{3}\right)^{\tau} x_{1}, \\ x_{2} \rightarrow x_{1} x_{2} \\ x_{4} \rightarrow x_{2} x_{3}\left(x_{1} x_{2} x_{3}\right)^{\alpha-\tau-1} x_{5},\end{array}\right.$

$\operatorname{tttt}\left\{\begin{array}{l}x_{1} \rightarrow x_{3}\left(x_{1} x_{2} x_{3}\right)^{\alpha} x_{1}, \\ x_{2} \rightarrow x_{1} x_{2}, \\ x_{5} \rightarrow x_{5} x_{4},\end{array}\right.$

$\langle 7\rangle$

$\operatorname{ttttt}\left\{\begin{array}{l}x_{1} \rightarrow x_{3}\left(x_{2} x_{3}\right)^{\alpha+1} x_{1}, \\ x_{5} \rightarrow x_{1} x_{2} x_{3} x_{4},\end{array}\right.$

where $\tau$ is a natural parameter.

Proof. The equation $\mathbf{3 5}$ can be divided into the collection of equations

(j) 35 with $\partial\left(\left(x_{2} x_{3}\right)^{\tau}\right) \leq \partial\left(x_{1}\right) \leq \partial\left(x_{3}\left(x_{2} x_{3}\right)^{\tau}\right), \tau \leq \alpha+1$,

(jj) 35 with $\partial\left(x_{3}\left(x_{2} x_{3}\right)^{\tau}\right) \leq \partial\left(x_{1}\right) \leq \partial\left(\left(x_{2} x_{3}\right)^{\tau+1}\right), \tau \leq \alpha$,

(jjj) 35 with $\partial\left(x_{3}\left(x_{2} x_{3}\right)^{\alpha+1}\right) \leq \partial\left(x_{1}\right)$.

The equation $(\mathrm{j})$ can be reduced by the transformation

$$
\begin{aligned}
& x_{1} \rightarrow\left(x_{3} x_{2}\right)^{\tau} x_{1}, \\
& x_{3} \rightarrow x_{1} x_{3}
\end{aligned}
$$

to the equation $E$ :

$$
x_{2} x_{1} x_{3} x_{4}=x_{3}\left(x_{2} x_{1} x_{3}\right)^{\alpha+1-\tau} x_{5} .
$$

If $\tau<\alpha+1$, then the equation $E$ can be reduced by the transformation $x_{4} \rightarrow$ $x_{3}\left(x_{2} x_{1} x_{3}\right)^{\alpha-\tau} x_{5}$ to the equation $x_{2} x_{1} x_{3}=x_{3} x_{2} x_{1}$, that is, to 2 .

If $\tau=\alpha+1$, then the equation $E$ can be reduced by the transformation $x_{5} \rightarrow x_{5} x_{4}$ to the equation $x_{2} x_{1} x_{3}=x_{3} x_{5}$, that is, to 6 .

The equation (jj) can be reduced by the transformation

$$
\begin{aligned}
& x_{1} \rightarrow x_{3}\left(x_{2} x_{3}\right)^{\tau} x_{1}, \\
& x_{2} \rightarrow x_{1} x_{2}
\end{aligned}
$$

to the equation $E$ :

$$
x_{1} x_{2} x_{3} x_{4}=x_{2} x_{3}\left(x_{1} x_{2} x_{3}\right)^{\alpha-\tau} x_{5}
$$


If $\tau<\alpha$, then the equation $E$ can be reduced by the transformation $x_{4} \rightarrow$ $x_{2} x_{3}\left(x_{1} x_{2} x_{3}\right)^{\alpha-\tau-1} x_{5}$ to the equation $x_{1} x_{2} x_{3}=x_{2} x_{3} x_{1}$, that is, to $\mathbf{2}$.

If $\tau=\alpha$, then the equation $E$ can be reduced by the transformation $x_{5} \rightarrow x_{5} x_{4}$ to the equation $x_{1} x_{2} x_{3}=x_{2} x_{3} x_{5}$, that is, to 7 .

The equation (jjj) can be reduced by the transformation

$$
x_{1} \rightarrow x_{3}\left(x_{2} x_{3}\right)^{\alpha+1} x_{1}
$$

to the equation $x_{1} x_{2} x_{3} x_{4}=x_{5}$.

Proposition 36. The general solution of the equation

$$
x_{1} x_{2} x_{3} x_{4}=x_{3}^{\alpha+1} x_{5},
$$

where $\alpha$ is a natural parameter, is described by the transformations

$$
\begin{aligned}
& \mathrm{t}\left\{\begin{array}{l}
x_{1} \rightarrow x_{3}^{\alpha+1} x_{1}, \\
x_{5} \rightarrow x_{1} x_{2} x_{3} x_{4},
\end{array}\right. \\
& \mathrm{tt}\left\{\begin{array}{l}
x_{1} \rightarrow\left(x_{1} x_{3}\right)^{\beta} x_{1}, \\
x_{3} \rightarrow x_{1} x_{3},
\end{array}\right. \\
& \mathrm{ttt}\left\{\begin{array}{l}
\langle\mathbf{3 5}\rangle, \\
x_{3} \rightarrow x_{1} x_{3}, \\
x_{5} \rightarrow x_{5} x_{4},
\end{array}\right.
\end{aligned}
$$

$\langle 6\rangle$,

where $\beta$ is a natural parameter with $\beta<\alpha$.

Proof. The equation $\mathbf{3 6}$ can be divided by means of the conditions $\partial\left(x_{1}\right) \geq \partial\left(x_{3}^{\alpha+1}\right)$ and $\partial\left(x_{1}\right) \leq \partial\left(x_{3}^{\alpha+1}\right)$.

The equation 36 with $\partial\left(x_{1}\right) \geq \partial\left(x_{3}^{\alpha+1}\right)$ can be reduced by the transformation $x_{1} \rightarrow x_{3}^{\alpha+1} x_{1}$ to the equation $x_{1} x_{2} x_{3} x_{4}=x_{5}$.

The equation 36 with $\partial\left(x_{1}\right) \leq \partial\left(x_{3}^{\alpha+1}\right)$ can be reduced by the transformation

$$
\begin{aligned}
& x_{1} \rightarrow x_{3}^{\beta} x_{1} \quad(\beta<\alpha+1), \\
& x_{3} \rightarrow x_{1} x_{3}
\end{aligned}
$$

to the equation $E$ :

$$
x_{2} x_{1} x_{3} x_{4}=x_{3}\left(x_{1} x_{3}\right)^{\alpha-\beta} x_{5} \quad \text { with } \beta \leq \alpha .
$$

If $\beta<\alpha$, then $E$ is $\mathbf{3 5}$.

If $\beta=\alpha$, then $E$ can be reduced by the transformation $x_{5} \rightarrow x_{5} x_{4}$ to the equation $x_{2} x_{1} x_{3}=x_{3} x_{5}$, that is, to 6 .

Proposition 37. The general solution of the equation

37

$$
x_{1} x_{2} x_{3} x_{4}=x_{2} x_{3}^{\alpha+1} x_{5},
$$

where $\alpha$ is a natural parameter, is described by the transformations

$$
\mathrm{t}\left\{\begin{array} { r l } 
{ x _ { 1 } \rightarrow 1 , } \\
{ x _ { 4 } \rightarrow x _ { 3 } ^ { \alpha } x _ { 5 } , }
\end{array} \quad \text { tt } \left\{\begin{array}{rl}
x_{1} \rightarrow & x_{2} x_{1}, \\
x_{2} \rightarrow & \left(x_{2} x_{1}\right)^{\lambda} x_{2}, \\
& \langle\mathbf{3 6}\rangle,
\end{array}\right.\right.
$$

where $\lambda$ is a natural parameter. 
Proof. The equation $\mathbf{3 7}$ can be divided by means of the conditions $\partial\left(x_{1}\right)=0$ and $\partial\left(x_{1}\right)>0$.

The equation 37 with $\partial\left(x_{1}\right)=0$ can be reduced by the transformation $x_{1} \rightarrow 1$ to the equation $x_{4}=x_{3}^{\alpha} x_{5}$.

The equation 37 with $\partial\left(x_{1}\right)>0$ by Proposition 8 can be reduced by the transformation $x_{2} \rightarrow x_{1}^{\lambda} x_{2}$ to the equation 37 with $\partial\left(x_{1}\right)>\partial\left(x_{2}\right)$, which in turn can be reduced by the transformation $x_{1} \rightarrow x_{2} x_{1}$ to the equation

$$
x_{1} x_{2} x_{3} x_{4}=x_{3}^{\alpha+1} x_{5} \quad \text { with } \partial\left(x_{1}\right)>0,
$$

that is, to $\mathbf{3 6}$ with $\partial\left(x_{1}\right)>0$. On the other hand, the transformation tt, $\langle\mathbf{3 6}\rangle$ is a parametric solution of $\mathbf{3 7}$.

Proposition 38. The general solution of the equation

38

$$
x_{2} x_{1} x_{3} x_{4}=x_{3} x_{1}\left(\left(x_{1} x_{3}\right)^{\lambda+1} x_{1}\right)^{\kappa+1} x_{5},
$$

where $\lambda, \kappa$ are natural parameters, is described by the transformations

$$
\begin{aligned}
& \mathrm{t} 1\left\{\begin{array}{l}
x_{3} \rightarrow x_{2} x_{3}, \\
x_{4} \rightarrow x_{4} x_{3}\left(x_{1} x_{2} x_{3}\right)^{\lambda} x_{1}\left(\left(x_{1} x_{2} x_{3}\right)^{\lambda+1} x_{1}\right)^{\kappa} x_{5},
\end{array}\right. \\
& \langle\mathbf{1 2}\rangle \text {, } \\
& \mathrm{t} 2\left\{\begin{array}{l}
x_{1} \rightarrow x_{1}^{\eta+\theta}, \\
x_{2} \rightarrow x_{3} x_{1}^{\theta}, \\
x_{4} \rightarrow x_{4}\left(x_{1}^{\eta+\theta} x_{3}\right)^{\lambda} x_{1}^{\eta+\theta}\left(\left(x_{1}^{\eta+\theta} x_{3}\right)^{\lambda+1} x_{1}^{\eta+\theta}\right)^{\kappa} x_{5},
\end{array}\right. \\
& \mathrm{t} 3\left\{\begin{array}{l}
x_{1} \rightarrow x_{2} x_{1} \\
x_{2} \rightarrow x_{3} x_{2} x_{1}\left(\left(x_{1} x_{2} x_{3}\right)^{\lambda+1} x_{2} x_{1}\right)^{\tau}\left(x_{2} x_{1} x_{3}\right)^{\sigma} x_{2}, \\
x_{4} \rightarrow x_{1}\left(x_{2} x_{3} x_{1}\right)^{\lambda-\sigma}\left(\left(x_{2} x_{1} x_{3}\right)^{\lambda+1} x_{2} x_{1}\right)^{\kappa-\tau} x_{5}
\end{array}\right. \\
& \langle\mathbf{2}\rangle, \\
& \mathrm{t} 4\left\{\begin{array}{l}
x_{1} \rightarrow x_{1}^{\eta+\theta}, \\
x_{2} \rightarrow x_{3} x_{1}^{\eta+\theta}\left(\left(x_{1}^{\eta+\theta} x_{3}\right)^{\lambda+1} x_{1}^{\eta+\theta}\right)^{\tau}\left(x_{1}^{\eta+\theta} x_{3}\right)^{\lambda+1} x_{1}^{\theta}, \\
x_{4} \rightarrow x_{4} x_{1}^{\eta+\theta}\left(x_{3} x_{1}^{\eta+\theta}\right)^{\lambda}\left(\left(x_{1}^{\eta+\theta} x_{3}\right)^{\lambda+1} x_{1}^{\eta+\theta}\right)^{\kappa-\tau-1} x_{5},
\end{array}\right. \\
& \langle\mathbf{5}\rangle, \\
& \text { t5 }\left\{\begin{aligned}
& x_{1} \rightarrow\left(x_{1} x_{2}\right)^{\beta+1} x_{1}, \\
& x_{2} \rightarrow x_{3}\left(x_{1} x_{2}\right)^{\beta} x_{1}\left(\left(\left(x_{1} x_{2}\right)^{\beta} x_{1} x_{3}\right)^{\lambda+1}\left(x_{1} x_{2}\right)^{\beta} x_{1}\right)^{\kappa} \\
& \cdot\left(\left(x_{1} x_{2}\right)^{\beta} x_{1} x_{3}\right)^{\lambda+1} x_{1} x_{2}, \\
& x_{5} \rightarrow x_{2} x_{1} x_{3} x_{4}
\end{aligned}\right. \\
& \mathrm{t} 6\left\{\begin{array}{l}
x_{2} \rightarrow x_{2} x_{3} x_{1}\left(\left(x_{1} x_{2} x_{3}\right)^{\lambda+1} x_{1}\right)^{\tau}\left(x_{1} x_{2} x_{3}\right)^{\sigma} x_{1} x_{2} \\
x_{3} \rightarrow x_{2} x_{3} \\
x_{4} \rightarrow x_{3} x_{1}\left(x_{2} x_{3} x_{1}\right)^{\lambda-\sigma-1}\left(\left(x_{1} x_{2} x_{3}\right)^{\lambda+1} x_{1}\right)^{\kappa-\tau} x_{5}
\end{array}\right.
\end{aligned}
$$




$$
\mathrm{t} 7\left\{\begin{array}{l}
x_{2} \rightarrow x_{2} x_{3} x_{1}\left(\left(x_{1} x_{2} x_{3}\right)^{\lambda+1} x_{1}\right)^{\tau}\left(x_{1} x_{2} x_{3}\right)^{\lambda} x_{1} x_{2}, \\
x_{3} \rightarrow x_{2} x_{3} \\
x_{4} \rightarrow x_{4} x_{3}\left(x_{1} x_{2} x_{3}\right)^{\lambda} x_{1}\left(\left(x_{1} x_{2} x_{3}\right)^{\lambda+1} x_{1}\right)^{\kappa-\tau-1} x_{5},
\end{array}\right.
$$

$\mathrm{t} 8\left\{\begin{array}{l}x_{2} \rightarrow x_{2} x_{3} x_{1}\left(\left(x_{1} x_{2} x_{3}\right)^{\lambda+1} x_{1}\right)^{\kappa}\left(x_{1} x_{2} x_{3}\right)^{\lambda} x_{1} x_{2}, \\ x_{3} \rightarrow x_{2} x_{3} \\ x_{5} \rightarrow x_{5} x_{4}\end{array}\right.$

$\langle\mathbf{1 0}\rangle$,

$$
\text { t9 }\left\{\begin{array}{l}
x_{2} \rightarrow x_{3} x_{1}\left(\left(x_{1} x_{3}\right)^{\lambda+1} x_{1}\right)^{\kappa+1} x_{2} \\
x_{5} \rightarrow x_{2} x_{1} x_{3} x_{4}
\end{array}\right.
$$

where $\eta, \theta, \tau, \sigma, \beta$ are natural parameters.

Proof. The equation $\mathbf{3 8}$ can be divided into the collection of equations:

(j) 38 with $\partial\left(x_{2}\right)<\partial\left(x_{3}\right)$,

(jj) 38 with $\partial\left(x_{3}\right) \leq \partial\left(x_{2}\right) \leq \partial\left(x_{3} x_{1}\right)$,

(jjj) 38 with

$$
\begin{aligned}
& \partial\left(x_{3} x_{1}\left(\left(x_{1} x_{3}\right)^{\lambda+1} x_{1}\right)^{\tau}\left(x_{1} x_{3}\right)^{\sigma} \leq \partial\left(x_{2}\right)\right. \\
& \quad \leq \partial\left(x_{3} x_{1}\left(\left(x_{1} x_{3}\right)^{\lambda+1} x_{1}\right)^{\tau}\left(x_{1} x_{3}\right)^{\sigma} x_{1}\right), \quad \tau<\kappa+1, \sigma \leq \lambda+1,
\end{aligned}
$$

(jjjj) 38 with

$$
\begin{aligned}
& \partial\left(x_{3} x_{1}\left(\left(x_{1} x_{3}\right)^{\lambda+1} x_{1}\right)^{\tau}\left(x_{1} x_{3}\right)^{\sigma} x_{1}\right) \leq \partial\left(x_{2}\right) \\
& <\partial\left(x_{3} x_{1}\left(\left(x_{1} x_{3}\right)^{\lambda+1} x_{1}\right)^{\tau}\left(x_{1} x_{3}\right)^{\sigma+1}\right), \quad \tau<\kappa+1, \sigma<\lambda+1,
\end{aligned}
$$

(jjjjj) 38 with $\partial\left(x_{3} x_{1}\left(\left(x_{1} x 3\right)^{\lambda+1} x_{1}\right)^{\kappa+1}\right) \leq \partial\left(x_{2}\right)$.

The equation $(\mathrm{j})$ can be reduced by the transformation

$$
\begin{aligned}
& x_{3} \rightarrow x_{2} x_{3}, \\
& x_{4} \rightarrow x_{4} x_{3}\left(x_{1} x_{2} x_{3}\right)^{\lambda} x_{1}\left(\left(x_{1} x_{2} x_{3}\right)^{\lambda+1} x_{1}\right)^{\kappa} x_{5}
\end{aligned}
$$

to the equation $x_{1} x_{2} x_{3} x_{4}=x_{3} x_{1}^{2} x_{2}$ with $\partial\left(x_{3}\right)>0$, that is, to $\mathbf{1 2}$.

The equation (jj) can be reduced by the transformation

$$
\begin{gathered}
x_{2} \rightarrow x_{3} x_{2}, \\
x_{1} \rightarrow x_{2} x_{1}, \\
\left\{\begin{array}{l}
x_{1} \rightarrow x_{1}^{\eta}, \\
x_{2} \rightarrow x_{1}^{\theta},
\end{array}\right. \\
x_{4} \rightarrow x_{4}\left(x_{1}^{\eta+\theta} x_{3}\right)^{\lambda} x_{1}^{\eta+\theta}\left(\left(x_{1}^{\eta+\theta} x_{3}\right)^{\lambda+1} x_{1}^{\eta+\theta}\right)^{\kappa} x_{5}
\end{gathered}
$$

to the equation $x_{3} x_{4}=x_{1}^{\eta} x_{3}$, that is, $\left.\left(x_{2} x_{3} x_{1}\right)^{\beta+1} x_{2}\right)^{\kappa}$ to $\mathbf{5}$.

The equation (jjj) can be reduced by the transformation

$$
\begin{aligned}
& x_{2} \rightarrow x_{3} x_{1}\left(\left(x_{1} x_{3}\right)^{\lambda+1} x_{1}\right)^{\tau}\left(x_{1} x_{3}\right)^{\sigma} x_{2}, \\
& x_{1} \rightarrow x_{2} x_{1}
\end{aligned}
$$

to the equation $E$ :

$$
x_{2} x_{1} x_{3} x_{4}=x_{1}\left(x_{3} x_{2} x_{1}\right)^{\lambda+1-\sigma}\left(\left(x_{2} x_{1} x_{3}\right)^{\lambda+1} x_{2} x_{1}\right)^{\kappa-\tau} x_{5} .
$$


The equation $E$ with $\lambda+1>\sigma$ can be reduced by the transformation

$$
x_{4} \rightarrow x_{1}\left(x_{2} x_{3} x_{1}\right)^{\lambda-\sigma}\left(\left(x_{2} x_{1} x_{3}\right)^{\lambda+1} x_{2} x_{1}\right)^{\kappa-\tau} x_{5}
$$

to the equation $x_{2} x_{1} x_{3}=x_{1} x_{3} x_{2}$, that is, to $\mathbf{2}$.

The equation $E$ with $\lambda+1=\sigma$ and $\tau<\kappa$ can be reduced by the transformation

$$
\begin{gathered}
\left\{\begin{array}{l}
x_{1} \rightarrow x_{1}^{\eta}, \\
x_{2} \rightarrow x_{1}^{\theta},
\end{array}\right. \\
x_{4} \rightarrow x_{4} x_{1}^{\eta+\theta}\left(x_{3} x_{1}^{\eta+\theta}\right)^{\lambda}\left(\left(x_{1}^{\eta+\theta} x_{3}\right)^{\lambda+1} x_{1}^{\eta+\theta}\right)^{\kappa-\tau-1} x_{5}
\end{gathered}
$$

to the equation $x_{3} x_{4}=x_{1}^{\eta} x_{3}$, that is, $\mathbf{5}$.

The equation $E$ with $\lambda+1=\sigma$ and $\tau=\kappa$ can be reduced by the transformation

$$
\begin{aligned}
& x_{1} \rightarrow x_{2}^{\beta} x_{1}, \\
& x_{2} \rightarrow x_{1} x_{2}, \\
& x_{5} \rightarrow x_{2} x_{1} x_{3} x_{4}
\end{aligned}
$$

to 1 .

The equation (jjjj) can be reduced by the transformation

$$
\begin{aligned}
& x_{2} \rightarrow x_{3} x_{1}\left(\left(x_{1} x_{3}\right)^{\lambda+1} x_{1}\right)^{\tau}\left(x_{1} x_{3}\right)^{\sigma} x_{1} x_{2}, \\
& x_{3} \rightarrow x_{2} x_{3}
\end{aligned}
$$

to the equation $E$ :

$$
x_{1} x_{2} x_{3} x_{4}=x_{3} x_{1}\left(x_{2} x_{3} x_{1}\right)^{\lambda-\sigma}\left(\left(x_{1} x_{2} x_{3}\right)^{\lambda+1} x_{1}\right)^{\kappa-\tau} x_{5}
$$

with $\partial\left(x_{3}\right)>0$.

The equation $E$ with $\lambda>\sigma$ can be reduced by the transformation

$$
x_{4} \rightarrow x_{3} x_{1}\left(x_{2} x_{3} x_{1}\right)^{\lambda-\sigma-1}\left(\left(x_{1} x_{2} x_{3}\right)^{\lambda+1} x_{1}\right)^{\kappa-\tau} x_{5}
$$

to the equation $x_{2} x_{1} x_{3}=x_{1} x_{3} x_{2}$, that is, to 2 with $\partial\left(x_{3}\right)>0$. On the other hand, t6 is a parametric solution of $\mathbf{3 8}$.

The equation $E$ with $\lambda=\sigma$ and $\kappa>\tau$ can be reduced by the transformation

$$
x_{4} \rightarrow x_{4} x_{3}\left(x_{1} x_{2} x_{3}\right)^{\lambda} x_{1}\left(\left(x_{1} x_{2} x_{3}\right)^{\lambda+1} x_{1}\right)^{\kappa-\tau-1} x_{5}
$$

to the equation $x_{1} x_{2} x_{3} x_{4}=x_{3} x_{1}^{2} x_{2}$ with $\partial\left(x_{3}\right)>0$, that is, to $\mathbf{1 2}$.

The equation $E$ with $\lambda=\sigma$ and $\kappa=\tau$ can be reduced by the transformation $x_{5} \rightarrow x_{5} x_{4}$ to the equation $x_{1} x_{2} x_{3}=x_{3} x_{1} x_{5}$ with $\partial\left(x_{3}\right)>0$, that is, to 10 with $\partial\left(x_{3}\right)>0$. On the other hand, t8 is a parametric solution of $\mathbf{3 8}$.

The equation (jjjjj) can be reduced by the transformation

$$
\begin{aligned}
x_{2} & \rightarrow x_{3} x_{1}\left(\left(x_{1} x_{3}\right)^{\lambda+1} x_{1}\right)^{\kappa+1} x_{2}, \\
x_{5} & \rightarrow x_{2} x_{1} x_{3} x_{4}
\end{aligned}
$$

to the equation 1.

Proposition 39. The general solution of the equation

$$
x_{1} x_{2} x_{3} x_{4}=x_{3} x_{1}^{\alpha+1} x_{5},
$$


where $\alpha$ is a natural parameter, is described by the transformations

$$
\begin{aligned}
& \mathrm{t} 1\left\{\begin{array} { l } 
{ x _ { 1 } \rightarrow 1 , } \\
{ x _ { 2 } \rightarrow 1 , } \\
{ x _ { 4 } \rightarrow x _ { 5 } , }
\end{array} \quad \mathrm { t } 2 \left\{\begin{array}{c}
x_{2} \rightarrow x_{3} x_{2}, \\
x_{3} \rightarrow\left(x_{1} x_{3} x_{2}\right)^{\lambda} x_{1} x_{3}, \\
\langle\mathbf{3 4}\rangle,
\end{array}\right.\right. \\
& \text { t3 }\left\{\begin{array}{l}
x_{1} \rightarrow\left(x_{1} x_{3}\right)^{\gamma+1} x_{1} \\
x_{3} \rightarrow\left(\left(x_{1} x_{3}\right)^{\gamma+1} x_{1} x_{2}\right)^{\lambda} x_{1} x_{3} \\
\alpha \rightarrow \kappa+1
\end{array}\right. \\
& \langle 38\rangle, \\
& \mathrm{t} 4\left\{\begin{array}{l}
x_{1} \rightarrow\left(x_{1} x_{3}\right)^{\gamma+1} x_{1}, \\
x_{2} \rightarrow x_{3} x_{2}, \\
x_{3} \rightarrow\left(\left(x_{1} x_{3}\right)^{\gamma+2} x_{2}\right)^{\lambda} x_{1} x_{3}, \\
x_{5} \rightarrow x_{5} x_{3} x_{4}, \\
\alpha \rightarrow 0,
\end{array}\right. \\
& \langle 4\rangle, \\
& \text { t5 }\left\{\begin{array}{l}
x_{1} \rightarrow\left(x_{1} x_{2} x_{3}\right)^{\gamma+1} x_{1} \\
x_{3} \rightarrow\left(\left(x_{1} x_{2} x_{3}\right)^{\gamma+1} x_{1} x_{2}\right)^{\lambda} x_{1} x_{2} x_{3} \\
x_{5} \rightarrow x_{5} x_{4} \\
\alpha \rightarrow 0
\end{array}\right. \\
& \langle\mathbf{1 0}\rangle, \\
& \mathrm{t} 7\left\{\begin{array} { l } 
{ x _ { 2 } \rightarrow 1 , } \\
{ x _ { 3 } \rightarrow x _ { 1 } ^ { \lambda } , } \\
{ x _ { 4 } \rightarrow x _ { 5 } , } \\
{ \alpha \rightarrow 0 , }
\end{array} \quad \mathrm { t } 8 \quad \left\{\begin{array}{l}
x_{1} \rightarrow x_{2} x_{1}, \\
x_{2} \rightarrow\left(x_{2} x_{1}\right)^{\gamma} x_{2}, \\
x_{3} \rightarrow\left(\left(x_{2} x_{1}\right)^{\gamma+1} x_{2}\right)^{\lambda}, \\
x_{4} \rightarrow\left(x_{1} x_{2}\right)^{\beta-\gamma} x_{1} x_{5}, \\
\alpha \rightarrow \beta+1,
\end{array}\right.\right. \\
& \mathrm{t} 6\left\{\begin{array}{l}
x_{2} \rightarrow x_{1}^{\alpha} x_{5} \\
x_{3} \rightarrow\left(x_{1}^{\alpha+1} x_{5}\right)^{\lambda} \\
x_{5} \rightarrow x_{5} x_{4}
\end{array}\right.
\end{aligned}
$$

where $\lambda, \gamma, \kappa, \beta$ are natural parameters.

Proof. The equation 39 can be divided into the collection of equations:

(j) 39 with $\partial\left(x_{1} x_{2}\right)=0$,

(jj) 39 with $\partial\left(x_{1} x_{2}\right)>0$.

The equation $(\mathrm{j})$ can be reduced by the transformation $x_{1} \rightarrow 1, x_{2} \rightarrow 1$ to the equation $x_{4}=x_{5}$.

The equation (jj) by Proposition 8 can be reduced by the transformation

$$
x_{3} \rightarrow\left(x_{1} x_{2}\right)^{\lambda} x_{3},
$$

where $\lambda$ is a natural parameter, to the equation $E$ :

$$
x_{1} x_{2} x_{3} x_{4}=x_{3} x_{1}^{\alpha+1} x_{5} \quad \text { with } \partial\left(x_{3}\right)<\partial\left(x_{1} x_{2}\right) .
$$

The equation $E$ can be divided by mean of the conditions $\partial\left(x_{3}\right) \geq \partial\left(x_{1}\right)$ and $\partial\left(x_{1}\right) \geq \partial\left(x_{3}\right)$.

The equation $E$ with $\partial\left(x_{3}\right) \geq \partial\left(x_{1}\right)$ can be reduced by the transformation $x_{3} \rightarrow$ $x_{1} x_{3}, x_{2} \rightarrow x_{3} x_{2}$ to the equation $x_{2} x_{1} x_{3} x_{4}=x_{1}^{\alpha+1} x_{5}$ with $\partial\left(x_{2}\right)>0$, that is, to 34 with $\partial\left(x_{1}\right)>0$. On the other hand, t2 is a parametric solution of $\mathbf{3 9}$.

The equation $E$ with $\partial\left(x_{1}\right) \geq \partial\left(x_{3}\right)$ can be reduced by the transformation $x_{1} \rightarrow$ $x_{3} x_{1}$ to the equation $E_{1}$ :

$$
x_{1} x_{2} x_{3} x_{4}=\left(x_{3} x_{1}\right)^{\alpha+1} x_{5} \quad \text { with } \partial\left(x_{1} x_{2}\right)>0 .
$$

The equation $E_{1}$ with $\partial\left(x_{3}\right)>0$ by Proposition 8 can be reduced by the transformation $x_{1} \rightarrow x_{3}^{\gamma} x_{1}, x_{3} \rightarrow x_{1} x_{3}$ to the equation $E_{2}$ :

$$
x_{2} x_{1} x_{3} x_{4}=x_{3} x_{1}\left(\left(x_{1} x_{3}\right)^{\gamma+1} x_{1}\right)^{\alpha} x_{5} \quad \text { with } \partial\left(x_{3}\right)>0 .
$$


The equation $E_{2}$ with $\alpha>0$ is $\mathbf{3 8}$ with $\partial\left(x_{3}\right)>0$. On the other hand, t3 is a parametric solution of $\mathbf{3 9}$.

The equation $E_{2}$ with $\alpha=0$ and $\partial\left(x_{2}\right) \geq \partial\left(x_{3}\right)$ can be reduced by the transformation $x_{2} \rightarrow x_{3} x_{2}, x_{5} \rightarrow x_{5} x_{3} x_{4}$ to the equation $x_{2} x_{1}=x_{1} x_{5}$, that is, to 4 .

The equation $E_{2}$ with $\alpha=0$ and $\partial\left(x_{2}\right) \leq \partial\left(x_{3}\right)$ can be reduced by the transformation $x_{3} \rightarrow x_{2} x_{3}$ to the equation $x_{1} x_{2} x_{3}=x_{3} x_{1} x_{5}$, that is, to 10 .

The equation $E_{1}$ with $\partial\left(x_{3}\right)=0$ can be reduced by the transformation $x_{3} \rightarrow 1$ the equation $E_{3}$ :

$$
x_{2} x_{4}=x_{1}^{\alpha} x_{5}
$$

The equation $E_{3}$ with $\partial\left(x_{4}\right) \leq \partial\left(x_{5}\right)$ can be reduced by the transformation $x_{5} \rightarrow x_{5} x_{4}$ to the equation $x_{2}=x_{1}^{\alpha} x_{5}$.

The equation $E_{3}$ with $\partial\left(x_{4}\right) \geq \partial\left(x_{5}\right)$ and $\alpha=0$ can be reduced by the transformation $x_{4}=x_{4} x_{5}, \alpha \rightarrow 0$ to the equation $x_{2} x_{4}=1$.

The equation $E_{3}$ with $\partial\left(x_{4}\right) \geq \partial\left(x_{5}\right)$ and $\alpha=\beta+1$ can be reduced by the transformation

$$
\begin{aligned}
\alpha & \rightarrow \beta+1, \\
x_{1} & \rightarrow x_{2} x_{1}, \\
x_{2} & \rightarrow\left(x_{2} x_{1}\right)^{\gamma} x_{2}, \\
x_{4} & \rightarrow\left(x_{1} x_{2}\right)^{\beta-\gamma} x_{1} x_{5}
\end{aligned}
$$

to the equation $\mathbf{1}$.

\section{More EqUATIONS AND SOlutions}

In this section we will denote the words in the alphabet of primitive parametric words $P_{1}, \ldots, P_{k}$ by $\zeta\left(P_{1}, \ldots, P_{k}\right), v\left(P_{1}, \ldots, P_{k}\right), \varphi\left(P_{1}, \ldots, P_{k}\right)$. The natural numbers will be denoted by $q, r, s$, the natural parameters by $\alpha, \beta, \lambda, \tau$, and the primitive parametric word by $P$.

The proofs of Propositions 40-55 are simple and very similar to proofs of the previous propositions, so we shall omit them.

Proposition 40. The general solution of the equation

40

$$
x_{1} x_{2} x_{3} x_{4}=x_{2} \zeta\left(x_{1} x_{2}, x_{3}\right) x_{1} \quad \text { with } \partial\left(x_{2}\right)>0,
$$

where $x_{3}$ occurs in $\zeta\left(x_{1} x_{2}, x_{3}\right)$, is described by the transformations

$$
\left\{\begin{array}{l}
x_{1} \rightarrow x_{1}^{\alpha}, \\
x_{2} \rightarrow x_{1}^{\beta}, \\
x_{4} \rightarrow x_{4} v\left(x_{1}^{\alpha+\beta}, x_{3}\right) x_{1}^{\alpha},
\end{array}\right.
$$

$\langle\mathbf{5}\rangle$,

where $\zeta\left(x_{1} x_{2}, x_{3}\right)$ is $\left(x_{1} x_{2}\right)^{q+1} x_{3} v\left(x_{1} x_{2}, x_{3}\right)$;

$$
x_{4} \rightarrow x_{4} x_{2} v\left(x_{1} x_{2}, x_{3}\right) x_{1},
$$

$\langle 32\rangle$,

where $\zeta\left(x_{1} x_{2}, x_{3}\right)$ is $x_{3}^{q+1} x_{1} x_{2} v\left(x_{1} x_{2}, x_{3}\right)$; and

where $\zeta\left(x_{1} x_{2}, x_{3}\right)$ is $x_{3}^{q+1}$. 
Proposition 41. The general solution of the equation

41

$$
x_{2} x_{1} x_{3} x_{4}=x_{3} \zeta\left(x_{1} x_{3}, x_{2}\right) x_{1} \quad \text { with } \partial\left(x_{3}\right)>0,
$$

where $x_{2}$ occurs in $\zeta\left(x_{1} x_{3}, x_{2}\right)$, is described by the transformations

$$
x_{4} \rightarrow x_{4} v\left(x_{1} x_{3}, x_{2}\right) x_{1},
$$

where $\zeta\left(x_{1} x_{3}, x_{2}\right)$ is $\left(x_{1} x_{3}\right)^{q+1} x_{2} v\left(x_{1} x_{3}, x_{2}\right)$;

$$
x_{4} \rightarrow x_{4} x_{3} v\left(x_{1} x_{3}, x_{2}\right),
$$

$\langle\mathbf{2 0}\rangle$,

where $\zeta\left(x_{1} x_{3}, x_{2}\right)$ is $x_{2}^{r+1} x_{1} x_{3} v\left(x_{1} x_{3}, x_{2}\right)$;

$$
x_{4} \rightarrow x_{4} x_{3} v\left(x_{1} x_{3}, x_{2}\right),
$$

$\langle\mathbf{1 2}\rangle$,

where $\zeta\left(x_{1} x_{3}, x_{2}\right)$ is $x_{2}^{2} x_{1} x_{3} v\left(x_{1} x_{3}, x_{2}\right)$;

$$
x_{4} \rightarrow x_{4} x_{3} v\left(x_{1} x_{3}, x_{2}\right),
$$

where $\zeta\left(x_{1} x_{3}, x_{2}\right)$ is $x_{2} x_{1} x_{3} v\left(x_{1} x_{3}, x_{2}\right)$;

where $\zeta\left(x_{1} x_{3}, x_{2}\right)$ is $x_{2}^{r+3}$;

where $\zeta\left(x_{1} x_{3}, x_{2}\right)$ is $x_{2}^{2}$; and

where $\zeta\left(x_{1} x_{3}, x_{2}\right)$ is $x_{2}$.

Proposition 42. The general solution of the equation

42

$$
x_{1} x_{2} x_{3} x_{4}=\zeta\left(x_{2}, x_{3}\right) x_{1}
$$

where $x_{2}$ and $x_{3}$ occur in $\zeta\left(x_{2}, x_{3}\right)$, is described by the transformations

$$
\begin{aligned}
& \left\{\begin{array}{l}
x_{2} \rightarrow 1, \\
x_{3} \rightarrow 1, \\
x_{4} \rightarrow 1 ;
\end{array}\right. \\
& x_{1} \rightarrow\left(\varphi\left(x_{2}, x_{3}\right) x_{2} v\left(x_{2}, x_{3}\right)\right)^{\alpha} \varphi\left(x_{2}, x_{3}\right) x_{1}, \\
& x_{2} \rightarrow x_{1} x_{2},
\end{aligned}
$$

$\langle 40\rangle$,

where $\zeta\left(x_{2}, x_{3}\right)$ is $\varphi\left(x_{2}, x_{3}\right) x_{2} v\left(x_{2}, x_{3}\right)$; and

$$
\begin{aligned}
& x_{1} \rightarrow\left(\varphi\left(x_{2}, x_{3}\right) x_{3} v\left(x_{2}, x_{3}\right)\right)^{\alpha} \varphi\left(x_{2}, x_{3}\right) x_{1}, \\
& x_{3} \rightarrow x_{1} x_{3},
\end{aligned}
$$

$\langle 41\rangle$,

where $\zeta\left(x_{2}, x_{3}\right)$ is $\varphi\left(x_{2}, x_{3}\right) x_{3} v\left(x_{2}, x_{3}\right)$. 
Proposition 43. The general solution of the equation

43

$$
x_{1} x_{2} x_{3} x_{4}=x_{2} \zeta\left(x_{1} x_{2}, x_{3}\right) x_{5} \quad \text { with } \partial\left(x_{2}\right)>0
$$

is described by the transformations

where $\zeta\left(x_{1} x_{2}, x_{3}\right)$ is 1 ;

$$
\left\{\begin{array}{l}
x_{1} \rightarrow x_{1}^{\alpha}, \\
x_{2} \rightarrow x_{1}^{\beta},
\end{array}\right.
$$

$\langle\mathbf{3 3}\rangle$,

where $\zeta\left(x_{1} x_{2}, x_{3}\right)$ is $\left(x_{1} x_{2}\right)^{q+1}$;

$$
x_{4} \rightarrow x_{4} x_{2} v\left(x_{1} x_{2}, x_{3}\right) x_{5},
$$

$\langle 40\rangle$,

where $\zeta\left(x_{1} x_{2}, x_{3}\right)$ is $\left(x_{1} x_{2}\right)^{q} x_{3}^{r+1} x_{1} x_{2} v\left(x_{1} x_{2}, x_{3}\right) x_{5}$;

$\langle 37\rangle$,

where $\zeta\left(x_{1} x_{2}, x_{3}\right)$ is $x_{3}^{r+1}$; and

$$
\begin{aligned}
& \left\{\begin{array}{l}
x_{1} \rightarrow x_{1}^{\alpha}, \\
x_{2} \rightarrow x_{1}^{\beta},
\end{array}\right. \\
& x_{4} \rightarrow x_{4} x_{3}^{r} x_{5},
\end{aligned}
$$

where $\zeta\left(x_{1}, x_{2}\right)$ is $\left(x_{1} x_{2}\right)^{q+1} x_{3}^{r+1}$.

Proposition 44. The general solution of the equation

44

$$
x_{2} x_{1} x_{3} x_{4}=x_{3} \zeta\left(x_{1} x_{3}, x_{2}\right) x_{5} \quad \text { with } \partial\left(x_{3}\right)>0
$$

is described by the transformations

$\langle 36\rangle$,

where $\zeta\left(x_{1} x_{3}, x_{2}\right)$ is 1 ;

where $\zeta\left(x_{1} x_{3}, x_{2}\right)$ is $\left(x_{1} x_{3}\right)^{q+1}$;

where $\zeta\left(x_{1} x_{3}, x_{2}\right)$ is $x_{2}^{r+1}$;

$$
x_{4} \rightarrow x_{4} v\left(x_{1} x_{3}, x_{2}\right) x_{5},
$$

$\langle\mathbf{2 9}\rangle$,

where $\zeta\left(x_{1} x_{3}, x_{2}\right)$ is $\left(x_{1} x_{3}\right)^{q+1} x_{2} v\left(x_{1} x_{3}, x_{2}\right)$;

$$
x_{4} \rightarrow x_{4} x_{3} v\left(x_{1} x_{3}, x_{2}\right) x_{5},
$$

where $\zeta\left(x_{1} x_{3}, x_{2}\right)$ is $x_{2} x_{1} x_{3} v\left(x_{1} x_{3}, x_{2}\right) x_{5}$;

$\langle\mathbf{1 2}\rangle$,

where $\zeta\left(x_{1} x_{3}, x_{2}\right)$ is $x_{2}^{2} x_{1} x_{3} v\left(x_{1} x_{3}, x_{2}\right) x_{5}$; and

$\langle\mathbf{2 0}\rangle$, 
where $\zeta\left(x_{1} x_{3}, x_{2}\right)$ is $x_{2}^{s+3} x_{1} x_{3} v\left(x_{1} x_{3}, x_{2}\right) x_{5}$.

Proposition 45. The general solution of the equation

45

$$
x_{1} x_{2} x_{3} x_{4}=\zeta\left(x_{2}, x_{3}\right) x_{5}
$$

where $x_{2}, x_{3}$ occur in $\zeta\left(x_{2}, x_{3}\right)$ is described by the transformations

$$
\begin{gathered}
\left\{\begin{aligned}
x_{1} & \rightarrow \zeta\left(x_{2}, x_{3}\right) x_{1}, \\
x_{5} & \rightarrow x_{1} x_{2} x_{3} x_{4} ;
\end{aligned}\right. \\
x_{1} \rightarrow \varphi\left(x_{2}, x_{3}\right) x_{1}, \\
x_{2} \rightarrow x_{1} x_{2},
\end{gathered}
$$

$\langle 43\rangle$,

where $\zeta\left(x_{2}, x_{3}\right)$ is $\varphi\left(x_{2}, x_{3}\right) x_{2} v\left(x_{2}, x_{3}\right)$; and

$$
\begin{gathered}
x_{1} \rightarrow \varphi\left(x_{2}, x_{3}\right) x_{1}, \\
x_{3} \rightarrow x_{1} x_{3},
\end{gathered}
$$

$\langle 44\rangle$,

where $\zeta\left(x_{2}, x_{3}\right)$ is $\varphi\left(x_{2}, x_{3}\right) x_{3} v\left(x_{2}, x_{3}\right)$.

Proposition 46. The general solution of the equation

46

$$
x_{1} x_{2} x_{3} x_{4}=\zeta\left(x_{1}, x_{2}\right) x_{3},
$$

where $x_{1}, x_{2}$ occur in $\zeta\left(x_{1}, x_{2}\right)$, is described by the transformation

$$
\left\{\begin{array}{l}
x_{1} \rightarrow x_{1}^{\alpha}, \\
x_{2} \rightarrow x_{1}^{\beta},
\end{array}\right.
$$

Proposition 47. The general solution of the equation

47

$$
x_{1} x_{2} x_{3} x_{4}=\zeta\left(x_{1}, x 2\right) x_{5},
$$

where $x_{1}, x_{2}$ occur in $\zeta\left(x_{1}, x_{2}\right)$, is described by the transformation

$$
\left\{\begin{array}{l}
x_{1} \rightarrow x_{1}^{\alpha}, \\
x_{2} \rightarrow x_{1}^{\beta},
\end{array}\right.
$$

$\langle 33\rangle$.

Proposition 48. The general solution of the equation 48

$$
x_{2} x_{1} x_{3} x_{4}=\left(x_{3} x_{1}\right)^{r+1} \zeta\left(x_{1} x_{3},\left(x_{1} x_{3}\right)^{\lambda} x_{1}\right) x_{2}
$$

with $\partial\left(x_{2}\right) \leq \partial\left(x_{3} x_{1}\right)$ is described by the transformations

$$
\begin{gathered}
x_{3} \rightarrow x_{2} x_{3}, \\
x_{4} \rightarrow\left(x_{3} x_{1} x_{2}\right)^{r}, \\
\langle\mathbf{2}\rangle,
\end{gathered}
$$

where $\zeta\left(x_{1} x_{3},\left(x_{1} x_{3}\right)^{\lambda} x_{1}\right)$ is 1 ;

$$
\begin{gathered}
x_{3} \rightarrow x_{2} x_{3}, \\
x_{4} \rightarrow x_{4} P, \\
\langle\mathbf{1 2}\rangle,
\end{gathered}
$$


where $\zeta\left(x_{1} x_{2} x_{3},\left(x_{1} x_{2} x_{3}\right)^{\lambda} x_{1}\right) x_{2}$ can be $x_{1} x_{2} P$;

$$
\begin{aligned}
x_{3} & \rightarrow x_{2} x_{3}, \\
x_{4} & \rightarrow x_{4} P, \\
& \langle\mathbf{2 0}\rangle,
\end{aligned}
$$

where $\zeta\left(x_{1} x_{2} x_{3},\left(x_{1} x_{2} x_{3}\right)^{\lambda} x_{1}\right) x_{2}$ can be $x_{1}^{q+2} x_{2} P$;

$$
\begin{gathered}
x_{2} \rightarrow x_{3} x_{2}, \\
x_{1} \rightarrow x_{2} x_{1}, \\
x_{4} \rightarrow\left(x_{1} x_{3} x_{2}\right)^{r},
\end{gathered}
$$$$
\langle\mathbf{2}\rangle,
$$

where $\zeta\left(x_{1} x_{3},\left(x_{1} x_{3}\right)^{\lambda} x_{1}\right)$ is 1 ;

$$
\begin{gathered}
x_{2} \rightarrow x_{3} x_{2}, \\
x_{1} \rightarrow x_{2} x_{1}, \\
x_{4} \rightarrow x_{1}\left(x_{3} x_{2} x_{1}\right)^{r-1} \zeta\left(x_{2} x_{1} x_{3},\left(x_{2} x_{1} x_{3}\right)^{\lambda} x_{2} x_{1}\right) x_{3} x_{2},
\end{gathered}
$$

where $r>0$; and

$$
\begin{gathered}
x_{2} \rightarrow x_{3} x_{2}, \\
x_{1} \rightarrow x_{2} x_{1}, \\
x_{4} \rightarrow x_{4} P, \\
\left\{\begin{array}{l}
x_{1} \rightarrow x_{1}^{\alpha}, \\
x_{2} \rightarrow x_{1}^{\beta},
\end{array}\right.
\end{gathered}
$$

where $r=0$, and $x_{1} \zeta\left(x_{2} x_{1} x_{3},\left(x_{2} x_{1} x_{3}\right)^{\lambda} x_{2} x_{1}\right) x_{3} x_{2}$ can be $\left(x_{2} x_{1}\right)^{s+1} x_{3} P$.

Proposition 49. The general solution of the equation

49

$$
x_{2} x_{1} x_{3} x_{4}=x_{1} x_{3} \zeta\left(x_{1} x_{3},\left(x_{1} x_{3}\right)^{\lambda} x_{1}\right) x_{2}
$$

with $\partial\left(x_{2}\right) \leq \partial\left(x_{1} x_{3}\right)$ is described by the transformations

$$
\begin{gathered}
x_{1} \rightarrow x_{2} x_{1}, \\
x_{4} \rightarrow P,
\end{gathered}
$$

where $\zeta\left(x_{2} x_{1} x_{3},\left(x_{2} x_{1} x_{3}\right)^{\lambda} x_{2} x_{1}\right) x_{2}$ can be $x_{2} P$;

$$
\begin{gathered}
x_{2} \rightarrow x_{1} x_{2}, \\
x_{3} \rightarrow x_{2} x_{3}, \\
x_{4} \rightarrow P,
\end{gathered}
$$

$\langle\mathbf{2}\rangle$,

where $\zeta\left(x_{1} x_{2} x_{3},\left(x_{1} x_{2} x_{3}\right)^{\lambda} x_{1}\right) x_{1} x_{2}$ can be $x_{1} x_{2} P$;

$$
\begin{aligned}
& x_{2} \rightarrow x_{1} x_{2}, \\
& x_{3} \rightarrow x_{2} x_{3}, \\
& x_{4} \rightarrow x_{4} P,
\end{aligned}
$$

$\langle\mathbf{1 2}\rangle$, 
where $\zeta\left(x_{1} x_{2} x_{3},\left(x_{1} x_{2} x_{3}\right)^{\lambda} x_{1}\right) x_{1} x_{2}$ can be $x_{1}^{2} x_{2} P$;

$$
\begin{aligned}
& x_{2} \rightarrow x_{1} x_{2}, \\
& x_{3} \rightarrow x_{2} x_{3}, \\
& x_{4} \rightarrow x_{4} P,
\end{aligned}
$$

$\langle\mathbf{2 0}\rangle$,

where $\zeta\left(x_{1} x_{2} x_{3},\left(x_{1} x_{2} x_{3}\right)^{\lambda} x_{1}\right) x_{1} x_{2}$ can be $x_{1}^{q+3} x_{2} P$; and

$$
\begin{aligned}
& x_{2} \rightarrow x_{1} x_{2}, \\
& x_{3} \rightarrow x_{2} x_{3}, \\
& x_{4} \rightarrow x_{4} P,
\end{aligned}
$$

$\langle\mathbf{2 9}\rangle$,

where $\zeta\left(x_{1} x_{2} x_{3},\left(x_{1} x_{2} x_{3}\right)^{\lambda} x_{1}\right) x_{1} x_{2}$ is $x_{2} x_{3} x_{1} P$.

Proposition 50. The general solution of the equation

50

$$
x_{2} x_{1} x_{3} x_{4}=\left(x_{1} x_{3}\right)^{\lambda} x_{1} \zeta\left(x_{1} x_{3}, x_{3} x_{1},\left(x_{1} x_{3}\right)^{\lambda} x_{1}\right) x_{2}
$$

with $\partial\left(x_{2}\right) \leq \partial\left(\left(x_{1} x_{3}\right)^{\lambda} x_{1}\right)$ is described by the transformations

$$
\begin{gathered}
x_{2} \rightarrow\left(x_{1} x_{3}\right)^{\tau} x_{2}, \\
x_{1} \rightarrow x_{2} x_{1}, \\
x_{4} \rightarrow P,
\end{gathered}
$$

$\langle\mathbf{2}\rangle$,

where $\left(x_{2} x_{1} x_{3}\right)^{\lambda-\tau} \zeta\left(x_{2} x_{1} x_{3}, x_{3} x_{2} x_{1},\left(x_{2} x_{1} x_{3}\right)^{\lambda} x_{2} x_{1}\right)\left(x_{2} x_{1} x_{3}\right)^{\tau} x_{2}$ can be $x_{3} x_{2} P$;

$$
\begin{gathered}
x_{2} \rightarrow\left(x_{1} x_{3}\right)^{\tau} x_{2}, \\
x_{1} \rightarrow x_{2} x_{1}, \\
x_{3} \rightarrow 1, \\
x_{4} \rightarrow 1,
\end{gathered}
$$

$\langle\mathbf{1}\rangle$,

where $\left(x_{2} x_{1} x_{3}\right)^{\lambda-\tau} \zeta\left(x_{2} x_{1} x_{3}, x_{3} x_{2} x_{1},\left(x_{2} x_{1} x_{3}\right)^{\lambda} x_{2} x_{1}\right)\left(x_{2} x_{1} x_{3}\right)^{\tau} x_{2}$ can be $x_{2}$;

$$
\begin{gathered}
x_{2} \rightarrow\left(x_{1} x_{3}\right)^{\tau} x_{2}, \\
x_{1} \rightarrow x_{2} x_{1}, \\
x_{4} \rightarrow x_{4} P, \\
\left\{\begin{array}{l}
x_{1} \rightarrow x_{1}^{\alpha}, \\
x_{2} \rightarrow x_{1}^{\beta},
\end{array}\right.
\end{gathered}
$$

where $\left(x_{2} x_{1} x_{3}\right)^{\lambda-\tau} \zeta\left(x_{2} x_{1} x_{3}, x_{3} x_{2} x_{1},\left(x_{2} x_{1} x_{3}\right)^{\lambda} x_{2} x_{1}\right)\left(x_{2} x_{1} x_{3}\right)^{\tau} x_{2}$ can be $x_{2} x_{1} x_{3} P$;

$$
\begin{gathered}
x_{2} \rightarrow\left(x_{1} x_{3}\right)^{\tau} x_{1} x_{2}, \\
x_{3} \rightarrow x_{2} x_{3}, \\
x_{4} \rightarrow P,
\end{gathered}
$$

$\langle\mathbf{2}\rangle$,

where $\left(x_{2} x_{3} x_{1}\right)^{\lambda-\tau-1} \zeta\left(x_{1} x_{2} x_{3}, x_{2} x_{3} x_{1},\left(x_{1} x_{2} x_{3}\right)^{\lambda} x_{1}\right)\left(x_{1} x_{2} x_{3}\right)^{\tau} x_{1} x_{2}$ is $x_{1} x_{2} P$; and

$$
\begin{gathered}
x_{2} \rightarrow\left(x_{1} x_{3}\right)^{\tau} x_{1} x_{2}, \\
x_{3} \rightarrow x_{2} x_{3}, \\
x_{4} \rightarrow x_{4} P,
\end{gathered}
$$

$\langle\mathbf{2 9}\rangle$, 
where $\left(x_{2} x_{3} x_{1}\right)^{\lambda-\tau-1} \zeta\left(x_{1} x_{2} x_{3}, x_{2} x_{3} x_{1},\left(x_{1} x_{2} x_{3}\right)^{\lambda} x_{1}\right)\left(x_{1} x_{2} x_{3}\right)^{\tau} x_{1} x_{2}=x_{2} x_{3} x_{1} P$.

Proposition 51. The general solution of the equation

51

$$
x_{2} x_{1} x_{3} x_{4}=\left(x_{3} x_{1}\right)^{r+1} \zeta\left(x_{2} x_{3},\left(x_{1} x_{3}\right)^{\lambda} x_{1}\right) x_{5}
$$

with $\partial\left(x_{2}\right) \leq \partial\left(x_{3} x_{1}\right)$ is described by the transformations

$$
\begin{aligned}
& x_{3} \rightarrow x_{2} x_{3}, \\
& x_{5} \rightarrow x_{5} x_{4},
\end{aligned}
$$

$\langle\mathbf{1 0}\rangle$,

where $r=0$ and $\zeta\left(x_{1} x_{3},\left(x_{1} x_{3}\right)^{\lambda} x_{1}\right)$ is 1 ;

$$
\begin{gathered}
x_{3} \rightarrow x_{2} x_{3}, \\
x_{4} \rightarrow P,
\end{gathered}
$$

$\langle\mathbf{2}\rangle$

where $\left(x_{2} x_{3} x_{1}\right)^{r} \zeta\left(x_{1} x_{2} x_{3},\left(x_{1} x_{2} x_{3}\right)^{\lambda} x_{1}\right) x_{5}$ is $x_{2} P$;

$$
\begin{aligned}
x_{3} & \rightarrow x_{2} x_{3}, \\
x_{4} & \rightarrow x_{4} P,
\end{aligned}
$$

$\langle\mathbf{1 2}\rangle$,

where $\left(x_{2} x_{3} x_{1}\right)^{r} \zeta\left(x_{1} x_{2} x_{3},\left(x_{1} x_{2} x_{3}\right)^{\lambda} x_{1}\right) x_{5}$ can be $x_{1} x_{2} P$;

$$
\begin{aligned}
x_{3} & \rightarrow x_{2} x_{3}, \\
x_{4} & \rightarrow x_{4} P,
\end{aligned}
$$

$\langle\mathbf{2 0}\rangle$,

where $\left(x_{2} x_{3} x_{1}\right)^{r} \zeta\left(x_{1} x_{2} x_{3},\left(x_{1} x_{2} x_{3}\right)^{\lambda} x_{1}\right) x_{5}$ can be $x_{1}^{q+2} x_{2} P$;

$$
\begin{gathered}
x_{2} \rightarrow x_{3} x_{2}, \\
x_{1} \rightarrow x_{2} x_{1}, \\
x_{5} \rightarrow x_{5} x_{3} x_{4},
\end{gathered}
$$

$\langle 4\rangle$,

where $r=0, \zeta\left(x_{1} x_{3},\left(x_{1} x_{3}\right)^{\lambda} x_{1}\right)=1$;

$$
\begin{gathered}
x_{2} \rightarrow x_{3} x_{2}, \\
x_{1} \rightarrow x_{2} x_{1}, \\
x_{4} \rightarrow x_{1}\left(x_{3} x_{2} x_{1}\right)^{r-1} \zeta\left(x_{2} x_{1} x_{3},\left(x_{2} x_{1} x_{3}\right)^{\lambda} x_{2} x_{1}\right) x-5, \\
\langle\mathbf{2}\rangle,
\end{gathered}
$$

where $r>0$;

$$
\begin{gathered}
x_{2} \rightarrow x_{3} x_{2}, \\
x_{1} \rightarrow x_{2} x_{1}, \\
x_{4} \rightarrow x_{4} P, \\
\left\{\begin{array}{l}
x_{1} \rightarrow x_{1}^{\alpha}, \\
x_{2} \rightarrow x_{1}^{\beta},
\end{array}\right.
\end{gathered}
$$

$\langle\mathbf{5}\rangle$, 
where $r=0$ and $\zeta\left(x_{2} x_{1} x_{3},\left(x_{2} x_{1} x_{3}\right)^{\lambda} x_{2} x_{1}\right) x_{5}$ can be $\left(x_{2} x_{1}\right)^{q+1} x_{3} P$; and

$$
\begin{gathered}
x_{2} \rightarrow x_{3} x_{2}, \\
x_{1} \rightarrow x_{2} x_{1}, \\
\left\{\begin{array}{l}
x_{1} \rightarrow x_{1}^{\alpha}, \\
x_{2} \rightarrow x_{1}^{\beta},
\end{array}\right.
\end{gathered}
$$

$\langle 33\rangle$,

where $r=0$ and $\zeta\left(x_{2} x_{1} x_{3},\left(x_{2} x_{1} x_{3}\right)^{\lambda} x_{2} x_{1}\right)$ can be $\left(x_{2} x_{1}\right)^{q+1}$.

Proposition 52. The general solution of the equation

52

$$
x_{2} x_{1} x_{3} x_{4}=x_{1} x_{3} \zeta\left(x_{1} x_{3},\left(x_{1} x_{3}\right)^{\lambda} x_{1}\right) x_{5}
$$

with $\partial\left(x_{2}\right) \leq \partial\left(x_{1} x_{3}\right)$ is described by the transformations

$$
\begin{aligned}
& x_{1} \rightarrow x_{2} x_{1}, \\
& x_{5} \rightarrow x_{5} x_{4},
\end{aligned}
$$

$\langle 7\rangle$,

where $\zeta\left(x_{1} x_{3},\left(x_{1} x_{3}\right)^{\lambda} x_{1}\right)$ is 1 ;

$$
\begin{aligned}
& x_{1} \rightarrow x_{2} x_{1}, \\
& x_{4} \rightarrow P x_{5},
\end{aligned}
$$

$\langle\mathbf{2}\rangle$,

where $\zeta\left(x_{2} x_{1} x_{3},\left(x_{2} x_{1} x_{3}\right)^{\lambda} x_{2} x_{1}\right)$ can be $x_{2} P$;

$$
\begin{aligned}
& x_{2} \rightarrow x_{1} x_{2}, \\
& x_{3} \rightarrow x_{2} x_{3}, \\
& x_{5} \rightarrow x_{5} x_{4},
\end{aligned}
$$

$\langle 6\rangle$,

where $\zeta\left(x_{1} x_{3},\left(x_{1} x_{3}\right)^{\lambda} x_{1}\right)$ is 1 ;

$$
\begin{aligned}
& x_{2} \rightarrow x_{1} x_{2}, \\
& x_{3} \rightarrow x_{2} x_{3},
\end{aligned}
$$

$\langle 39\rangle$,

where $\zeta\left(x_{1} x_{2} x_{3},\left(x_{1} x_{2} x_{3}\right)^{\lambda} x_{1}\right)$ can be $x_{1}^{q+1}$;

$$
\begin{gathered}
x_{2} \rightarrow x_{1} x_{2}, \\
x_{3} \rightarrow x_{2} x_{3}, \\
x_{4} \rightarrow P
\end{gathered}
$$

$\langle\mathbf{2}\rangle$,

where $\zeta\left(x_{1} x_{2} x_{3},\left(x_{1} x_{2} x_{3}\right)^{\lambda} x_{1}\right)$ can be $x_{1} x_{2} P$;

$$
\begin{aligned}
& x_{2} \rightarrow x_{1} x_{2}, \\
& x_{3} \rightarrow x_{2} x_{3}, \\
& x_{4} \rightarrow x_{4} P,
\end{aligned}
$$

$\langle\mathbf{1 2}\rangle$,

where $\zeta\left(x_{1} x_{2} x_{3},\left(x_{1} x_{2} x_{3}\right)^{\lambda} x_{1}\right)$ can be $x_{1}^{2} x_{2} P$; and

$$
\begin{aligned}
& x_{2} \rightarrow x_{1} x_{2}, \\
& x_{3} \rightarrow x_{2} x_{3}, \\
& x_{4} \rightarrow x_{4} P,
\end{aligned}
$$

$\langle\mathbf{2 0}\rangle$, 
where $\zeta\left(x_{1} x_{2} x_{3},\left(x_{1} x_{2} x_{3}\right)^{\lambda} x_{1}\right)$ can be $x_{1}^{s+3} x_{2} P$.

Proposition 53. The general solution of the equation

53

$$
x_{2} x_{1} x_{3} x_{4}=\left(x_{1} x_{3}\right)^{\lambda} x_{1} \zeta\left(x_{1} x_{3},\left(x_{1} x_{3}\right)^{\lambda} x_{1}\right) x_{5}
$$

with $\partial\left(x_{2}\right) \leq \partial\left(\left(x_{1} x_{3}\right)^{\lambda} x_{1}\right)$ is described by the transformations

$$
\begin{gathered}
x_{2} \rightarrow\left(x_{1} x_{3}\right)^{\lambda} x_{2}, \\
x_{1} \rightarrow x_{2} x_{1}, \\
x_{5} \rightarrow x_{5} x_{3} x_{4},
\end{gathered}
$$

$\langle 4\rangle$,

where $\zeta\left(x_{1} x_{3},\left(x_{1} x_{3}\right)^{\lambda} x_{1}\right)$ is 1 ;

$$
\begin{gathered}
x_{2} \rightarrow\left(x_{1} x_{3}\right)^{\tau} x_{2}, \\
x_{1} \rightarrow x_{2} x_{1}, \\
x_{4} \rightarrow x_{4} P, \\
\left\{\begin{array}{l}
x_{1} \rightarrow x_{1}^{\alpha}, \\
x_{2} \rightarrow x_{1}^{\beta},
\end{array}\right.
\end{gathered}
$$

$\langle\mathbf{5}\rangle$,

where $\left(x_{2} x_{1} x_{3}\right)^{\lambda-\tau} \zeta\left(x_{2} x_{1} x_{3},\left(x_{2} x_{1} x_{3}\right)^{\lambda} x_{2} x_{1}\right) x_{5}$ can be $\left(x_{2} x_{1}\right)^{q+1} x_{3} P$;

$$
\begin{gathered}
x_{2} \rightarrow\left(x_{1} x_{3}\right)^{\tau} x_{2}, \\
x_{1} \rightarrow x_{2} x_{1}, \\
\left\{\begin{array}{l}
x_{1} \rightarrow x_{1}^{\alpha}, \\
x_{2} \rightarrow x_{1}^{\beta},
\end{array}\right.
\end{gathered}
$$

$\langle\mathbf{3 3}\rangle$,

where $\left(x_{2} x_{1} x_{3}\right)^{\lambda-\tau} \zeta\left(x_{2} x_{1} x_{3},\left(x_{2} x_{1} x_{3}\right)^{\lambda} x_{2} x_{1}\right)$ can be $\left(x_{2} x_{1}\right)^{q+1}$;

$$
\begin{aligned}
x_{2} \rightarrow & \left(x_{1} x_{3}\right)^{\lambda-1} x_{1} x_{2}, \\
& x_{3} \rightarrow x_{2} x_{3}, \\
& x_{5} \rightarrow x_{5} x_{4},
\end{aligned}
$$

$\langle\mathbf{1 0}\rangle$,

where $\zeta\left(x_{1} x_{3},\left(x_{1} x_{3}^{\lambda} x_{1}\right)\right.$ is 1 ;

$$
\begin{gathered}
x_{2} \rightarrow\left(x_{1} x_{3}\right)^{\lambda-1} x_{1} x_{2}, \\
x_{3} \rightarrow x_{2} x_{3},
\end{gathered}
$$

$\langle\mathbf{3 9}\rangle$,

where $\zeta\left(x_{1} x_{2} x_{3},\left(x_{1} x_{2} x_{3}\right)^{\lambda} x_{1}\right)$ can be $x_{1}^{s+1}$;

$$
\begin{gathered}
x_{2} \rightarrow\left(x_{1} x_{3}\right)^{\lambda-1}, \\
x_{3} \rightarrow x_{2} x_{3}, \\
x_{4} \rightarrow P,
\end{gathered}
$$

$\langle\mathbf{2}\rangle$,

where $\zeta\left(x_{1} x_{2} x_{3},\left(x_{1} x_{2} x_{3}\right)^{\lambda} x_{1}\right)$ can be $x_{1} x_{2} P$;

$$
\begin{gathered}
x_{2} \rightarrow\left(x_{1} x_{3}\right)^{\lambda-1}, \\
x_{3} \rightarrow x_{2} x_{3}, \\
x_{4} \rightarrow x_{4} P,
\end{gathered}
$$

$\langle\mathbf{1 2}\rangle$, 
where $\zeta\left(x_{1} x_{2} x_{3},\left(x_{1} x_{2} x_{3}\right)^{\lambda} x_{1}\right)$ can be $x_{1}^{2} x_{2} P$; and

$$
\begin{gathered}
x_{2} \rightarrow\left(x_{1} x_{3}\right)^{\lambda-1}, \\
x_{3} \rightarrow x_{2} x_{3}, \\
x_{4} \rightarrow x_{4} P,
\end{gathered}
$$

$\langle\mathbf{2 0}\rangle$,

where $\zeta\left(x_{1} x_{2} x_{3},\left(x_{1} x_{2} x_{3}\right)^{\lambda} x_{1}\right)$ can be $x_{1}^{s+3} x_{2}$.

Proposition 54. The general solution of the equation

54

$$
x_{1} x_{2} x_{3} x_{4}=x_{3}^{r+1} x_{1} \zeta\left(x_{1}, x_{3}\right) x_{2},
$$

where $r$ is a natural number, is described by the transformations

$$
\begin{aligned}
& \left\{\begin{array}{l}
x_{1} \rightarrow 1 \\
x_{3} \rightarrow 1 \\
x_{4} \rightarrow 1
\end{array}\right. \\
& \left\{\begin{array}{l}
x_{1} \rightarrow\left(x_{1} x_{3}\right)^{\lambda} x_{1}, \\
x_{3} \rightarrow x_{1} x_{3} \\
x_{2} \rightarrow\left(\left(x_{3} x_{1}\right)^{r+1} \zeta\left(\left(x_{1} x_{3}\right)^{\lambda} x_{1}, x_{1} x_{3}\right)\right)^{\alpha} v\left(\left(x_{1} x_{3}\right)^{\lambda} x_{1}, x_{3} x_{1}, x_{1} x_{3}\right) x_{2},
\end{array}\right.
\end{aligned}
$$

where $x_{5}^{r+1} \zeta\left(x_{1}, x_{3}\right)$ is $v\left(x_{1}, x_{5}, x_{3}\right) \varphi\left(x_{1}, x_{5}, x_{3}\right)$, followed by one of the three transformations $\langle\mathbf{4 8}\rangle,\langle\mathbf{4 9}\rangle,\langle\mathbf{5 0}\rangle$.

Proposition 55. The general solution of the equation

55

$$
x_{1} x_{2} x_{3} x_{4}=x_{3}^{r+1} x_{1} \zeta\left(x_{1}, x_{3}\right) x_{5},
$$

where $r$ is a natural number, is described by the transformations

$$
\left\{\begin{array}{l}
x_{1} \rightarrow\left(x_{1} x_{3}\right)^{\lambda} x_{1} \\
x_{3} \rightarrow x_{1} x_{3} \\
x_{2} \rightarrow \varphi\left(\left(x_{1} x_{3}\right)^{\lambda} x_{1}, x_{3} x_{1}, x_{1} x_{3}\right) x_{2},
\end{array}\right.
$$

where $x_{6}^{r+1} \zeta\left(x_{1}, x_{3}\right)$ is $\varphi\left(x_{1}, x_{6}, x_{3}\right) v\left(x_{1}, x_{6}, x_{3}\right)$, followed by one of the four transformations $\langle\mathbf{5 1}\rangle,\langle\mathbf{5 2}\rangle,\langle\mathbf{5 3}\rangle$, or $x_{5} \rightarrow x_{2} x_{1} x_{3} x_{4}$, where $v\left(x_{1}, x_{6}, x_{3}\right)$ is 1 .

Proposition 56. The general solution of the equation

56

$$
x_{1} x_{2} x_{3} x_{4}=\zeta\left(x_{1}, x_{2}, x_{3}\right) x_{5}
$$

is described by the transformations $\langle\mathbf{4 2}\rangle ;\langle\mathbf{4 6}\rangle ;\langle\mathbf{5 4}\rangle ;\langle\mathbf{4 5}\rangle ;\langle\mathbf{4 7}\rangle ;\langle\mathbf{5 5}\rangle ;\langle\mathbf{3 4}\rangle ;\langle\mathbf{3 6}\rangle$; $x_{5} \rightarrow x_{1} x_{2} x_{3} x_{4}$, where $\zeta\left(x_{1}, x_{2}, x_{3}\right)=1$.

Proof. If $x_{1}, x_{2}, x_{3}$ occur in $\zeta\left(x_{1}, x_{2}, x_{3}\right)$, then the equation $\mathbf{5 6}$ has one of the following three forms:

$$
E_{1}: \quad x_{1} x_{2} x_{3} x_{4}=v\left(x_{2}, x_{3}\right) x_{1} \varphi\left(x_{1}, x_{2}, x_{3}\right) x_{5},
$$

where $x_{2}$ and $x_{3}$ occur in $v\left(x_{2}, x_{3}\right)$;

$$
E_{2}: \quad x_{1} x_{2} x_{3} x_{4}=v\left(x_{1}, x_{2}\right) x_{3} \varphi\left(x_{1}, x_{2}, x_{3}\right) x_{5},
$$

where $x_{1}$ and $x_{2}$ occur in $v\left(x_{1}, x_{2}\right)$;

$$
E_{3}: \quad x_{1} x_{2} x_{3} x_{4}=v\left(x_{1}, x_{3}\right) x_{2} \varphi\left(x_{1}, x_{2}, x_{3}\right) x_{5},
$$

where $x_{1}$ and $x_{3}$ occur in $v\left(x_{1}, x_{3}\right)$. 
The equation $E_{1}$ can be reduced by the transformation

$$
x_{4} \rightarrow x_{4} \varphi\left(x_{1}, x_{2}, x_{3}\right) x_{5}
$$

to the equation $\mathbf{4 2}$.

The equation $E_{2}$ can be reduced by the transformation

$$
x_{4} \rightarrow x_{4} \varphi\left(x_{1}, x_{2}, x_{3}\right) x_{5}
$$

to the equation 46.

The equation $E_{3}$ can be reduced by the transformation

$$
x_{4} \rightarrow x_{4} \varphi\left(x_{1}, x_{2}, x_{3}\right) x_{5}
$$

to the equation $\mathbf{5 4}$.

If only $x_{2}, x_{3}$ occur in $\zeta\left(x_{1}, x_{2}, x_{3}\right)$, then $\mathbf{5 6}$ is $\mathbf{4 5}$.

If only $x_{1}, x_{2}$ occur in $\zeta\left(x_{1}, x_{2}, x_{3}\right)$, then $\mathbf{5 6}$ is $\mathbf{4 7}$.

If only $x_{1}, x_{3}$ occur in $\zeta\left(x_{1}, x_{2}, x_{3}\right)$, then $\mathbf{5 6}$ is $\mathbf{5 5}$.

If only $x_{2}$ occurs in $\zeta\left(x_{1}, x_{2}, x_{3}\right)$, then $\mathbf{5 6}$ is $\mathbf{3 4}$.

If only $x_{3}$ occurs in $\zeta\left(x_{1}, x_{2}, x_{3}\right)$, then $\mathbf{5 6}$ is $\mathbf{3 6}$.

If $\zeta\left(x_{1}, x_{2}, x_{3}\right)$ is the empty word, then $\mathbf{5 6}$ has the form $x_{1} x_{2} x_{3} x_{4}=x_{5}$.

\section{REFERENCES}

[1] R. C. Lyndon, Equations in free groups, Trans. Amer. Math. Soc. 96 (1960), 445-457. MR 27:1488

[2] Groups with parametric exponents, Trans. Amer. Math. Soc. 96 (1960), 518-533. MR 27:1487

[3] Yu. I. Khmelevskiǔ, Solution of word equations in three unknowns, Dokl. Akad. Nauk SSSR 177 (1967), 1023-1025; English transl., Soviet Math. Dokl. 8 (1967), 1554-1556. MR 36:3899

[4] _ Equation in free semigroups, Trudy Mat. Inst. Steklov. 107 (1971); English transl., Proc. Steklov Inst. Math. 107 (1971) (1976). MR 51:5808; MR 52:14094

[5] G. S. Makanin and P. Goralcik, Structure of the solutions of one parametric equation, Report of research, LIR, INSA of Rouen, April 1996.

[6] G. S. Makanin and H. Abdulrab, Functions for the general solution of parametric word equations, Report of research, LIR, INSA of Rouen, June 1996.

[7] G. S. Makanin, Systems of standard equations in words of an n-layer alphabet with unknowns, Sibirsk. Mat. Zh. 19 (1978), 637-645; English transl., Siberian Math. J. 19 (1978), 448-454. MR 80a:20039

[8] _ Recognition of the rank of equations in a free semigroup, Izv. Akad. Nauk SSSR Ser. Mat. 43 (1979), 547-602; English transl., Math. USSR Izv. 14 (1980), 499-545. MR 80h:20084

[9] M. Lothaire, Combinatorics on words, Addison-Wesley, 1983. MR 84g:05002

Steklov Mathematical Institute, Vavilova 42, 117 966, Moscow GSP-1, Russia 